\title{
Genetic architecture of collective behaviors in zebrafish
}

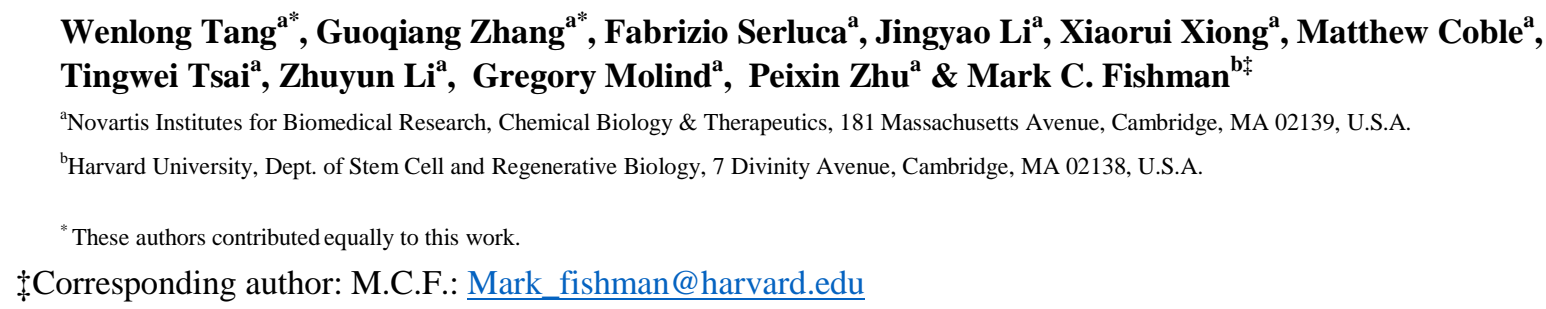

Abstract Collective behaviors of groups of animals, such as schooling and shoaling of fish, are central to species survival, but genes that regulate these activities are not known. Here we parsed collective behavior of groups of adult zebrafish using computer vision and unsupervised machine learning into a set of highly reproducible, unitary, several hundred millisecond states and transitions, which together can account for the entirety of relative positions and postures of groups of fish. Using CRISPR-Cas9 we then targeted for knockout 35 genes associated with autism and schizophrenia. We found mutations in three genes had distinctive effects on the amount of time spent in the specific states or transitions between states. Mutation in immp2l (inner mitochondrial membrane peptidase 2like gene) enhances states of cohesion, so increases shoaling; mutation in in the Nav1.1 sodium channel, scn1lab+/- causes the fish to remain scattered without evident social interaction; and mutation in the adrenergic receptor, adra1aa-/-, keeps fish close together and retards transitions between states, leaving fish motionless for long periods. Motor and visual functions seemed relatively wellpreserved. This work shows that the behaviors of fish engaged in collective activities are built from a set of stereotypical states. Single gene mutations can alter propensities to collective actions by changing the proportion of time spent in these states or the tendency to transition between states. This provides an approach to begin dissection of the molecular pathways used to generate and guide collective actions of groups of animals.

\section{Introduction}

Darwin recognized that survival depends not only upon the attributes of individuals but also upon coordination of larger groups, such as schools of fish, flocks of birds, and armies of ants (Darwin, 1871). The benefits of collective movement include improved predator avoidance (Ioannou, Guttal, \& Couzin, 2012; Ioannou, Morrell, Ruxton, \& Krause, 2009), foraging success (Krause, Hartmann, \& Pritchard, 1999; Torney, Berdahl, \& Couzin, 2011), and reduction in energy consumption due to favorable local currents for fish and birds (Krause, 
1994; Spedding, 2011). It is not known whether and how genetic changes in individuals affect group dynamics.

The schooling, shoaling, and other collective dynamics of fish have been beautifully described (Jolles, Boogert, Sridhar, Couzin, \& Manica; Katz, Tunstrom, Ioannou, Huepe, \& Couzin, 2011; Tunstrom et al., 2013). It appears that the sometimes rapid changes in direction and mode result from propagation of local interactions (Katz et al., 2011). The dynamics can be mathematically modelled as self-propelled particles subjected to forces such as attraction, repulsion, and alignment (Katz et al., 2011; Lukeman, Li, \& Edelstein-Keshet, 2010; Sumpter, 2010). No genes regulating such forces have been identified, although QTL linkage analysis suggests that schooling at least is under genetic control (Greenwood, Wark, Yoshida, \& Peichel, 2013).

Machine learning has begun to make more tractable the studies of complex behaviors (Anderson \& Perona, 2014), such as C. elegans foraging (Greene, Brown, et al., 2016), hydra basal behaviors (Han, Taralova, Dupre, \& Yuste, 2018), Drosophila mating and aggression (Dankert, Wang, Hoopfer, Anderson, \& Perona, 2009; Kabra, Robie, Rivera-Alba, Branson, \& Branson, 2013; Kravitz \& Huber, 2003), and mouse social behaviors (Hong et al., 2015; Kabra et al., 2013; Wiltschko et al., 2015). Unsupervised machine learning approaches, in particular, have the power to capture unknown patterns with as few a priori assumptions as possible (Todd, Kain, \& de Bivort, 2017), and are widely used in pattern learning, (Feng et al., 2017; Todd et al., 2017) even in cases where data is dense and not readily separable (Chuang, Tzeng, Chen, Wu, \& Chen, 2006; Siew, 2013).

Here we have found that unsupervised machine learning can describe fairly completely the collective motion of groups of adult zebrafish as a set of 17 stereotypical states and transitions between states. (Although most studies of zebrafish behavior use larvae (Baier, 2000; Brockerhoff et al., 1995; Friedrich, Jacobson, \& Zhu, 2010; Granato et al., 1996; Muto et al., 2005; Portugues \& Engert, 2009; Rihel et al., 2010; Zhu et al., 2009), we had to use adult fish because we found robust social behaviors developed late.) We mutated by CRISPR-Cas9 35 genes linked to human disorders of social behavior, such as autism and schizophrenia, with the hope that these genes might play a conserved social behavioral role through evolution. Overall these mutations did not completely add or delete states. Three mutations in particular, however, changed overall usage of states or transitions, with dramatic and consistent effects upon the collective group dynamics. 


\section{Resullts}

82 We first defined patterns of wild-type behavior. We find that the collective activity 83 became more robust and the behavioral repertoire expanded as the animals grew 84 (Supplemental Figure 1), so it was essential for our studies to use adult fish, at 85 least 3 months of age. We tracked groups of adult fish by continuous video 86 monitoring over half hour assays in an environmentally sheltered tank. As shown 87 in the video attached to Figure 1 (Supplemental Video 1), wild-type fish tend to 88 swim continuously and dynamically in relatively tight groups.

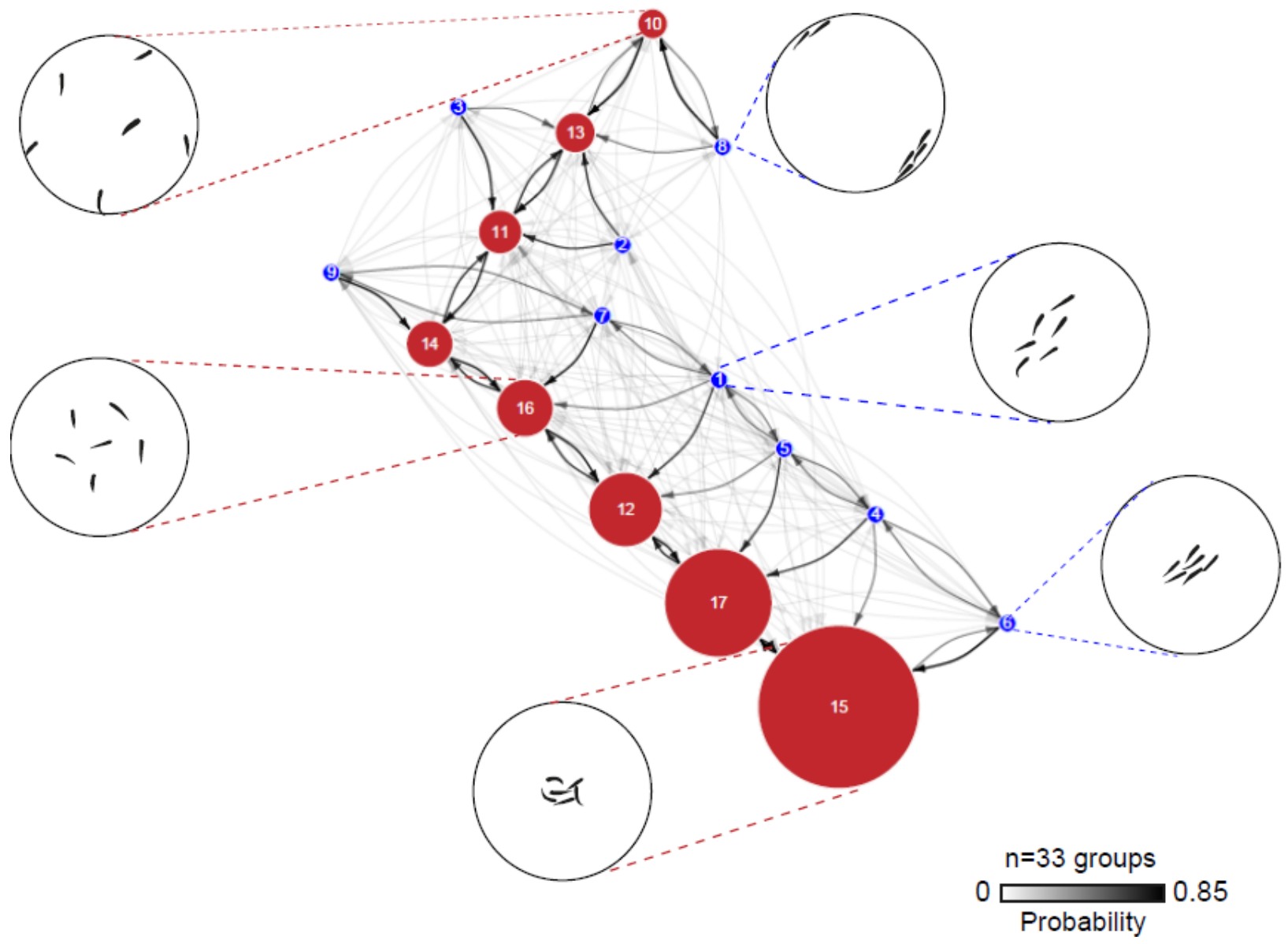

Figure 1. Ethogram of collective behavior in wild-type fish. Circle sizes 91 correspond to state usage ratios, and density of arrows to transition probabilities, 92 with polarized states in blue and unpolarized states in red ( $\mathrm{n}=33$ groups). Dendrogram of state usage is shown in Supplemental Figure 2. Schematic representations (not to scale) of selected states from both categories are shown as blow-ups. 
well as the probability of transition between states (Anderson \& Perona, 2014; Dankert et al., 2009). The ethogram of group behavior of wild-type fish is shown in Figure 1. Each circle represents a different state (with selected examples shown in blow-ups). For clarity, we have subdivided polarized (school) states (blue) from unpolarized (shoal) (red). The thickness of arrows shows transition probabilities between states. Certain states and transitions are used far more frequently than others (Supplemental Figures 2 and 3). The mean time spent in each state as a block is 0.78 seconds (Supplemental Figure 4). In this assay, fish spend far more time shoaling than schooling, and generally in more cohesive shoals, as shown by the prominence of states in the lower portion of the ethogram.

\section{Mutations}

For mutational analysis, we elected to mutate genes putatively related to autism or schizophrenia (Supplemental Table 1 and 2) because of the profound defects in social behavior that characterize these disorders, with the hope that their roles in social behaviors might be evolutionarily conserved, even if the behaviors themselves are species-specific. In order to provide sufficiently accurate information for design of effective CRISPR-Cas9 guide RNAs, we found it necessary to sequence and reassemble the complete genome of the AB strain (data available upon request).

We generated mutations in 35 genes (Supplemental file 1) and screened for changes to collective behavior in adult fish homozygous for the mutation (with the exception of scn1lab and slc18a2 which were not viable as homozygous animals so were evaluated as heterozygous animals). Behavior for each mutation was quantified on ethograms generated as above, and then all 35 compared to wild type AB. Using the Kullback-Leibler divergence approach (Kullback \& Leibler, 1951) we identified three mutations with particularly distinctive behavioral changes. Differences from wild-type were cross-validated by multi-class Support Vector Machines (SVM) (Supplemental Figure 5), and finally assessed by human expert validation using double blinded datasets. The ethograms for these three mutant fish are shown in Figure 2 and 3, along with exemplary videos. Some other mutations changed state usage in manner similar to the three described, but to a lesser degree (Supplemental Figure 6).

Enhanced cohesion Fish mutant in the inner mitochondrial membrane peptidase 2like gene, immp2l-/-, tend to aggregate tightly in small shoals, as shown in the video attached to Figure 2b (Supplemental Video 2). The increased shoaling is reflected in enhancement of two tight cohesion states in the lower part of the 
a wild-type
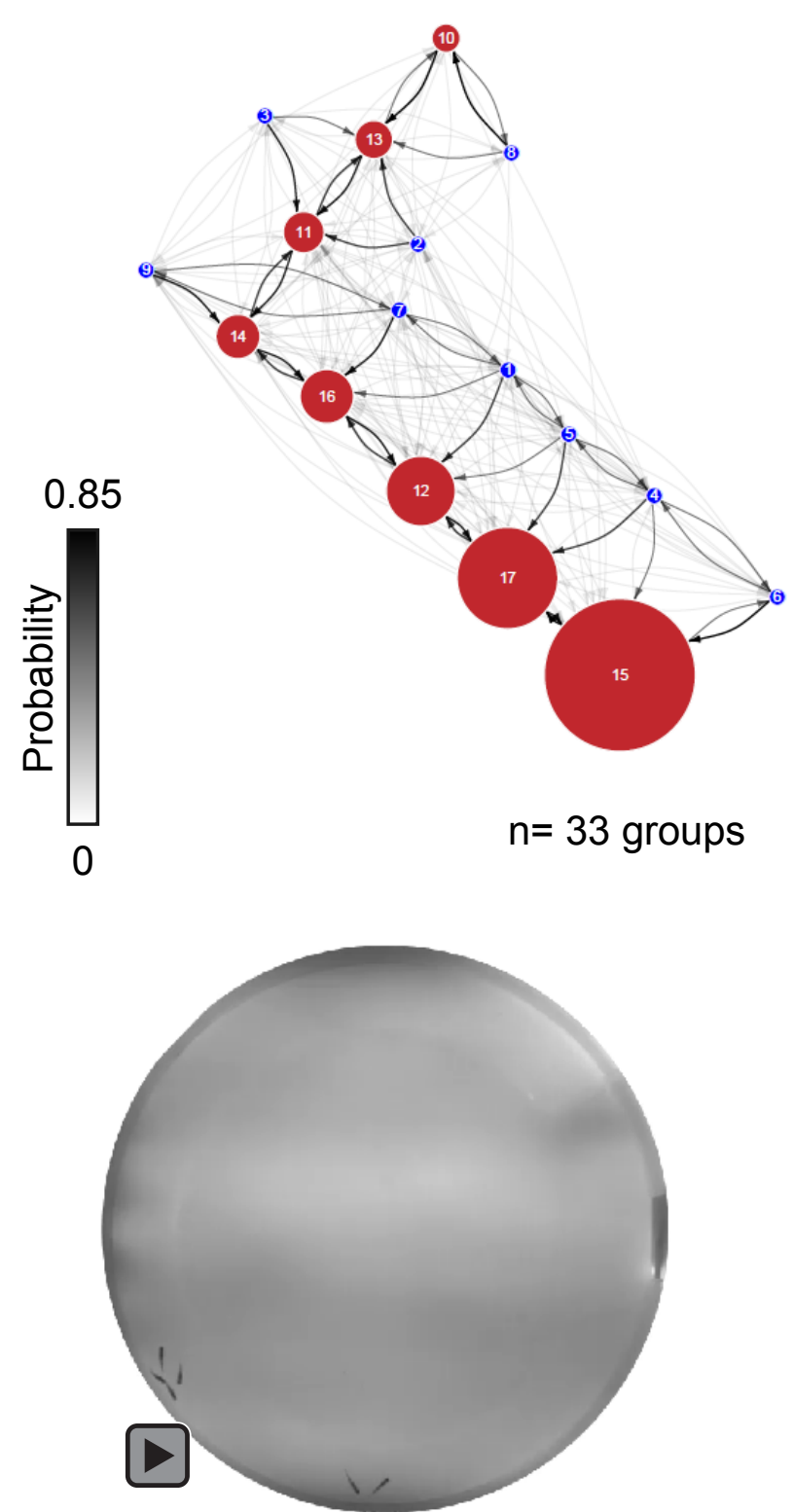

b immp2l-/-
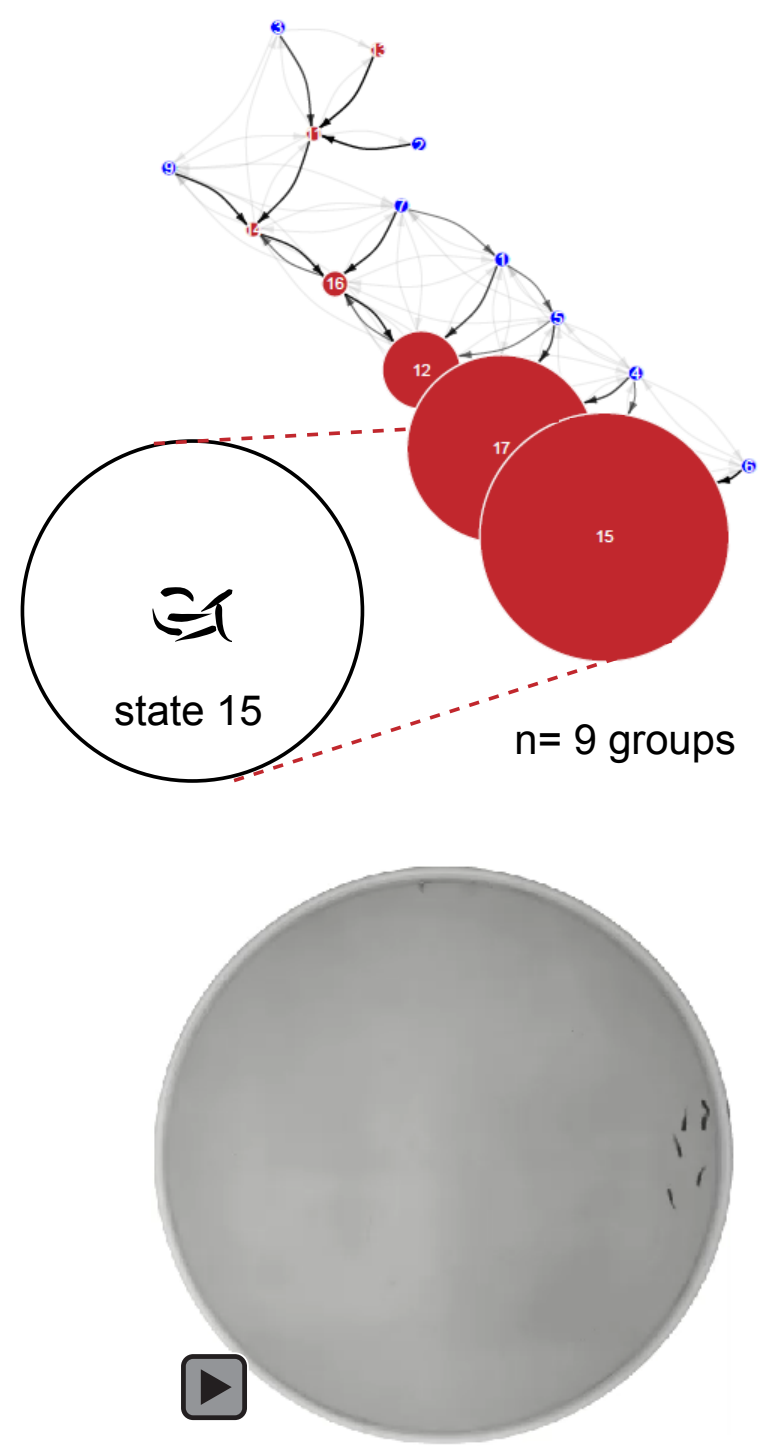

c scn1lab+/-

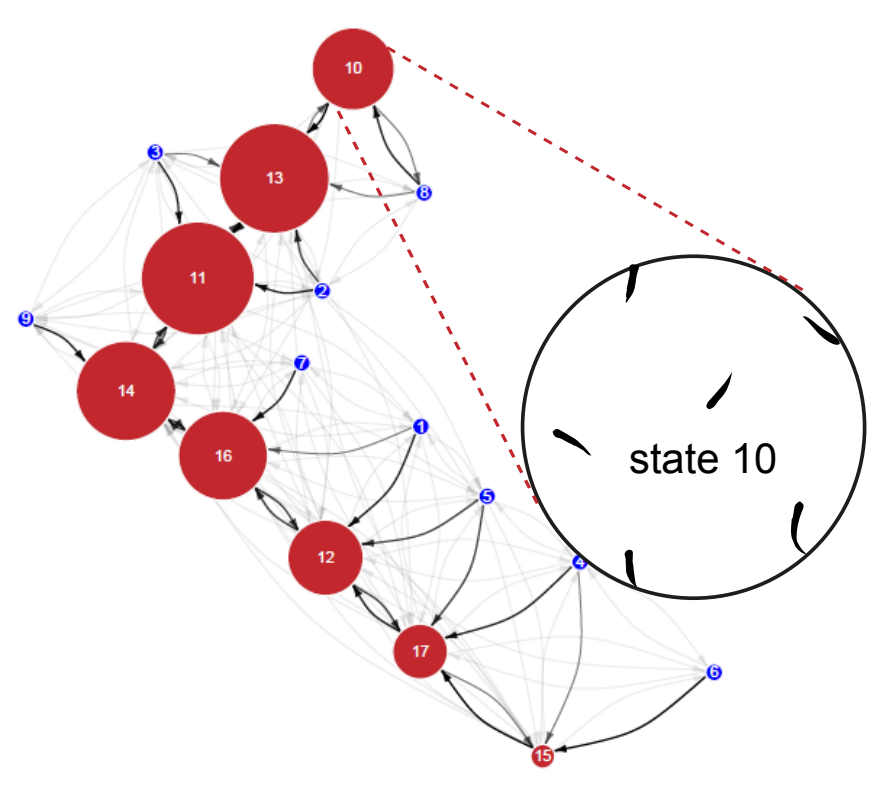

$n=22$ groups

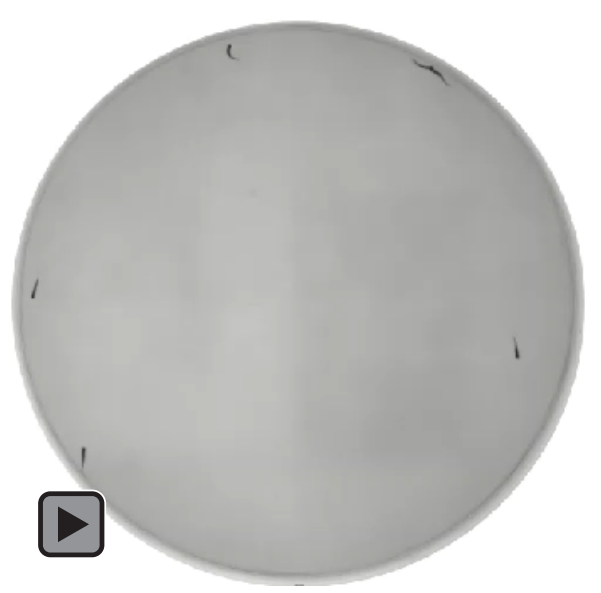

Figure 2. Ethograms of (a) wild-type fish; (b) immp2/-/- mutant fish, showing enhanced cohesion (note that dispersed polarized state 8 and unpolarized state10 are missing); (c) scn 1/ab+/- mutant fish, showing enhanced dispersion. Videos can be accessed by clicking where shown, or via Supplemental Video 1 (wild-type), Supplemental Video 2 (immp2l-/-), and Supplemental Video 3 (scn1lab $+/-)$. Usage ratios are quantified in Supplemental Figure 3 and 4. (wild-type, $\mathrm{n}=33$ groups; immp2l-/-, n=9 groups; scn1/ab+/-, $\mathrm{n}=22$ groups). Color bar indicates transition probability: [0 0.85$]$. 
a bi\&lidivarearint doi: https://doi.org/10.1101/350314; this version posted June 19, 2018. The copyright holder for this preprint (which was not certified by peer review) is the author/funder. All rights reserved. No reuse allowed without permission.
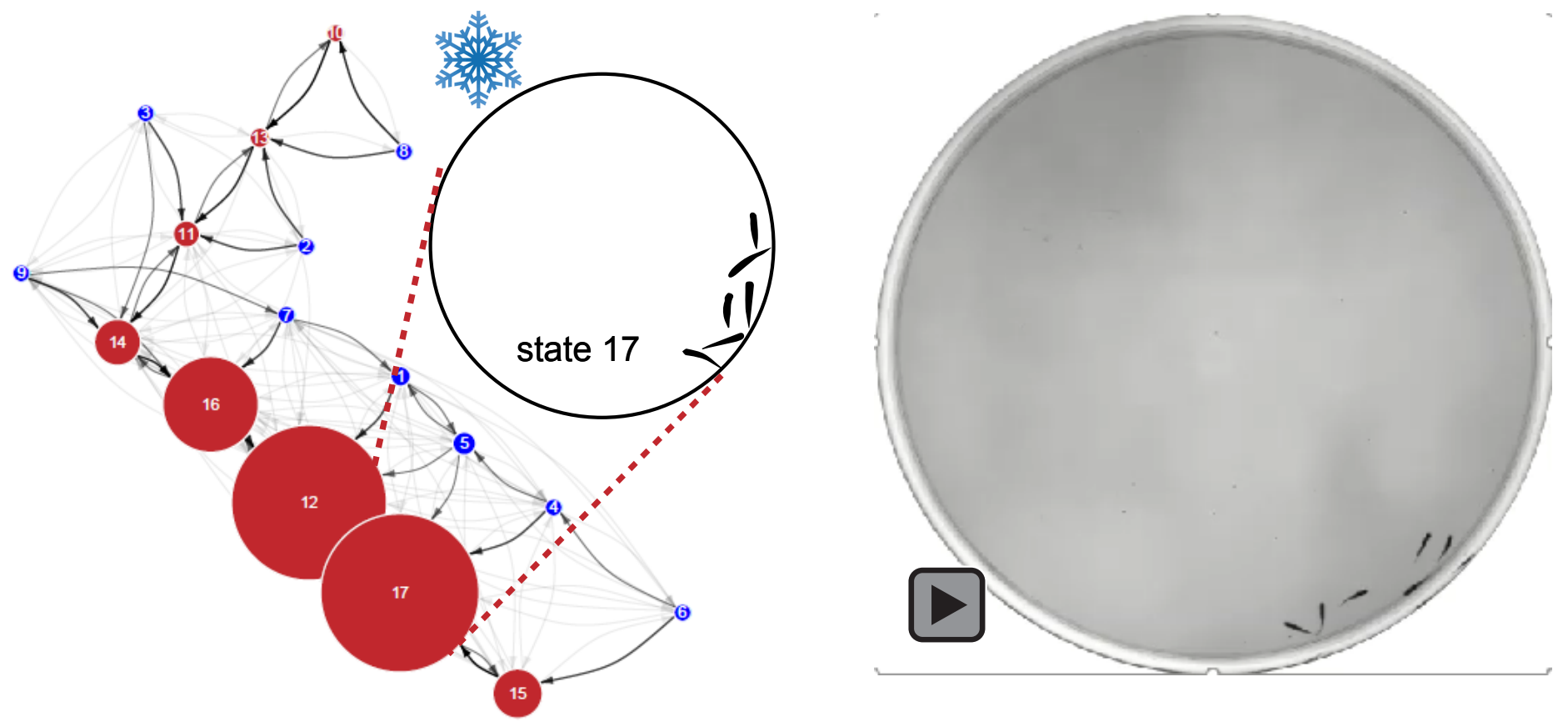

$$
\mathrm{n}=8 \text { groups }
$$

b

wild-type

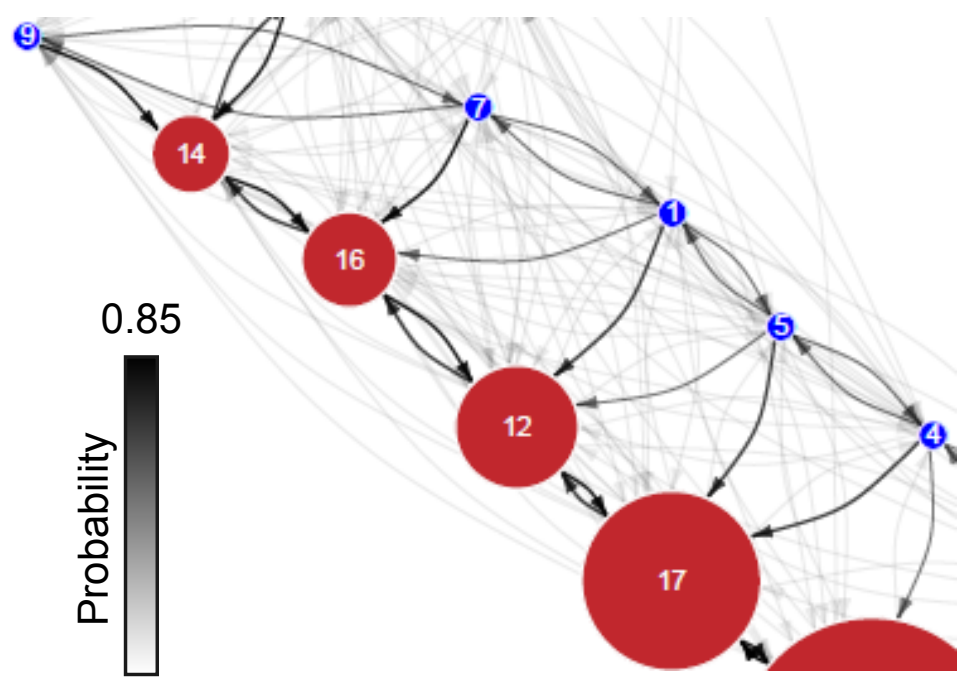

$\mathrm{n}=33$ groups adra1aa-/-

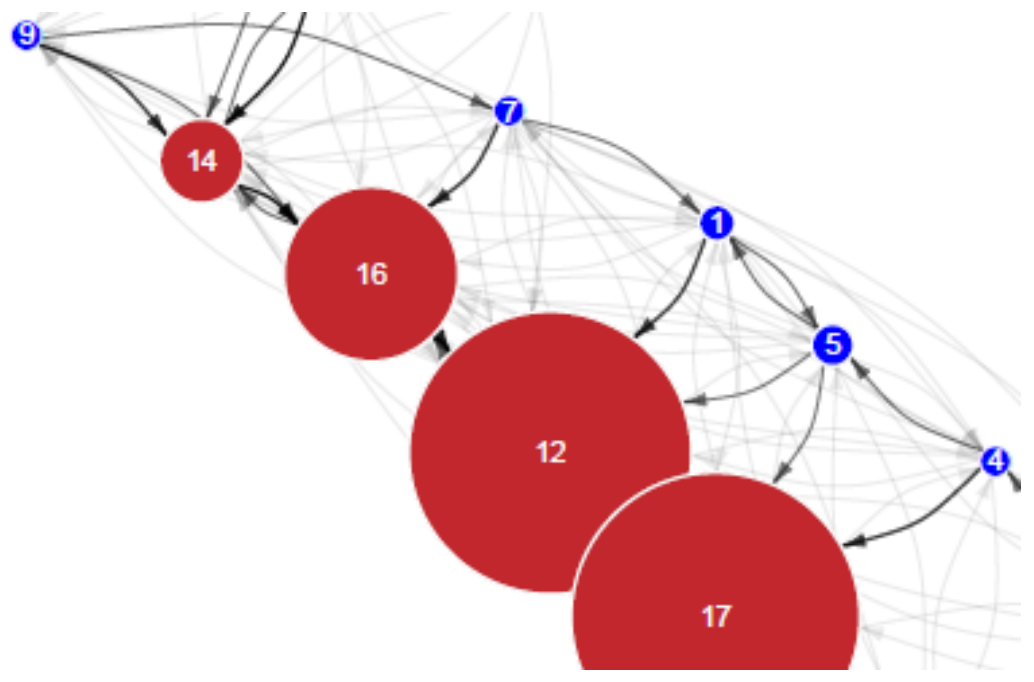

$n=8$ groups

Figure 3. (a) Ethogram of adra1aa-/- mutant fish ( $n=8$ groups) showing reduced transitions. Video (Supplemental Video 4) is clickable, and shows bouts of "freezing". (b) blow-ups of the lower part of the ethograms, comparing wild-type to adra1aa-/- fish, to illustrate the relative paucity of transitions between states of adra1aa-/- fish. Color bar indicates transition probability: [0 0.85]. 
ethogram of Figure 2b $(\mathrm{n}=9$ groups, $\mathrm{p}<0.001$; Mann-Whitney U-test; Supplemental Figure 6a). This is the only mutation to completely abolish individual states (states 8 and 10 are missing). In the contrast with scn1lab+/-the two missing states are the most dispersed polarized and unpolarized states._The fish can move dynamically between the limited states, and rarely polarize (usage ratios shown in Supplemental Figure 3). Cross validation of this robust phenotype was performed by multi-class SVM (Prediction Accuracy (PA) =0.95; Leave-OneOut; Supplemental Figure 5). These fish feed well and spawn (Figure 4), and in the home tank movements are indistinguishable from normal.

Enhanced dispersion Fish mutant in the Nav1.1 sodium channel, scn1lab+/-, remain dispersed throughout the chamber, rarely aggregating, as shown in Figure 2c and its attached video (Supplemental Video 3). This is represented by the shift in predominant states of the ethogram to states to the upper right compared to wildtype ( $\mathrm{n}=22$ groups; $\mathrm{p}<0.001$; Mann-Whitney U-test; Supplemental Figure 6b). In fact, the states lost in immp2l-/- (states 8 and 10) are enhanced in scn1lab+/-. Cross validation of this robust phenotype was performed by multi-class SVM (PA=0.88; Leave-One-Out; Supplemental Figure 5). The group centroid speed, pairwise distance, and polarization have little correlation with each other (Supplemental Figure 7). The fish feed, spawn and grow normally.

Enhanced freezing Fish mutant in the adrenergic receptor, adra1aa-/-, have many more periods of inactivity than do wild-type, especially towards the end of the half hour test. The state usage of the selected top two states for each comparison is not very distinct from wild type ( $\mathrm{n}=8$ groups; Tight shoaling: $\mathrm{p}=0.59$; Medium schooling: $\mathrm{p}=0.86$ and Dispersion: $\mathrm{p}=0.07$; Supplemental Figure 6a-b) as shown in Figure 3a. Compared to wild-type, adra1aa-/- mutant fish tend to have significantly more "freeze" motifs in the last third of the recording time $(\mathrm{p}<0.001)$, as shown in Supplemental Figure 6c. . This difference in dynamics is shown by the relative paucity of arrows in the ethogram blow up of Figure 3b and the attached video (Supplemental video 4). Cross validation of this robust phenotype was performed by multi-class SVM (PA=0.92; Leave-One-Out; Supplemental Figure 5). The fish feed and spawn normally, and their behavior in the home tank is indistinguishable from wild-type.

\section{Effects on other behaviors}

We were curious as to whether the mutations that perturb collective activity also markedly affect motor control or vision. In terms of motor function, we measured speed, the frequency of acceleration, and turns of individual fish in mating 
177 behavior. The three mutations with collective behavioral phenotypes noted above 178 can reach the same level or even higher in all three motion measurements 179 compared to wild type fish. We explored mating, another social behavior, a dance 180 with strong and close proximate interactions, on the assumption that subtle visual or 181 motor defects would perturb the effectiveness of this rapid and highly stereotyped 182 interaction (Nasiadka \& Clark, 2012; Spence, Gerlach, Lawrence, \& Smith, 2008). It 183 also has a quantitative output, i.e., number of fertilized eggs. The spawning 184 consequences of the mutations were modest, immp2l-/- and adra1aa-/- slightly 185 increasing and scn1lab+/- slightly reducing spawning (Figure 4). Hence the genes 186 that dictate collective behavior have only modest effects on the individual 187 behaviors or mating capabilities that we measured. a

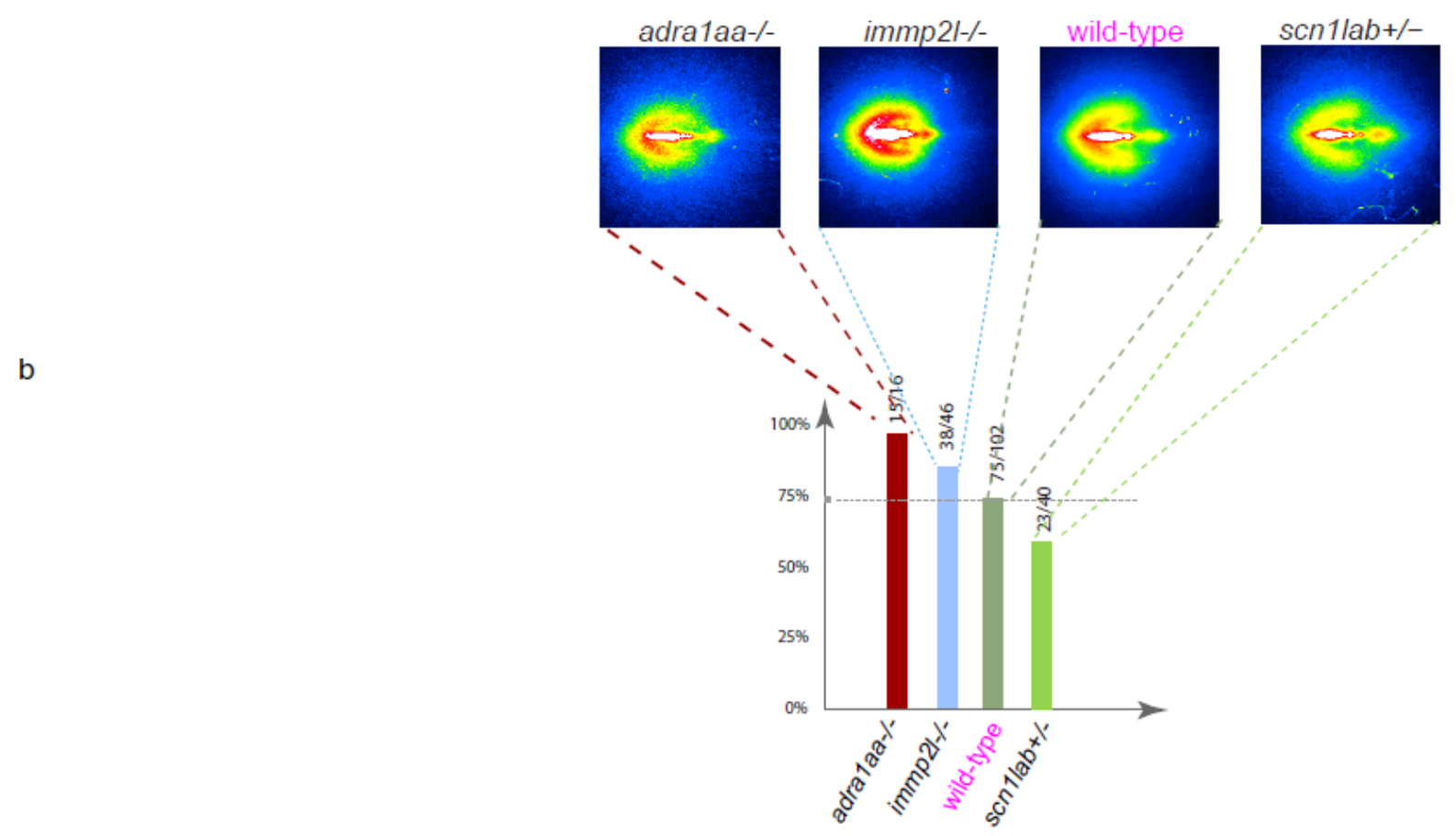

Figure 4. Courtship and spawning is relatively preserved in mutant fish. (a) Positional maps of chasing behavior, with female fish centered facing right and male fish chasing position frequency plotted as a heat map, shows chasing behavior is relatively retained in the mutations of interest. (b) Success of courtship behaviors from mutants and wild-type ranked by the ratio of pairs yielding fertilized embryos, showing modest effects on spawning of the mutations of interest.

\section{Discussion}

Darwin (Darwin, 1871) and Tinbergen (Tinbergen, 1952) speculated as to why 
some species live in groups and others as solitary animals. Presumably, the benefits of living and moving in a collective provide some advantages (Couzin, 2009; Sumpter, 2010). It is believed that fish school and birds flock in part to help avoid or confuse predators, to improve foraging, and to reduce energy expenditure, especially during long haul migration. But such activities come at a cost to individuals, for example, the need to share food and the exposure of those positioned on the edge of the school to attack (Krause, 1994). Hence, how group activity has arisen though evolution, where selection acts on the genome of the individual, has been a source of intense debate.

Here we show that collective behavior is stereotypical and can be described as the consequence of time spent in, and transitions between, 17 different states, each lasting around. By clustering cost curve (Supplemental Figure 8b), we found these were the fewest number of states needed to define the full repertoire of collective actions for wildtype and mutant fish.

We found that single gene changes can modify collective activity in interpretable manner, using the same states. In other words, mutations did not add states. Mutation in the mitochondrial membrane peptidase 2-like gene immp2l increases the tendency to aggregate in tight cohesion, and caused the loss of two highly dispersed states. Mutation in the sodium channel Nav1.1 gene scn1lab appears to prevent any group interaction, abolishing cohesion and polarization. Mutation in the adrenergic receptor gene adra1aa reduces the transitions between states, and causes the animals often to freeze. Freezing and tight cohesion both are seen as responses to threat and conspecific injury (Chicoli \& Paley, 2016; Faustino, Tacao-Monteiro, \& Oliveira, 2017; Rennekamp et al., 2016).

It is noticeable that these effects correspond to some of the forces (repulsion, attraction, and spontaneity) applied to individual particles in mathematical models to generate coordinated movements of groups (Katz et al., 2011; Krause et al., 1999; Li \& Xiao, 2010; Sumpter, 2010). (We have not seen, so far, mutations with selective effects on polarization, a fourth such force). In these models, individual fish need only to manifest these forces between immediate neighbors for the larger scale behavior to emerge as a consequence.

One might argue that widespread changes in neural function could affect a range of behaviors. Although we cannot rule out subtle changes in these parameters, these mutations did not dramatically affect vision or motor function. Of course, 
other sensory cues might be changed, such as pheromones, so critical to mating (Spence et al., 2008; Yabuki et al., 2016) and to aggregation and dispersion of $C$. elegans (Greene, Dobosiewicz, Butcher, McGrath, \& Bargmann, 2016). However, mating remains intact, in terms both of the ornate dance and number of fertilized eggs produced, and we noted no deficiencies in feeding. We also recognize that different environments will elicit distinct behaviors, as will other variables, such as numbers of fish participating (Tunstrom et al., 2013; Yates et al., 2009) and familiarity of the fish with the test chamber. In our studies, the assay was done on fish which had never experienced the test chamber before.

To our knowledge these are the first genes shown to regulate group collective for other attributes. A mutation in the Inner Mitochondrial Membrane Peptidase 2-Like Gene (immp2l) reduces fertility (Lu et al., 2008). One where there is some similarity is haploinsufficiency of the voltage-gated sodium channel, Nav1.1 (Han et al., 2012), which causes hyperactivity and loss of preference for another mouse over empty chambers and curiosity about a novel mouse.

We selected these genes for mutation because they have been reported, with differing degrees of certainty, to have genetic association with autism and schizophrenia, disorders marked by impairments in social interaction. Rare genetic mutations of the IMMP2L gene have been identified in Tourette syndrome and autism spectrum disorders (ASD), and this gene is in the 7q31 translocation region of a Tourette syndrome cohort (Bartnik et al., 2012; Petek et al., 2001). Haploinsufficiency of Nav1.1 in human causes Dravet syndrome, with autism proceeded in infancy by seizures. The gene for the adrenergic receptor ADRA1A is in a large genomic region associated with autism, but no behavioral role has previously been speculated.

Of course, whether these mutations are good models for behavioral deficits in humans remains to be seen. Heterozygous mutation of Nav1.1 in humans, fish, and mice all reduce social interactions. But with the exception of Nav1.1, we have evaluated homozygous effects of mutations, while in humans the mutations are studied in patients with heterozygous deficiencies. To the extent there is duplication of function that accompanies the duplication of many genes in teleost compared to humans or mice (Meyer \& Schartl, 1999; Sztal, McKaige, Williams, Ruparelia, \& Bryson-Richardson, 2018) certain effects may be compensated. In fact, adra1a is split into adra1aa and adra1ab, knockout of each which causes enhanced freezing, suggesting that there may be duplication of function and 
compensation. This may also explain why certain candidate autism genes with striking behavioral disturbances when knocked out in mice, such as dlg4a and shank3b (Feyder et al., 2010; Peca et al., 2011), have less striking phenotypes in the fish. Additionally, our assays observe only a small biopsy of potential collective activities under specific controlled circumstances. Nonetheless, zebrafish are amenable to chemical screens for restoration of mutant to normal phenotype (Peterson et al., 2004),

It seems remarkable that some of the genes we discovered here to regulate collective behavior of fish also have been proposed to affect social behavior of humans. This suggests that, perhaps, the role of genes for social behavior, writ large, is conserved through evolution, of course playing out distinctively in different species. The relative convenience of the fish for therapeutic compound screening (Peterson et al., 2004), may be used to advantage in phenotypic screens for reparative agents for disorders such as autism and schizophrenia.

Materials and methods:

\section{Animals}

Zebrafish (Danio rerio; AB strain) were housed with mixed gender in 3L tanks on a recirculating Aquatic Habitats facility (Custom design, Pentair, USA). The fish were kept at a density of 12 fish per 3L tank. Facility water temperature was kept at $28 \pm 0.5^{\circ} \mathrm{C}$, and water sourced from deionized water conditioned with sodium bicarbonate (Catalog \#SC12-Pentair, USA) and Instant Ocean sea salt (Catalog \#IS160-Pentair, USA) to a $\mathrm{pH}$ of $7.2 \pm 0.5$ and conductivity of $420 \pm 50 \mu \mathrm{S}$. Fish were maintained on a 14-hour light/10-hour dark cycle with light turning on at 07:00 AM. Fish were fed a diet of brine shrimp (Catalog \#BSEPCA-Brine Shrimp Direct, USA) twice daily and supplemented with flake fish food (Tetramin Catalog\# 98525-Pentair, USA) every other day. All animals were maintained and procedures were performed in accordance with the Institutional Animal Care and Use Committees (IACUC) of NIBR.

\section{Genome-wide CRISPR-Cas9 sgRNA design}

Because the current work was carried out in the $\mathrm{AB}$ strain and the public genome assembly (GRCz11) is based on TU strain, we established a new genome assembly for the $\mathrm{AB}$ strain (unpublished, sequences available upon request). For designing CRISPR/Cas9 sgRNAs, we re-trained sgRNA-efficiency models using Random Forest and Naïve Bayes methods based on previously published sgRNAs. 1280 sgRNAs sequences and their editing efficiencies were obtained 
316 from previous study in TU-strain by Moreno-Mateos, M. A. et al., (Moreno-

317 Mateos et al., 2015), and mapped to the AB genomic sequence. About 150

318 features were used to train the two Machine Learning models, Random Forest and 319 Naïve Bayes, in classification mode, including genomic strand of sgRNA, GC\%, 320 identity of $\pm 4 \mathrm{bp}$ of sgRNA targeting sequence, thermodynamics parameter, $\Delta \mathrm{G}$ 321 of sgRNA-genomic DNA heteroduplex for sliding windows of different sizes 322 (Sugimoto, 1995), the free-energy of stem-loops 1, 2, and 3, tetraloop, repeat-anti 323 repeat and linker structures of the full-length sgRNA predicted by UNA-fold 324 (Hiroshi Nishimasu, 2014; Markham \& Zuker, 2008) and etc. A training accuracy 325 of $>0.7$ was achieved by both models, and the efficacies of all sgRNAs in the AB genomic sequence was predicted. Guide RNAs with high predicted efficacy were selected to target the 5' end of the coding exons or Pfam domains of the genes in current study (for the full gene list, citations for disease indications, please see Supplemental. Table 1 and for the guide RNA sequences used for CRISPR-cas9, please see Supplemental. Table 2).

\section{Micro-injections, CRISPR/Cas9 mutant founder identification}

sgRNAs were synthesized using $\mathrm{T} 7$ in vitro transcription using the MEGAshortscript ${ }^{\mathrm{TM}}$ T7 Transcription Kit (ThermoFisher, AM1354). Cas9/sgRNAs were co-injected into 1-cell stage fertilized zebrafish embryos. Conditions were optimized to maximize the CRISPR efficiency in our settings. High indel rates (>90\%) were usually observed in fully developed embryos injected with $500 \mathrm{ng} / \mu \mathrm{L}$ Cas9 protein (PNABio, CP01) and $125 \mathrm{ng} / \mu \mathrm{L}$ sgRNA purified with MEGAclearTM-96 Transcription Clean-Up Kit (ThermoFisher, AM1909).

Indel rate in the injected zebrafish were measured using NGS at 2 days post fertilization (dpf). PCR were performed on the genomic DNA extracted from zebrafish larvae using the HotSHOT method(Meeker, Hutchinson, Ho, \& Trede, 2007), and NGS libraries for PCR products were generated using Nextera DNA Library Preparation Kit (Illumina, FC-121-1031). Subsequently, Nextera libraries were sequenced on Illumina MiSeq, and > avg. 2000 reads for each amplicon were obtained. Sequencing reads were mapped to the reference sequence using BLAT (Kent, 2002), and indels were extracted from the .psl file using a bioinformatic pipeline developed in-house (available upon request). The gene editing efficiency in the injected embryos were calculated as the maximum indel rate within the \pm 30 bp regions of PAM sites.

We usually sequence five larvae per CRISPR injected clutch to confirm the gene 
editing efficiency. The rest fish of the confirmed clutch were raised to adulthood and crossed with wild-type $\mathrm{AB}$ fish, and founder fish carrying the desired frameshift mutation were screened from the F1 generation. F1 heterozygotes were inter-crossed, and homozygotes, if viable, were identified and inter-crossed again to obtain sufficient gene knockout fish for behavioral assays. Each CRISPR line was at least an F2 stable line before being run in any assay. All founders and homozygote identification were carried out using fin-clipping PCR, and sequencing the PCR product using NGS as described above. The indel sequence of homozygous mutations and heterozygous mutations that could not be bred to homozygous (scn1lab and slc18a2) are illustrated in Supplementary File 1, and also the predicted protein sequence aligned with that of the wild-type fish.

\section{Experiment setup for behavioral assays}

Attention was paid to ensure fish gender balance and matching of size and age, and to conduct experiments at similar times of days and feeding cycle, and to monitor by video without human presence. All behavioral rooms were fed with water directly from the main fish facility and room temperature and light cycle was consistent with the main facility. The behavioral setups consisted of 20" diameter acrylic circular arena (Custom Design: Acrylic Tank - Clear - 20" OD x 19.25” ID x 8” height - Open Top, Plastic Supply, Inc., USA) filled to a depth of $13 / 4$ " ( 9 L total volume) with system water fed directly from the main housing unit to ensure all water parameters were identical to the housing conditions. The circular arenas were coated on the outside (I00810, Frosted Glass Finish, Krylon, USA) to prevent the fish from being able to see outside of the arenas but to allow IR light transfer. Underneath the tanks were 24 inch adjustable IR panels (with 940 nm IR LEDs made by Shenzhen VICO). Basler Ace 2040-90um Near Infrared (NIR) cameras (Order\#-106541, Graftek Imaging, USA) were mounted 23 inches above the arena to collect a dorsal view of the fish. Infrared long pass filters (Midopt LP780-62, Graftek Imaging, USA) were attached to the lens (Scheider Cinegon 1.9, Graftek Imaging, USA) and were set to an aperture of 6 (Supplemental Figure 9a). All trials were recorded after 10 minutes habituation to allow recovery from any stress due to netting. Briefly, shoaling assays were run with six fish (3 males and 3 females), which were randomly combined from multiple tanks of siblings (with the exception of chd8-/- which had only male homozygous animals so were paired with $3 \mathrm{AB}$ females) (Supplemental Figure 9c). Each trial was a recording of $30 \mathrm{~min}$ at $60 \mathrm{fps}$. Arenas were rinsed clean with system water at the end of the day and put through a cabinet washer (Type: 9LAV65, IWT Tecniplast Inc., Italy) once a week on a hot water only cycle. 
Courtship assays (1 male and 1 female) were all conducted in Aquatic Habitat 2L group mating tanks (Catalog\# Breeder2-Pentair, USA) positioned carefully within arenas used for the above shoaling assays (Supplemental Figure 9b). The main arena was still filled with water to ensure all fish's safety as they infrequently escape the mating tanks, which remain lidless due the need to eliminate optical obstructions. Image size was fitted to exactly cover the two tanks in the overnight assays (Supplemental Figure 9d). Three 30-min videos were recorded for each pair of animals: the first recording was initiated at 16:00 PM while fish were separated by a clear divider; the second recording at 22:00 PM while the lights were off and divider still in place; the third recording was done at 08:00 AM (the following day) with the divider removed. After each recording, spawning records were documented and confirmed only if more than 10 embryos survive till $1 \mathrm{dpf}$ with normal course. All mating tanks used in assays were washed daily in under counter washers (Type: GG05/Model PG 8583; Miele AG, Germany).

\section{Automated data collection}

We used a virtual instrument console designed within LabVIEW (National Instruments, USA) to control all cameras. The acquisition software was designed with four prioritized functions: 1) Saving the recorded video; 2) Logging all relevant metadata accurately and automatically; 3) Enabling real time user visualization, verification and modification; 4) Generating associated files to streamline downstream analysis.

Non-default parameters were set within the NI-MAX (Measurement and Automation Explorer) including dimensions and offsets for centering (1880x1880 pixels (offset 84/84) for Shoaling assays, 1000x1000 pixels (offset 420/600) for Courtship assays) and frame rate (60 fps). One workstation (X2D65UT\#ABA, Z440, Hewlett Packard Inc., USA) was dedicated for simultaneous recordings of two USB3.0 Basler ACE cameras. The resulting videos which are approximately 360 GB each for Shoaling assays, and 108 GB each for Courtship assays are saved on a local 4x2 TB SSD RAID0 (Samsung EVO 850, B\&H Photos Inc., USA). All cameras were named uniquely to allow us to trace back which videos are generated by corresponding setups.

Relevant metadata includes time of trial, user, and fish information (genetic background, age, genetic knockouts, genotype, compound treatment (concentration/duration) and other manipulations), and assay information (duration, fps, number of male and female fish). All software developed in house is available upon request, excluding licenses. 


\section{Data preprocessing}

The processing involved building of fingerprint libraries to distinguish fish accurately even after repeated crossings, using locomotion quantifications such as trajectory, angles, speeds, pairwise distances, and convex hull area. (software based on MATLAB 2013a; MathWorks, U.S.A.) inspired by Perez-Escudero et al. (Perez-Escudero, Vicente-Page, Hinz, Arganda, \& de Polavieja, 2014). In addition, we used supervised behavioral annotation of simple motifs, such as nonsocial turn, dive, rest, and cruise, or social chase, cross, and parallel rotation, with previously trained classifiers as described by Kabra et al (Kabra et al., 2013). These processing procedures are integrated into an overarching program that manages data dependencies to automatically start the next stages when ready without needing additional user inputs (scripts available upon request).

\section{Unsupervised collective behavior state hierarchical structure clustering}

For this analysis we used 5 frame epochs. Polarization was determined by the swimming direction angle. For the $i^{\text {th }}$ fish in epoch $n$, this was represented by the tangent angle of the $i^{\text {th }}$ fish's trajectory from the $1^{\text {st }}$ frame of the $n^{\text {th }}$ epoch to the $1^{\text {st }}$ frame of the $n+1^{\text {th }}$ epoch in the Cartesian coordinates where the trajectory was represented. If the standard deviation of the angles of the six fish in an epoch was lower than the threshold 0.6 radians and the minimum swimming speed of the 6 fish in the epoch faster than 1 inch per second, the epoch was classified as swimming”.

The pairwise distances among the six fish's centroids was calculated from the $1^{\text {st }}$ frame to represent the pairwise distances in each epoch. The pairwise distances within each epoch was ranked in a descend order. A principle component analysis (PCA) dimension reduction was applied to the ranked pairwise distance matrices (with a dimension of $C_{6}^{2}$ by number of epochs) in polarized swimming and unpolarized swimming epochs, respectively. The top 2 components that capture more than 95\% of variance were kept and fuzzy $C$-means clustering was implemented on the top 2 PCA components with $k=9$ states, for polarized swimming and $k=8$ for unpolarized swimming components, respectively. The flow chart of the unsupervised learning approach we performed is shown in Supplemental Figure 9a. A majority filter is applied to the clustered labels to eliminate cases that only a single epoch has a different label from previous epoch labels and post epoch labels, since there is less interest in super short states with 
476 duration less than $1 / 6$ seconds. Change points identified state block duration 477 distribution is shown in Supplemental Figure 9. The mean of block duration is $478 \quad 0.78$ seconds with a standard deviation of 0.96 seconds.

479

480

A t-distributed stochastic neighbor embedding (t-SNE) (Laurens van der Maaten, 2008) plot was used to visualize the high dimensional data in a two dimensional space for the wild-type fish collective behavior (Supplemental Figure 10a-b). The collective behavior pattern is shown by clickable videos for the selected states.

The $k$ values were determined by optimizing the clustering cost (D T Pham, 2005) (Supplemental Figure 9b). Moreover, to increase the resolution of the state quantification, the state number was pushed to $k=50$ for both polarized and unpolarized swimming. The overall results remain consistent.

Self-Organizing map (SOM) clustering and k means were applied as orthogonal approaches to fuzzy $C$-means. The performance of SOM and $\mathrm{k}$ means is equivalent to fuzzy $C$-means (data not shown). All the quantifications are implemented in Matlab R2014a (Mathworks. Inc, U.S.A.). Figure 1 to 3 and supplementary figure $1,2,3,5$, and 10 were plotted in $R$ 3.4.1, the rest figures were plotted in Matlab. Silhouette analysis was performed for both polarized and un-polarized swimming to ensure no significant over classification (data not shown). The Matlab and $R$ codes to replot all the figures in this manuscript are available upon request.

\section{Ethogram visualizations}

Ethograms are used to represent the different states that occur during collective behaviors. The ethogram indicates the frequency and transition probability with which one state is followed by another state (Anderson \& Perona, 2014; Dankert et al., 2009). To represent the relationship among behavioral states and probability distribution of states using the ethogram, two type of analysis were performed. First, we computed the one-step transition probability which is the conditional distribution of the current state given by the previous state (Bishop, 2006). Second, we computed the probability distribution of states which is the frequency of the occurrence. In practice, The ethogram is generated by igraph $R$ package (Csardi, 2006). In the ethogram, the edge and vertex are corresponding to the onestep transition probability and probability distribution of states, respectively. The thicker and darker the edge is, the higher transition probability is; and the bigger the vertex is, the higher the state appearance probability is. All attributes (e.g., vertex color, vertex size, arrow size, edge size, and edge curvature, provided in 
the scripts) were adjusted by parameters of the package to generate current figures.

\section{Kullback-Leibler divergence}

Kullback-Leibler divergence (relative entropy) (Kullback \& Leibler, 1951) is a mathematical method to measure the differences of two probability distributions. The difference $D$ is a function of the two discrete probability distributions $P$ and $Q$ as shown in the following equation (1):

$$
D(P \| Q)=\sum_{i} P(i) \log \frac{P(i)}{Q(i)}
$$

Kullback-Leibler divergence was applied to the polarized and unpolarized state usages, respectively. The differences between each mutation and wild type were ranked in descending order (Supplementary File 2) and the top ranked mutations were selected.

\section{Supervised behavioral phenotype category classification}

\section{Feature extraction}

State usage, transition matrix and swimming speed in each trial are employed as features (also called measurements or attributes), including the averaged aggregation (unpolarized) state usages, dispersion (unpolarized) state usages, schooling (polarized) state usages, overall state usages, and transition matrix. Speed features include mean speed of all the 6 fish each trial in the last 10 minutes of the recording, number of stop epochs from all 6 fish in each trial. PCA then is implemented to reduce the dimensionality of the feature space. Top 12 PCs are kept capturing above 95\% of the variance. In addition, an alternative feature pool is tested, including only swimming speed distribution in each unpolarized state from all the trials. PCA dimensional reduction is followed and 3 top PCs are selected to capture $95 \%$ variance of the data.

\section{Support vector machine}

The classifier we implement is the support vector machine (SVM) (Vapnick, 1995), which is widely used for pattern classification (Ma, Randolph, \& Drish, 2001). For the classical binary formulation of SVM classification, the output of an SVM classifier yields:

$$
\begin{aligned}
& y=\operatorname{sign}(f(x)), \\
& f(x)=\sum_{i=1}^{N_{S V}} \alpha_{i} y_{i} k\left(x, x_{i}\right)+b,
\end{aligned}
$$

where $\alpha_{i}$ are the Lagrange multipliers from solving the quadratic optimization 
problem, $y_{i}$ are the class targets, $x_{i}$ are the input data points, $k\left(x, x_{i}\right)$ is a kernel function (Chang, 2011), $b$ is the bias, and $N_{S V}$ is the number of support vectors. Linear kernel is used here since it is more capable of avoiding possible overfitting in the classification than other kernels (Keerthi \& Lin, 2003).

To extend a binary classification to a multi-class classification problem such as the problem at hand, several binary SVM classifiers need to be utilized. One-againstone classification method trains $k(k-1) / 2$ binary classifiers (where $k$ is the number of classes) to solve $k$ class classification problem. During prediction the class with the most votes becomes the winner. One-against-one outperforms other multi-class SVM classification methods (Hsu \& Lin, 2002) and is the default choice in the libSVM package (Chang, 2011) that has been utilized in this study.

\section{Leave-one-out}

The Leave-One-Out (LOO) (Efron, 1993) validation method is used to evaluate the performance of the classifiers described in this study. Random one trial from each category is taken as the testing data and the remaining as the training data to train SVM multi-class classifiers. This procedure was applied for 100 times (most trial numbers are less than 10) by randomly choosing training and testing trials. All the test results are then combined into a cumulative confusion matrix.

\section{Acknowledgements}

We thank Ricardo Dolmetsch, Rainer Friedrich, Joseph Loureiro, Mark Borowsky, Brant Peterson, Gerald Sun, Lingling Shen, Ajeet Singh, Vibhas Aravamuthan, Stephen Litster, Guangliang Wang, and Jian Fang for discussion. We also want to thank Gerlinde Wussler, Kara Moloney, Meghan Aguirre, Franki Vetrano-Olsen, KarenJ Lee, Haley Clark, Johnathan Tobin, and Joseph Beaton for their support in the fish facility, also Stacey Gearin, Stephanie Wiessner, and Jessica Garver for CRISPR injection work, Caroline Fawcett for fish database management; thank Michael Steeves and Michael Derby for their help in computation resource; thank Michael Paolucci and Aaron Bickel for their help in hardware customizing; thank Andrea Schwarz and Lauren Goldfinger for project coordination. X.X. is supported by NIBR postdoctoral scholar program. Funding is provided by the Novartis Institutes for Biomedical Research (NIBR).

\section{Competing interests}


MCF: Consultant to NIBR; Boards of Directors of Semma Therapeutics and Beam Therapeutics; SAB of Tenaya Therapeutics.

\section{Reference}

Anderson, D. J., \& Perona, P. (2014). Toward a science of computational ethology. Neuron, 84(1), 18-31. doi:10.1016/j.neuron.2014.09.005

Baier, H. (2000). Zebrafish on the move: towards a behavior-genetic analysis of vertebrate vision. Curr Opin Neurobiol, 10(4), 451-455.

Bartnik, M., Szczepanik, E., Derwinska, K., Wisniowiecka-Kowalnik, B., Gambin, T., Sykulski, M., . . . Stankiewicz, P. (2012). Application of array comparative genomic hybridization in 102 patients with epilepsy and additional neurodevelopmental disorders. Am J Med Genet B Neuropsychiatr Genet, 159b(7), 760-771. doi:10.1002/ajmg.b.32081

Bishop, Christopher (2006). Pattern Recognition and Machine Learning: Springer.

Brockerhoff, S. E., Hurley, J. B., Janssen-Bienhold, U., Neuhauss, S. C., Driever, W., \& Dowling, J. E. (1995). A behavioral screen for isolating zebrafish mutants with visual system defects. Proc Natl Acad Sci U S A, 92(23), 10545-10549.

Chang, Chih-Chung, Lin, Chih-Jen. (2011). LIBSVM: A library for support vector machines. ACM Trans. Intell. Syst. Technol, 2(3), 1-27.

Chicoli, Amanda, \& Paley, Derek A. (2016). Probabilistic information transmission in a network of coupled oscillators reveals speed-accuracy trade-off in responding to threats. Chaos, 26(11), 116311. doi:10.1063/1.4966682

Chuang, K. S., Tzeng, H. L., Chen, S., Wu, J., \& Chen, T. J. (2006). Fuzzy c-means clustering with spatial information for image segmentation. Comput Med Imaging Graph, 30(1), 9-15. doi:10.1016/j.compmedimag.2005.10.001

Couzin, I. D. (2009). Collective cognition in animal groups. Trends Cogn Sci, 13(1), 36-43. doi:10.1016/j.tics.2008.10.002

Csardi, Gabor, \& Nepusz, Tamas. (2006). The igraph software package for complex network research. InterJournal, Complex Systems, 1695(5), 1-9.

D T Pham, S S Dimov, C D Nguyen. (2005). Selection of K in K-means clustering Journal of Mechanical Engineering Science, 219(1), 103-119.

Dankert, H., Wang, L., Hoopfer, E. D., Anderson, D. J., \& Perona, P. (2009). Automated monitoring and analysis of social behavior in Drosophila. Nat Methods, 6(4), 297-303. doi:10.1038/nmeth.1310

Darwin, Charles. (1871). The Descent of Man, and Selection in Relation to Sex United Kingdom: John Murray.

Efron, B., Tibshirani, R.J. . (1993). An introduction to the bootstrap: Chapman\&Hall.

Faustino, A. I., Tacao-Monteiro, A., \& Oliveira, R. F. (2017). Mechanisms of social buffering of fear in zebrafish. Sci Rep, 7, 44329. doi:10.1038/srep44329

Feng, Y., Dong, F., Xia, X., Hu, C. H., Fan, Q., Hu, Y., . . Mutic, S. (2017). An adaptive Fuzzy C-means method utilizing neighboring information for breast tumor segmentation in ultrasound images. Med Phys, 44(7), 3752-3760. doi:10.1002/mp.12350

Feyder, M., Karlsson, R. M., Mathur, P., Lyman, M., Bock, R., Momenan, R., ... Holmes, A. (2010). Association of mouse DIg4 (PSD-95) gene deletion and human DLG4 gene variation with phenotypes relevant to autism spectrum disorders and Williams' syndrome. Am J Psychiatry, 167(12), 1508-1517. doi:10.1176/appi.ajp.2010.10040484

Friedrich, R. W., Jacobson, G. A., \& Zhu, P. (2010). Circuit neuroscience in zebrafish. Curr Biol, 20(8), R371-381. doi:10.1016/j.cub.2010.02.039 
Granato, M., van Eeden, F. J., Schach, U., Trowe, T., Brand, M., Furutani-Seiki, M., . . Nusslein-Volhard, C. (1996). Genes controlling and mediating locomotion behavior of the zebrafish embryo and larva. Development, 123, 399-413.

Greene, J. S., Brown, M., Dobosiewicz, M., Ishida, I. G., Macosko, E. Z., Zhang, X., . . Bargmann, C. I. (2016). Balancing selection shapes density-dependent foraging behaviour. Nature, 539(7628), 254-258. doi:10.1038/nature19848

Greene, J. S., Dobosiewicz, M., Butcher, R. A., McGrath, P. T., \& Bargmann, C. I. (2016). Regulatory changes in two chemoreceptor genes contribute to a Caenorhabditis elegans QTL for foraging behavior. Elife, 5. doi:10.7554/eLife.21454

Greenwood, A. K., Wark, A. R., Yoshida, K., \& Peichel, C. L. (2013). Genetic and neural modularity underlie the evolution of schooling behavior in threespine sticklebacks. Curr Biol, 23(19), 18841888. doi:10.1016/j.cub.2013.07.058

Han, S., Tai, C., Westenbroek, R. E., Yu, F. H., Cheah, C. S., Potter, G. B., . . Catterall, W. A. (2012). Autistic-like behaviour in Scn1a+/- mice and rescue by enhanced GABA-mediated neurotransmission. Nature, 489(7416), 385-390. doi:10.1038/nature11356

Han, S., Taralova, E., Dupre, C., \& Yuste, R. (2018). Comprehensive machine learning analysis of Hydra behavior reveals a stable basal behavioral repertoire. Elife, 7. doi:10.7554/eLife.32605

Hiroshi Nishimasu, F. Ann Ran, Patrick D. Hsu, Silvana Konermann, Soraya Shehata, Naoshi Dohmae, Ryuichiro Ishitani, Feng Zhang, and Osamu Nureki. (2014). Crystal Structure of Cas9 in Complex with Guide RNA and Target DNA. Cell, 156(5), 935-949.

Hong, W., Kennedy, A., Burgos-Artizzu, X. P., Zelikowsky, M., Navonne, S. G., Perona, P., \& Anderson, D. J. (2015). Automated measurement of mouse social behaviors using depth sensing, video tracking, and machine learning. Proc Natl Acad Sci U S A, 112(38), E5351-5360. doi:10.1073/pnas.1515982112

Hsu, C. W., \& Lin, C. J. (2002). A comparison of methods for multiclass support vector machines. IEEE Trans Neural Netw, 13(2), 415-425. doi:10.1109/72.991427

Ioannou, C. C., Guttal, V., \& Couzin, I. D. (2012). Predatory fish select for coordinated collective motion in virtual prey. Science, 337(6099), 1212-1215. doi:10.1126/science.1218919

Ioannou, C. C., Morrell, L. J., Ruxton, G. D., \& Krause, J. (2009). The effect of prey density on predators: conspicuousness and attack success are sensitive to spatial scale. Am Nat, 173(4), 499-506. doi:10.1086/597219

Jolles, Jolle W., Boogert, Neeltje J., Sridhar, Vivek H., Couzin, lain D., \& Manica, Andrea. (2017). Consistent Individual Differences Drive Collective Behavior and Group Functioning of Schooling Fish. Current Biology, 27(18), 2862-2868.e2867. doi:10.1016/j.cub.2017.08.004

Kabra, M., Robie, A. A., Rivera-Alba, M., Branson, S., \& Branson, K. (2013). JAABA: interactive machine learning for automatic annotation of animal behavior. Nat Methods, 10(1), 64-67. doi:10.1038/nmeth.2281

Katz, Y., Tunstrom, K., loannou, C. C., Huepe, C., \& Couzin, I. D. (2011). Inferring the structure and dynamics of interactions in schooling fish. Proc Natl Acad Sci U S A, 108(46), 18720-18725. doi:10.1073/pnas.1107583108

Keerthi, S. S., \& Lin, C. J. (2003). Asymptotic behaviors of support vector machines with Gaussian kernel. Neural Comput, 15(7), 1667-1689. doi:10.1162/089976603321891855

Kent, W. J. (2002). BLAT--the BLAST-like alignment tool. Genome Res, 12(4), 656-664. doi:10.1101/gr.229202

Krause, J. (1994). Differential fitness returns in relation to spatial position in groups. Biol Rev Camb Philos Soc, 69(2), 187-206.

Krause, J., Hartmann, N., \& Pritchard, V. L. (1999). The influence of nutritional state on shoal choice in zebrafish, Danio rerio. Anim Behav, 57(4), 771-775. doi:10.1006/anbe.1998.1010 
Kravitz, E. A., \& Huber, R. (2003). Aggression in invertebrates. Curr Opin Neurobiol, 13(6), 736-743.

Kullback, S., \& Leibler, R. A. (1951). On Information and Sufficiency. The Annals of Mathematical Statistics, 22(1), 79-86.

Laurens van der Maaten, Geoffrey Hinton. (2008). Visualizing High-Dimensional Data Using t-SNE. Journal of Machine Learning Research, 9, 2579-2605.

Li, X., \& Xiao, J. (2010). Swarming in homogeneous environments: a social interaction based framework. J Theor Biol, 264(3), 747-759. doi:10.1016/j.jtbi.2010.02.016

Lu, B., Poirier, C., Gaspar, T., Gratzke, C., Harrison, W., Busija, D., . . Bishop, C. E. (2008). A mutation in the inner mitochondrial membrane peptidase 2-like gene (Immp2I) affects mitochondrial function and impairs fertility in mice. Biol Reprod, 78(4), 601-610. doi:10.1095/biolreprod.107.065987

Lukeman, R., Li, Y. X., \& Edelstein-Keshet, L. (2010). Inferring individual rules from collective behavior. Proc Natl Acad Sci U S A, 107(28), 12576-12580. doi:10.1073/pnas.1001763107

Ma, Changxue, Randolph, M. A., \& Drish, J. (2001). A support vector machines-based rejection technique for speech recognition. Paper presented at the Acoustics, Speech, and Signal Processing, Proceedings. (ICASSP '01). 2001 IEEE International Conference on.

Markham, N. R., \& Zuker, M. (2008). UNAFold: software for nucleic acid folding and hybridization. Methods Mol Biol, 453, 3-31. doi:10.1007/978-1-60327-429-6_1

Meeker, N. D., Hutchinson, S. A., Ho, L., \& Trede, N. S. (2007). Method for isolation of PCR-ready genomic DNA from zebrafish tissues. Biotechniques, 43(5), 610, 612, 614.

Meyer, A., \& Schartl, M. (1999). Gene and genome duplications in vertebrates: the one-to-four (-to-eight in fish) rule and the evolution of novel gene functions. Curr Opin Cell Biol, 11(6), 699-704.

Moreno-Mateos, M. A., Vejnar, C. E., Beaudoin, J. D., Fernandez, J. P., Mis, E. K., Khokha, M. K., \& Giraldez, A. J. (2015). CRISPRscan: designing highly efficient sgRNAs for CRISPR-Cas9 targeting in vivo. Nat Methods, 12(10), 982-988. doi:10.1038/nmeth.3543

Muto, A., Orger, M. B., Wehman, A. M., Smear, M. C., Kay, J. N., Page-McCaw, P. S., . . Baier, H. (2005). Forward genetic analysis of visual behavior in zebrafish. PLoS Genet, 1(5), e66. doi:10.1371/journal.pgen.0010066

Nasiadka, A., \& Clark, M. D. (2012). Zebrafish breeding in the laboratory environment. Ilar j, 53(2), 161168. doi:10.1093/ilar.53.2.161

Peca, J., Feliciano, C., Ting, J. T., Wang, W., Wells, M. F., Venkatraman, T. N., . . Feng, G. (2011). Shank3 mutant mice display autistic-like behaviours and striatal dysfunction. Nature, 472(7344), 437442. doi:10.1038/nature09965

Perez-Escudero, Alfonso, Vicente-Page, Julian, Hinz, Robert C., Arganda, Sara, \& de Polavieja, Gonzalo G. (2014). idTracker: tracking individuals in a group by automatic identification of unmarked animals. Nat Meth, 11(7), 743-748. doi:10.1038/nmeth.2994

http://www.nature.com/nmeth/journal/v11/n7/abs/nmeth.2994.html\#supplementary-information

Petek, E., Windpassinger, C., Vincent, J. B., Cheung, J., Boright, A. P., Scherer, S. W., . . Wagner, K. (2001). Disruption of a novel gene (IMMP2L) by a breakpoint in 7q31 associated with Tourette syndrome. Am J Hum Genet, 68(4), 848-858. doi:10.1086/319523

Peterson, R. T., Shaw, S. Y., Peterson, T. A., Milan, D. J., Zhong, T. P., Schreiber, S. L., . . Fishman, M. C. (2004). Chemical suppression of a genetic mutation in a zebrafish model of aortic coarctation. Nat Biotechnol, 22(5), 595-599. doi:10.1038/nbt963

Portugues, R., \& Engert, F. (2009). The neural basis of visual behaviors in the larval zebrafish. Curr Opin Neurobiol, 19(6), 644-647. doi:10.1016/j.conb.2009.10.007

Rennekamp, Andrew J., Huang, Xi-Ping, Wang, You, Patel, Samir, Lorello, Paul J., Cade, Lindsay, . . . Peterson, Randall T. (2016). Sigma-1 receptor ligands control a switch between passive and active threat responses. Nature chemical biology, 12(7), 552-558. doi:10.1038/nchembio.2089 
Rihel, J., Prober, D. A., Arvanites, A., Lam, K., Zimmerman, S., Jang, S., . . Schier, A. F. (2010). Zebrafish behavioral profiling links drugs to biological targets and rest/wake regulation. Science, 327(5963), 348-351. doi:10.1126/science.1183090

Siew, Xiaosheng Peng ; Chengke Zhou ;Donald M. Hepburn ; Martin D. Judd;W. H. (2013). Application of $\mathrm{K}-\mathrm{Means}$ method to pattern recognition in on-line cable partial discharge monitoring IEEE Transactions on Dielectrics and Electrical Insulation, 20(3), 754 - 761

Spedding, G. (2011). Aerodynamics: The cost of flight in flocks. Nature, 474(7352), 458-459. doi:10.1038/474458a

Spence, R., Gerlach, G., Lawrence, C., \& Smith, C. (2008). The behaviour and ecology of the zebrafish, Danio rerio. Biol Rev Camb Philos Soc, 83(1), 13-34. doi:10.1111/j.1469-185X.2007.00030.x

Sumpter, David J. T. (2010). Collective Animal Behavior: Princeton University Press.

Sztal, T. E., McKaige, E. A., Williams, C., Ruparelia, A. A., \& Bryson-Richardson, R. J. (2018). Genetic compensation triggered by actin mutation prevents the muscle damage caused by loss of actin protein. PLoS Genet, 14(2), e1007212. doi:10.1371/journal.pgen.1007212

Tinbergen, N. (1952). The Study of Instict. Oxford, ISBN-0198577222.

Todd, J. G., Kain, J. S., \& de Bivort, B. L. (2017). Systematic exploration of unsupervised methods for mapping behavior. Phys Biol, 14(1), 015002. doi:10.1088/1478-3975/14/1/015002

Torney, C. J., Berdahl, A., \& Couzin, I. D. (2011). Signalling and the evolution of cooperative foraging in dynamic environments. PLoS Comput Biol, 7(9), e1002194. doi:10.1371/journal.pcbi.1002194

Tunstrom, K., Katz, Y., loannou, C. C., Huepe, C., Lutz, M. J., \& Couzin, I. D. (2013). Collective states, multistability and transitional behavior in schooling fish. PLoS Comput Biol, 9(2), e1002915. doi:10.1371/journal.pcbi.1002915

Vapnick, V. (1995). The Nature of Statistical Learning Theory. New York: Springer-Verlag.

Wiltschko, A. B., Johnson, M. J., lurilli, G., Peterson, R. E., Katon, J. M., Pashkovski, S. L., . . Datta, S. R. (2015). Mapping Sub-Second Structure in Mouse Behavior. Neuron, 88(6), 1121-1135. doi:10.1016/j.neuron.2015.11.031

Yabuki, Y., Koide, T., Miyasaka, N., Wakisaka, N., Masuda, M., Ohkura, M., . . Yoshihara, Y. (2016). Olfactory receptor for prostaglandin F2alpha mediates male fish courtship behavior. Nat Neurosci, 19(7), 897-904. doi:10.1038/nn.4314

Yates, C. A., Erban, R., Escudero, C., Couzin, I. D., Buhl, J., Kevrekidis, I. G., . . Sumpter, D. J. (2009). Inherent noise can facilitate coherence in collective swarm motion. Proc Natl Acad Sci U SA, 106(14), 5464-5469. doi:10.1073/pnas.0811195106

Zhu, P., Narita, Y., Bundschuh, S. T., Fajardo, O., Scharer, Y. P., Chattopadhyaya, B., . . Friedrich, R. W. (2009). Optogenetic Dissection of Neuronal Circuits in Zebrafish using Viral Gene Transfer and the Tet System. Front Neural Circuits, 3, 21. doi:10.3389/neuro.04.021.2009 

a $36 d p f$

b $50 d p f$

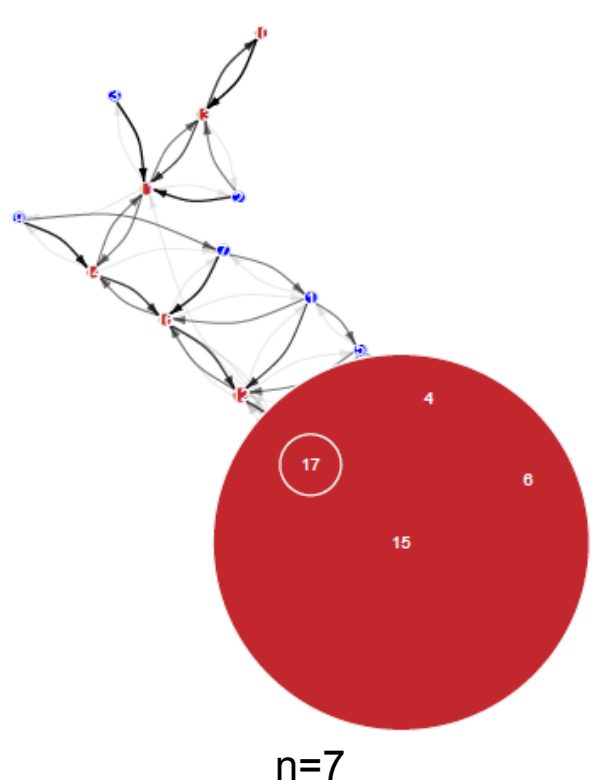

$\mathrm{n}=7$

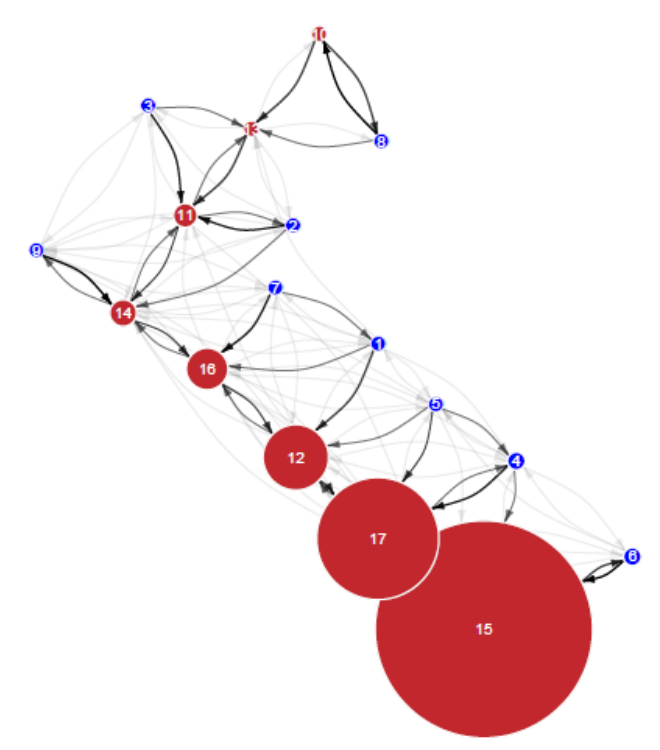

$\mathrm{n}=8$ c $66 d p f$

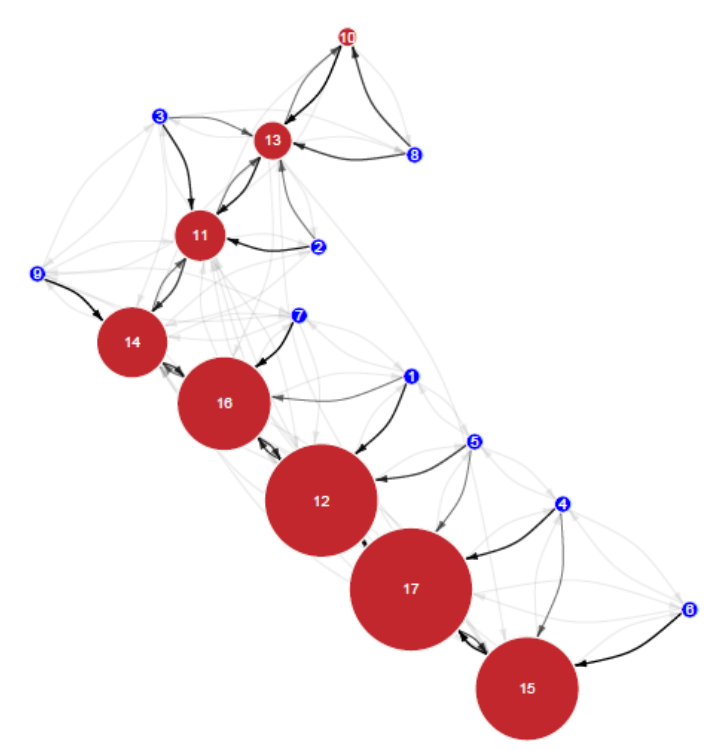

$\mathrm{n}=7$ $d>90 d p f$

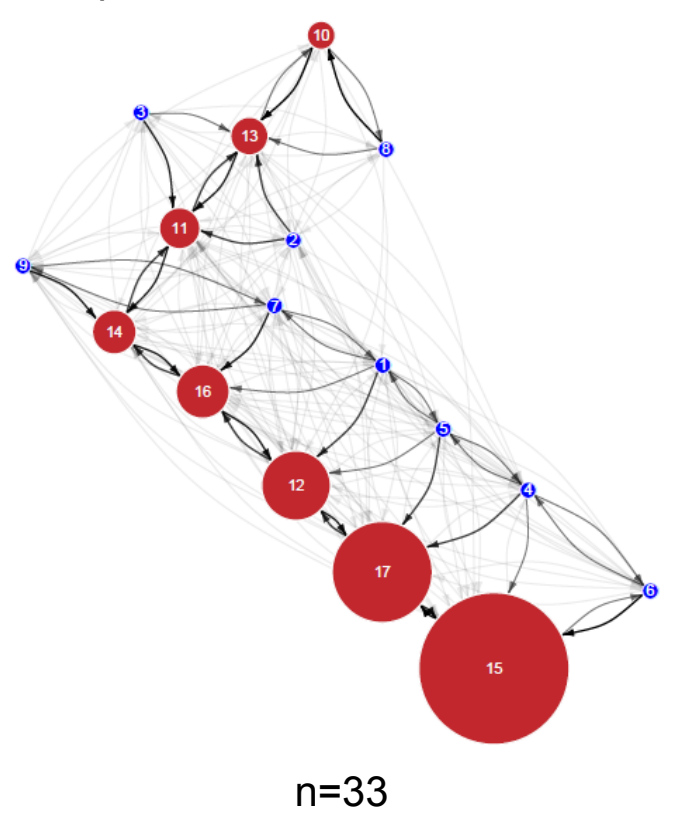

Supplemental Figure 1 Development of collective behavior, showing ethograms of wild-type fish (a) 36 dpf (note that most of the usage concentrates in state 15, the tightest cohesion state, and that the dispersed schooling state 8 is missing; (b) $50 \mathrm{dpf}$ (tight cohesion states 15 and 17 are still the majority of state usage); (c) $66 \mathrm{dpf}$ (the collective behavior state usage is similar to adult fish, although the transitions between states are less dense); (d) adult fish. Collective behavior is not mature until the fish are about 3 months old. 


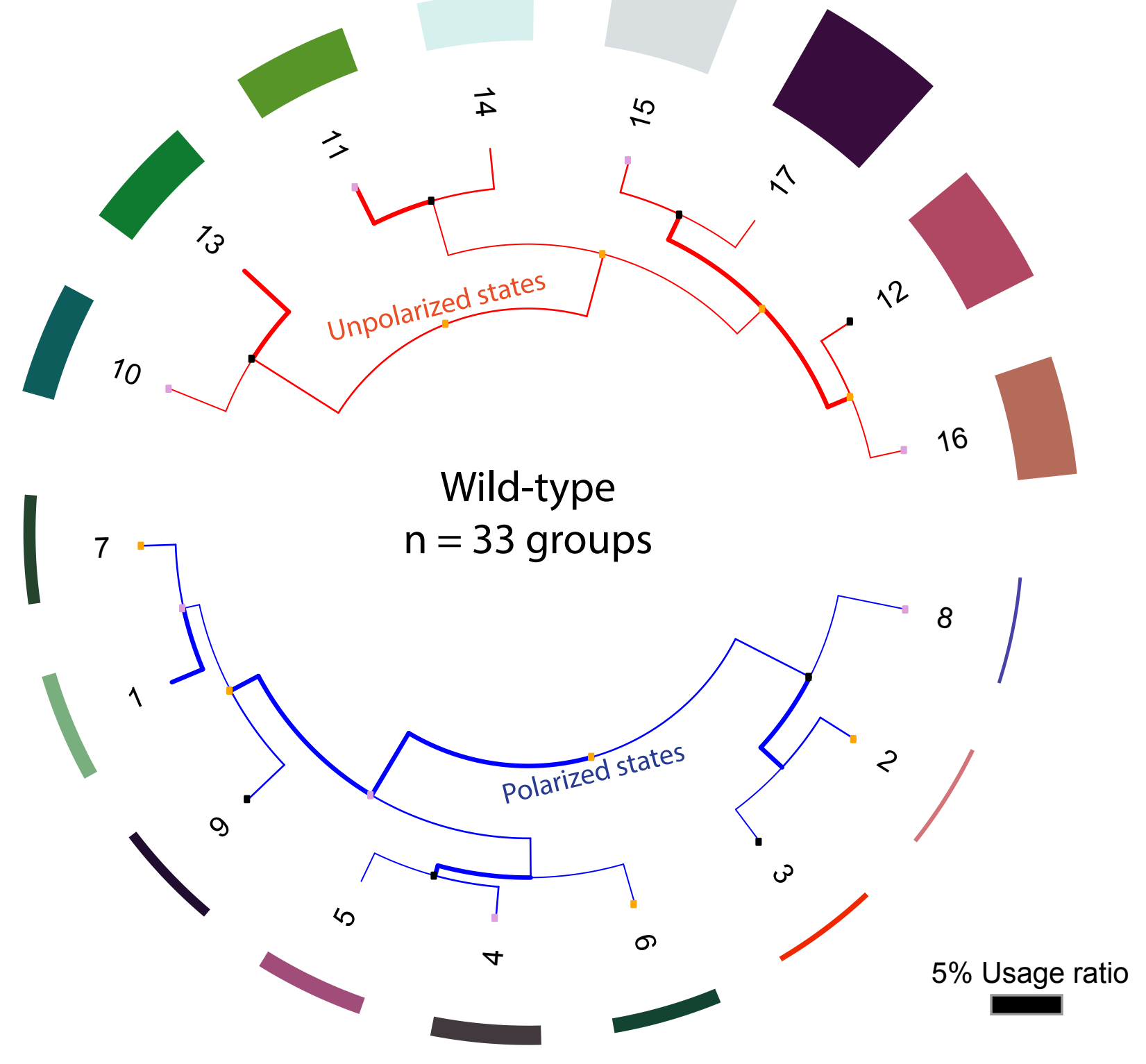

Supplemental Figure 2 Dendrogram of wild-type fish collective behavioral states defined using hierarchical clustering. 


\section{a Polarized states}

immp2I-/-

$\mathrm{n}=9$ groups;

Wild-type;n= 33 groups;

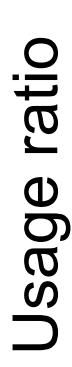

$0.02-$
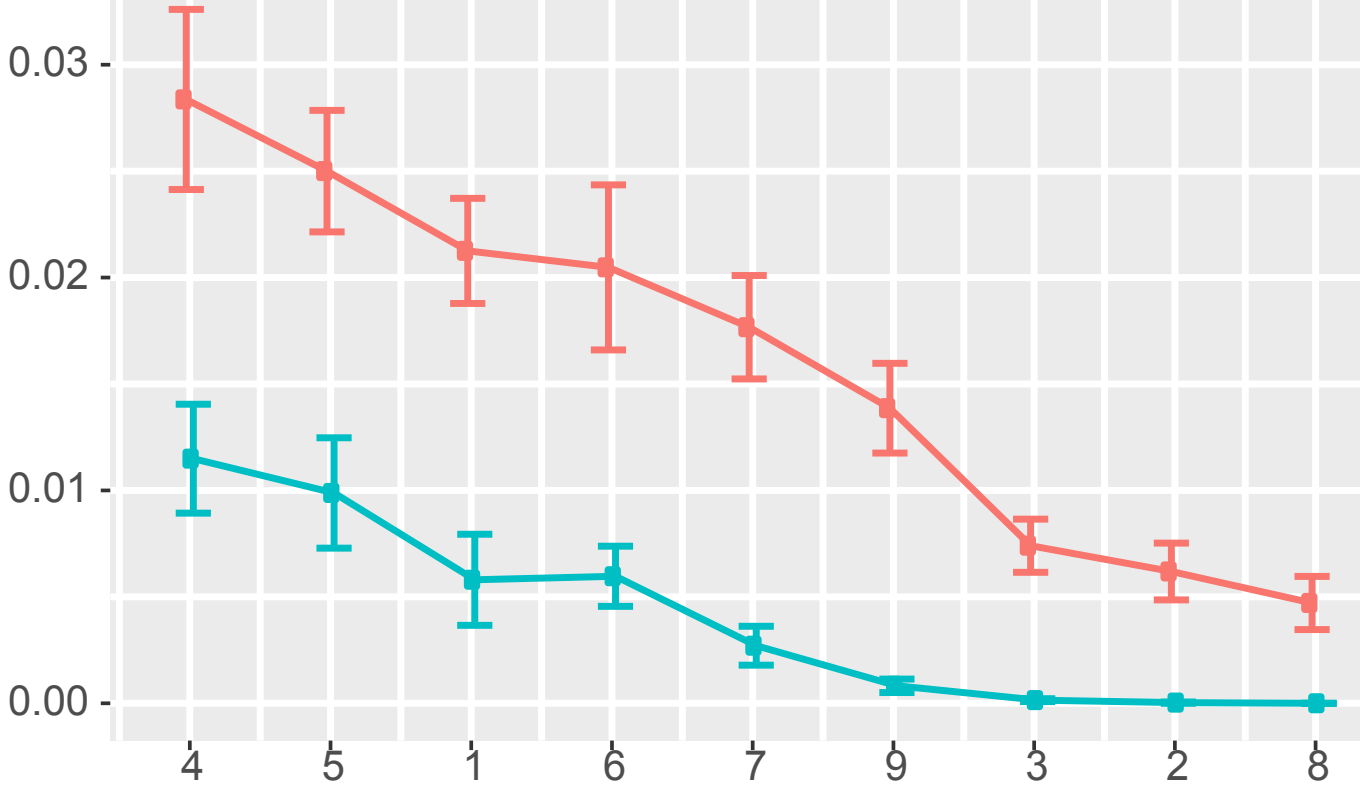

scn1lab+/-

$\mathrm{n}=22$ groups;

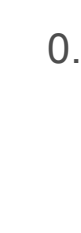

$$
0.02
$$

0.0

0.03 - 0.

$0.01-$

$0.00-$
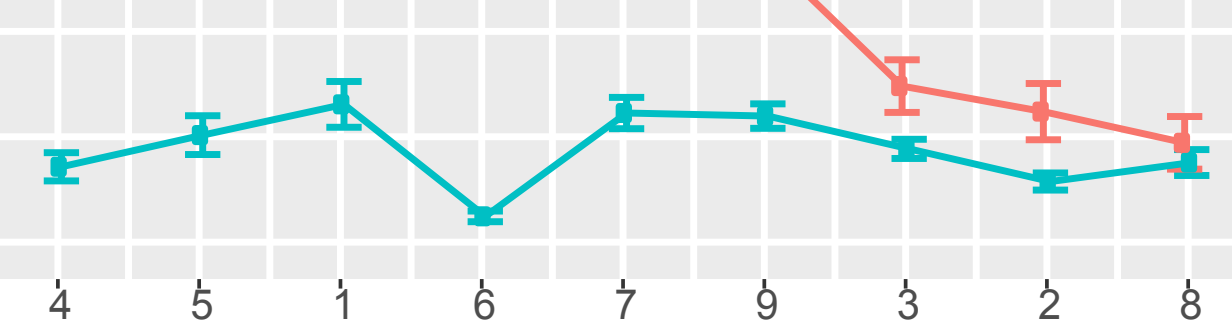

adra1aa-/-

n=9 groups;

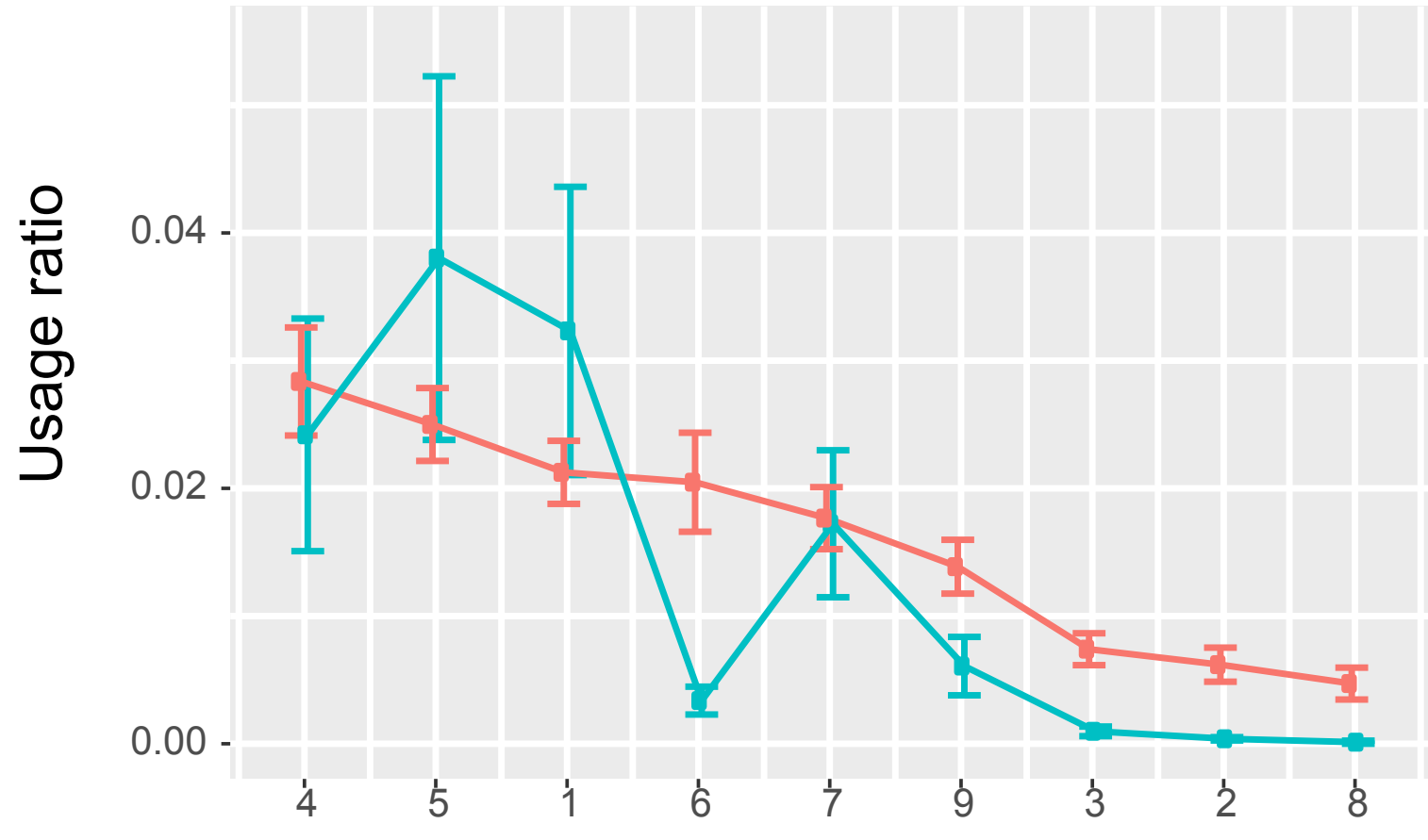

Polarized states (ranked by usage ratio of wt)

\section{b Unpolarized states}
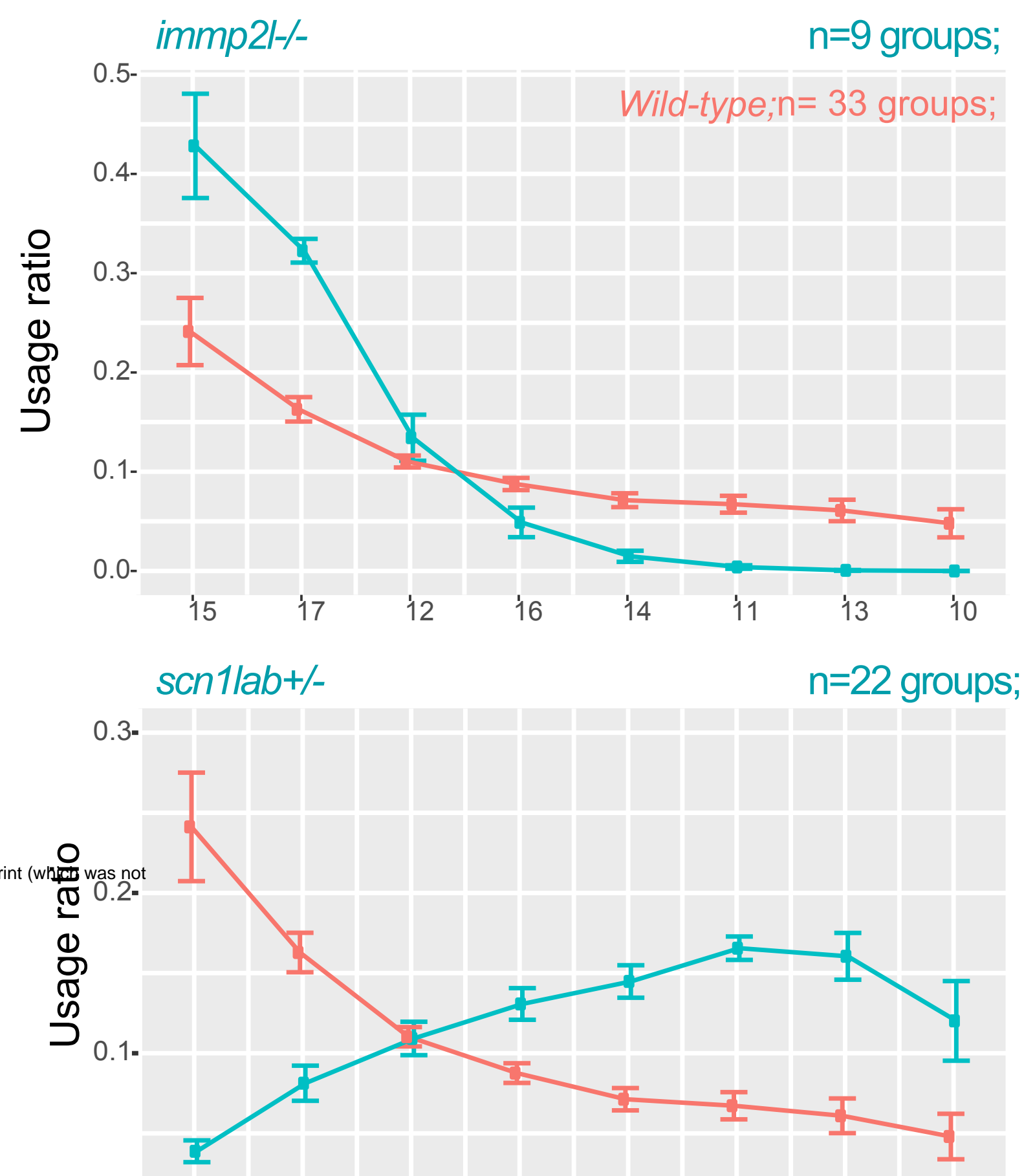

0.0

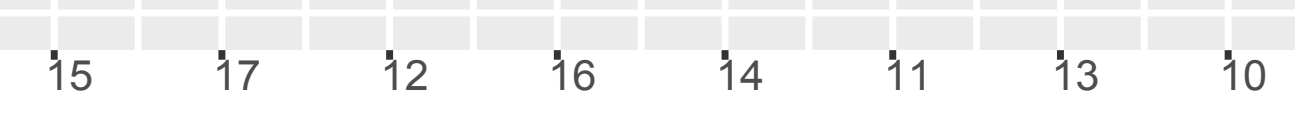

adra1aa-/-

$n=9$ groups;

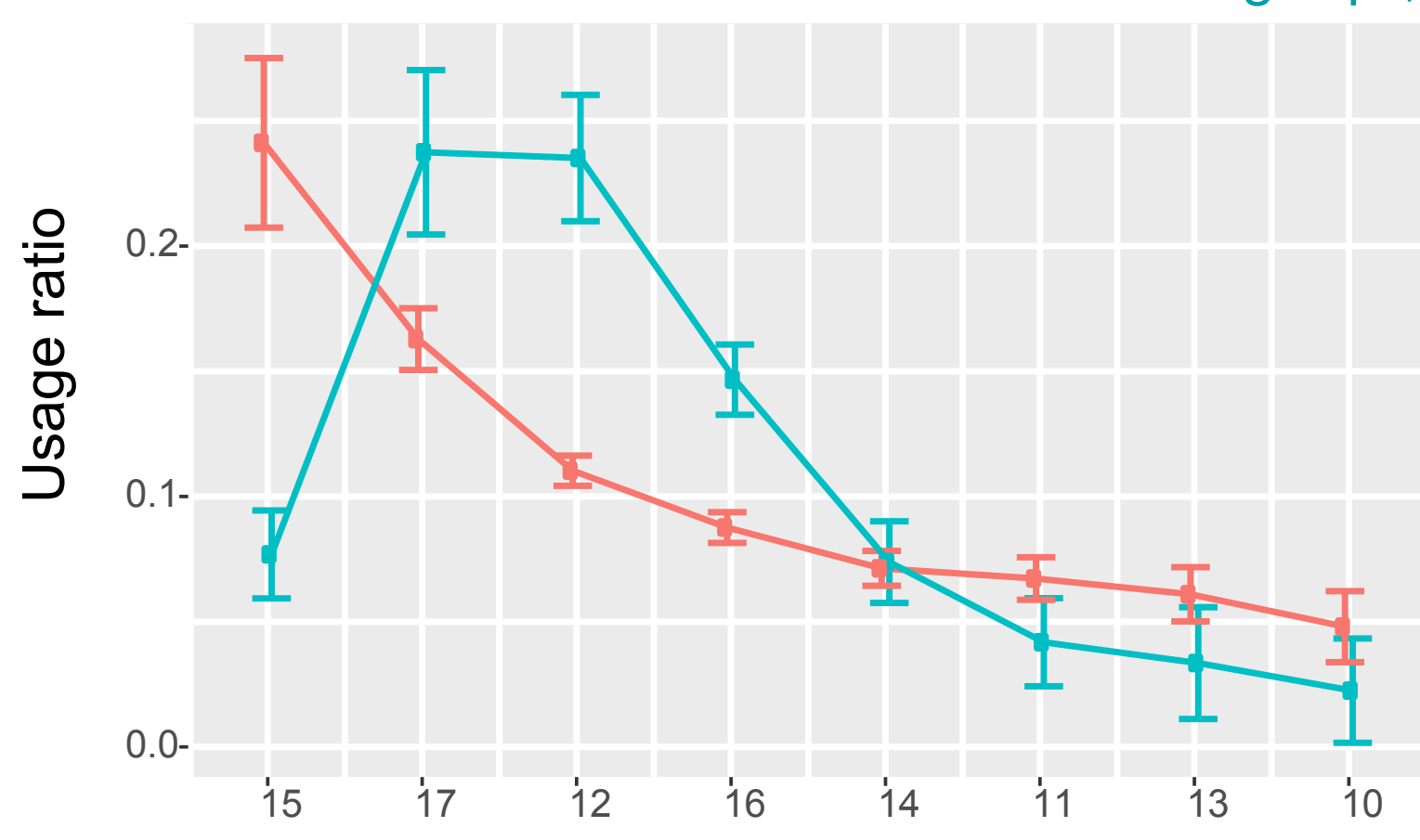

Unpolarized states (ranked by usage ratio of wt)

Supplemental Figure 3 Usage ratios of mutant states. Usage ratios of all (a) polarized and (b) unpolarized states are compared to corresponding states in wild-type (error bars indicate $\pm S E M$ ). The usage ratios are compatible with observations described in the text for the ethograms of Figure 2, and with significance test in Supplemental Figure 6. 


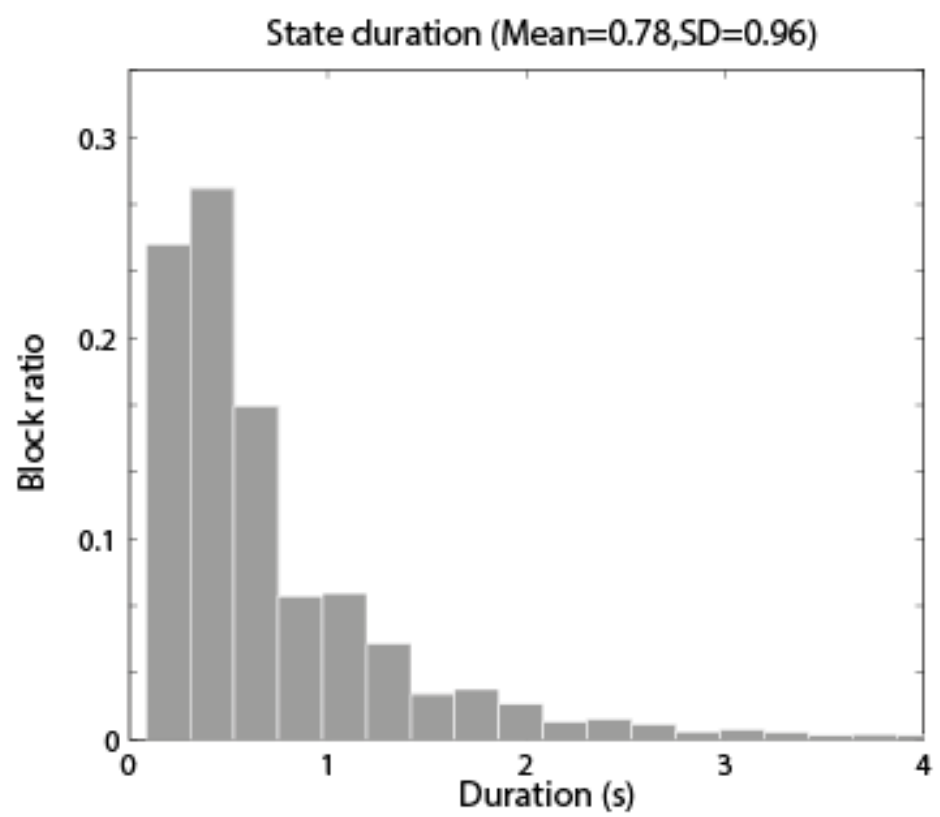

Supplemental Figure 4 Block duration of states (Fewer than $1.2 \%$ last longer than 4 seconds). The mean is $0.78 \pm 0.96$ seconds. 


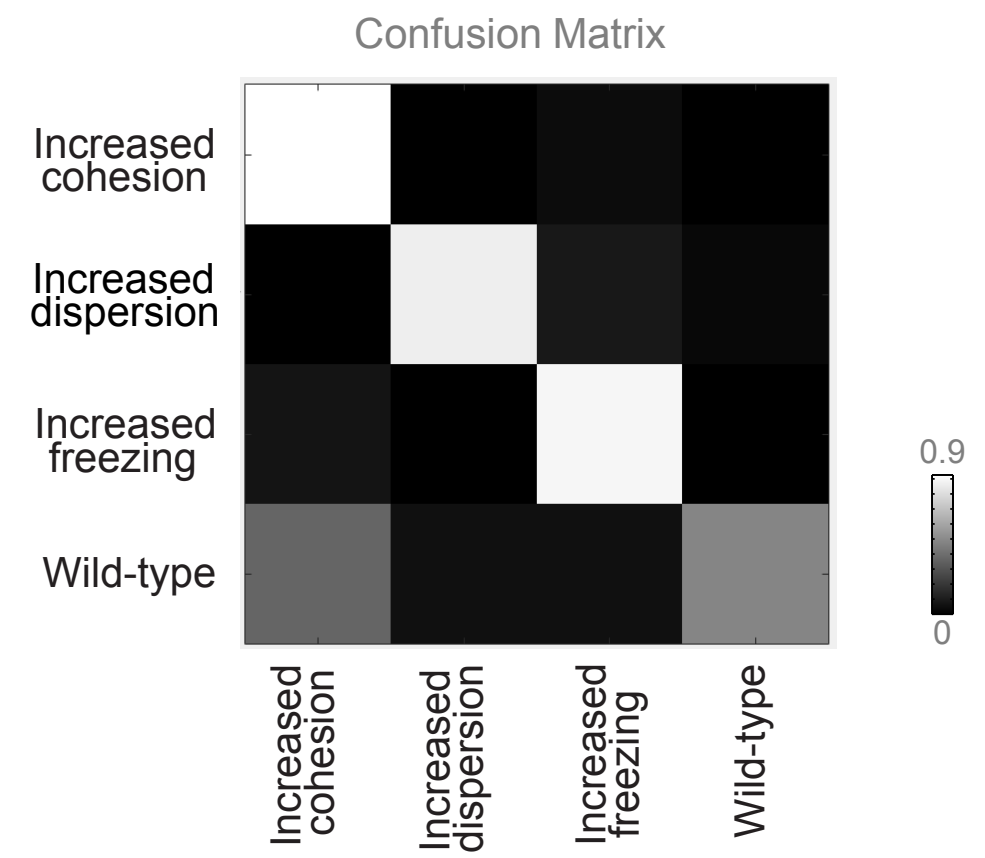

Supplemental Figure 5 Cross-validation by multi-class Support Vector Machines confirms robust phenotypes revealed in highlighted mutants. Confusion matrix computed using features, including state usage ratios, transitions, etc. immp2l-/ $(\mathrm{n}=9$ groups, Prediction Accuracy $(\mathrm{PA})=0.95$; Leave-OneOut); scn1lab+/- $(\mathrm{n}=22$ groups, $\mathrm{PA}=0.88$; Leave-One-Out); adra1aa-/- $(\mathrm{n}=8$ groups, $\mathrm{PA}=0.92$; Leave-One-Out). 

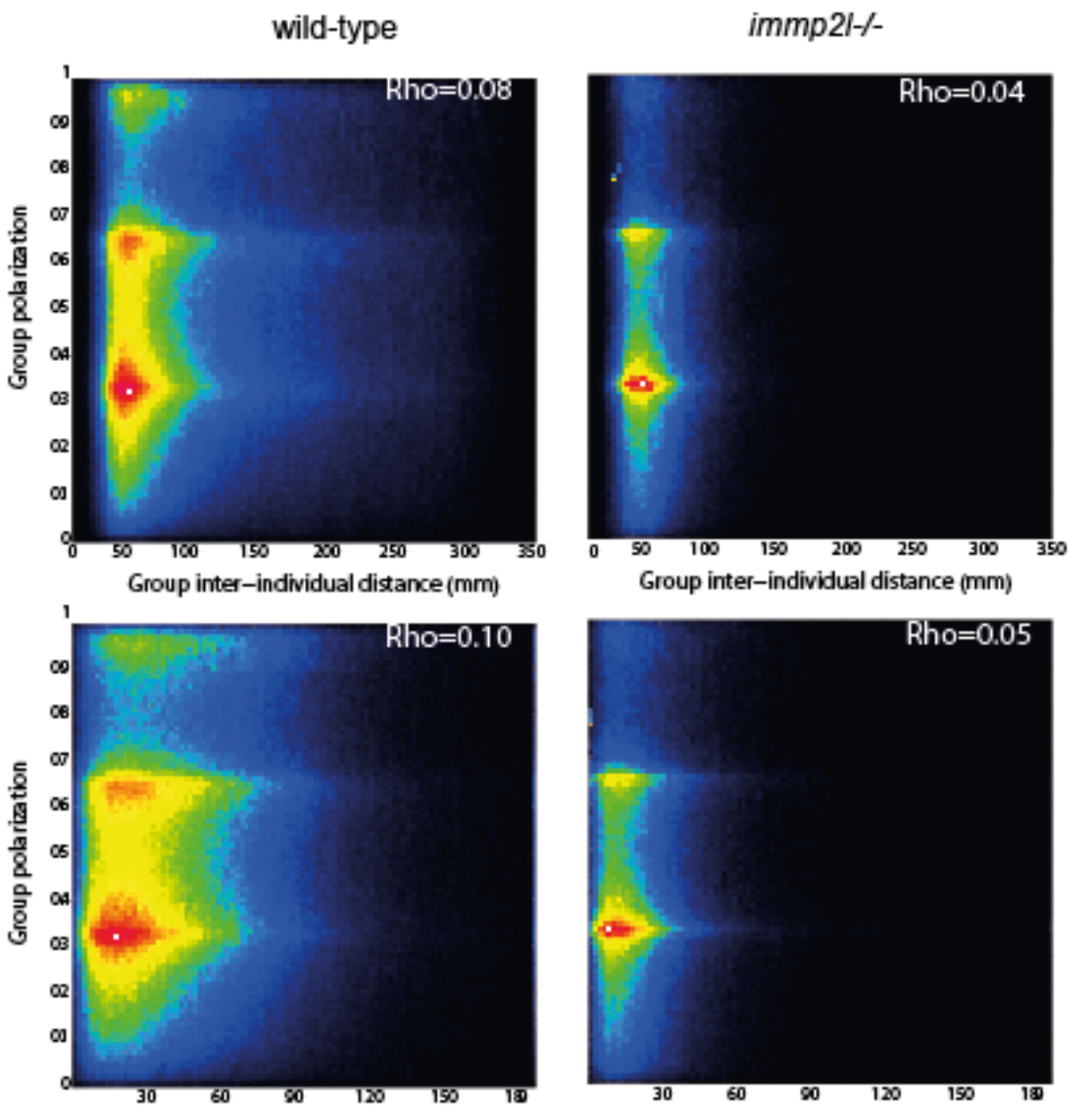

Group inter-individual distance $(\mathrm{mm})$

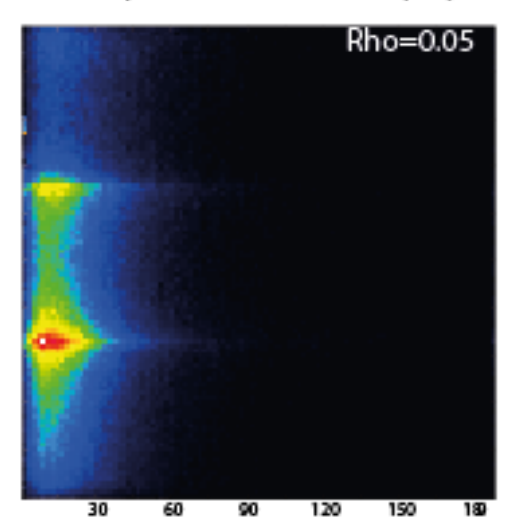

Group speed (mm/sec)
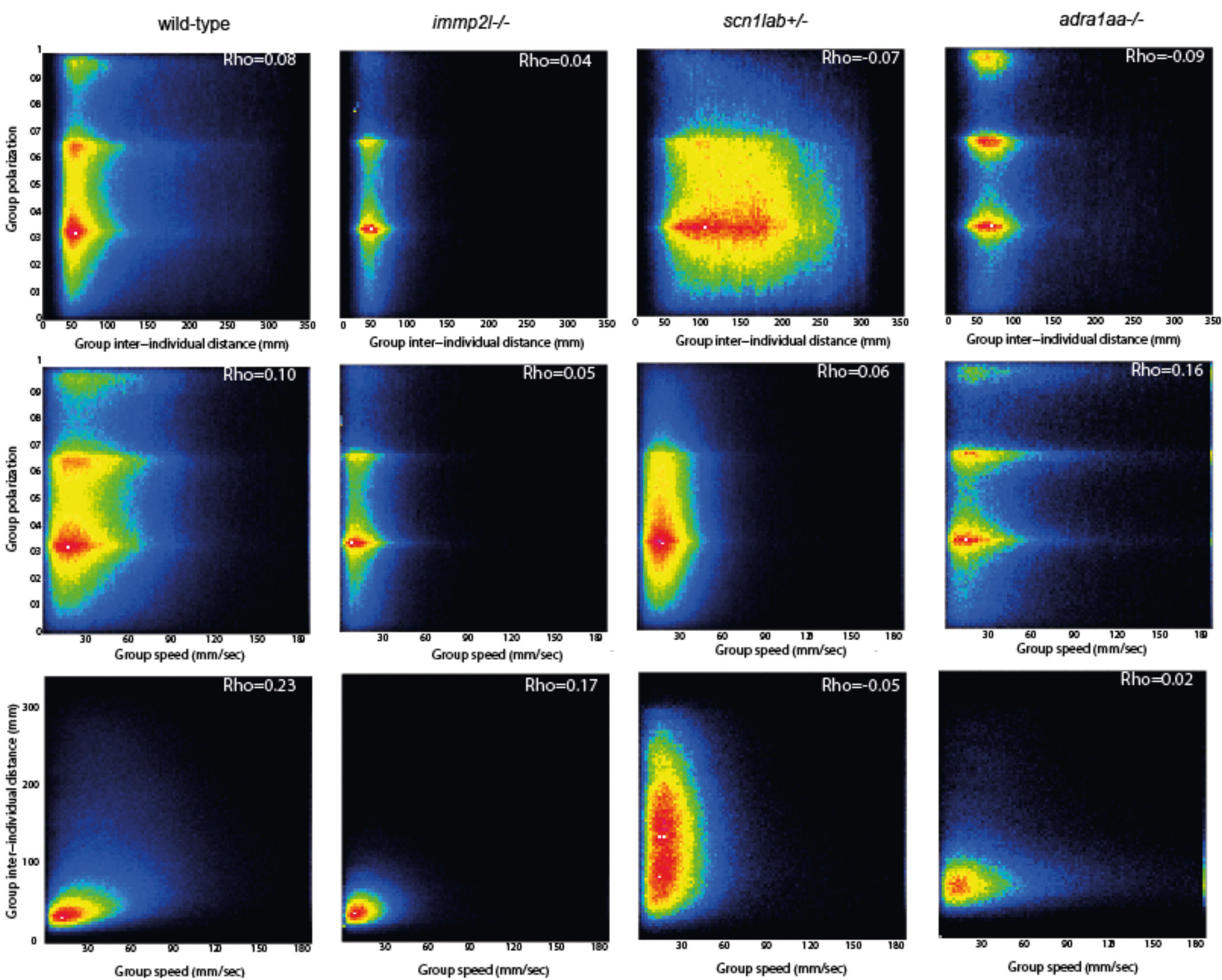

Group inter-individual distance ( $\mathrm{mm}$ )
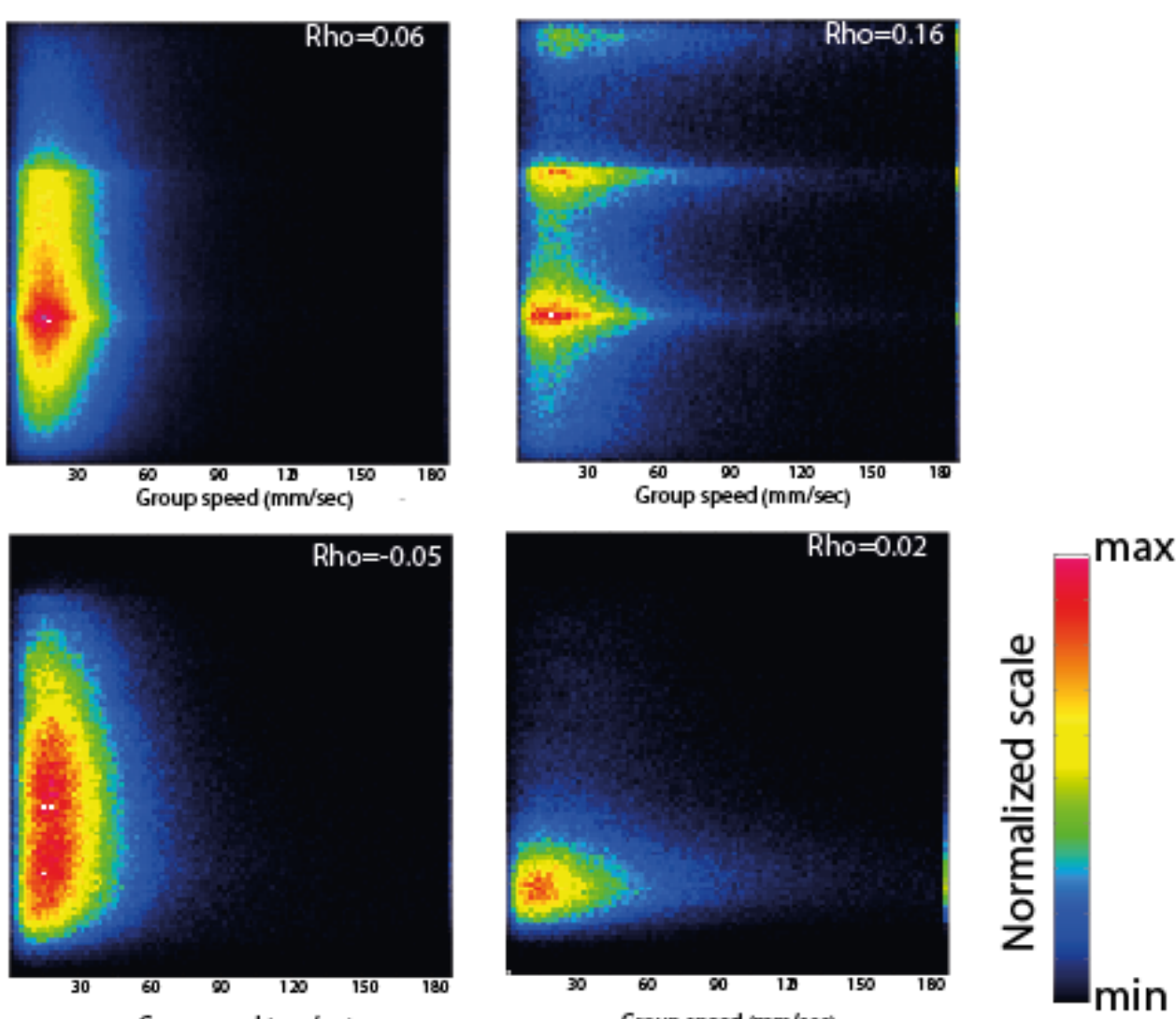

Supplemental Figure 7 Lack of relationship between group polarization and inter-individual distance (top panels) and speed (middle panels) or between inter-individual distance and speed (bottom panels), for the mutations discussed. The colors indicate the number of frames in each bin (100 bins in each dimension). 


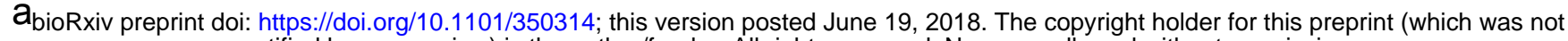
certified by peer review) is the author/funder. All rights reserved. No reuse allowed without permission.

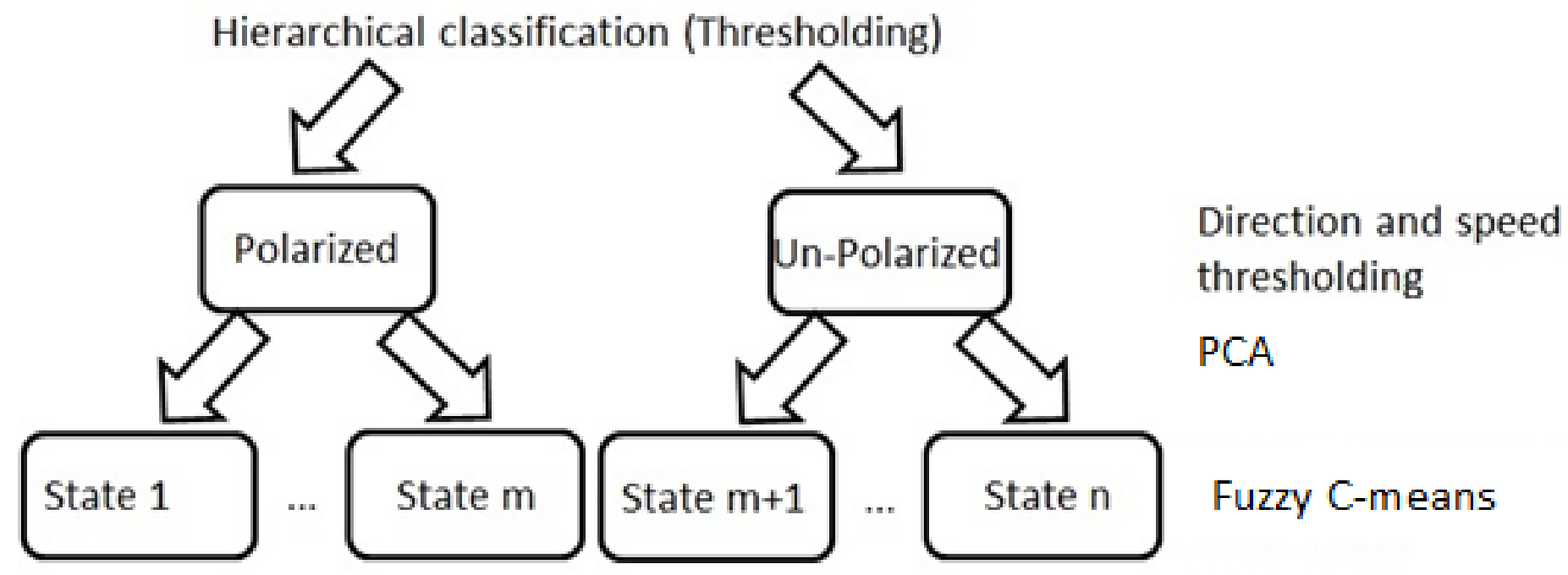

b
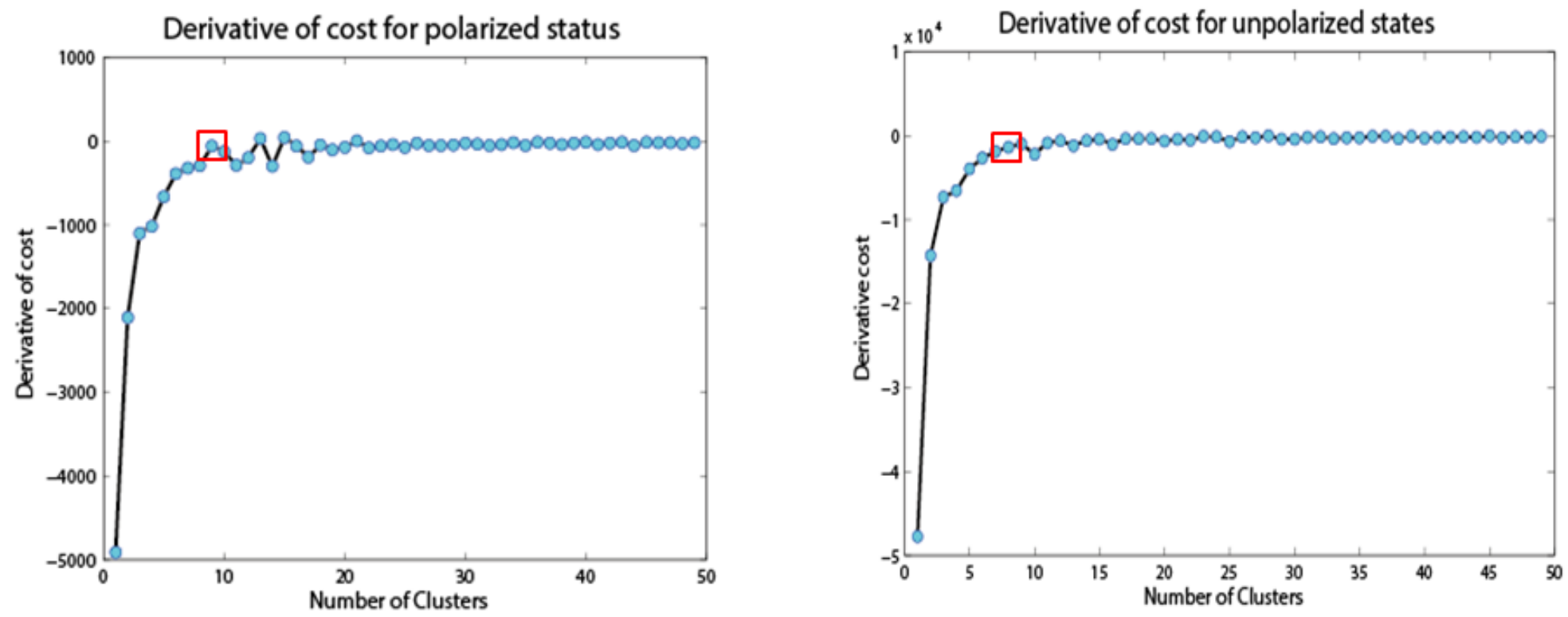

Supplemental Figure 8 (a) Hierarchical clustering flow chart of collective behavior unsupervised learning (b) The derivative of cost of the clustering for porlarized and unporlarized states, respectively. The red square boxed mark the cluster number we chose. 
bioRxiv preprint doi: https://doi.org/10.1101/350314; this version posted June 19, 2018. The copyright holder for preprint (which was not certified by peer review) is the author/funder. All rights reserved. No reuse allowed permission.
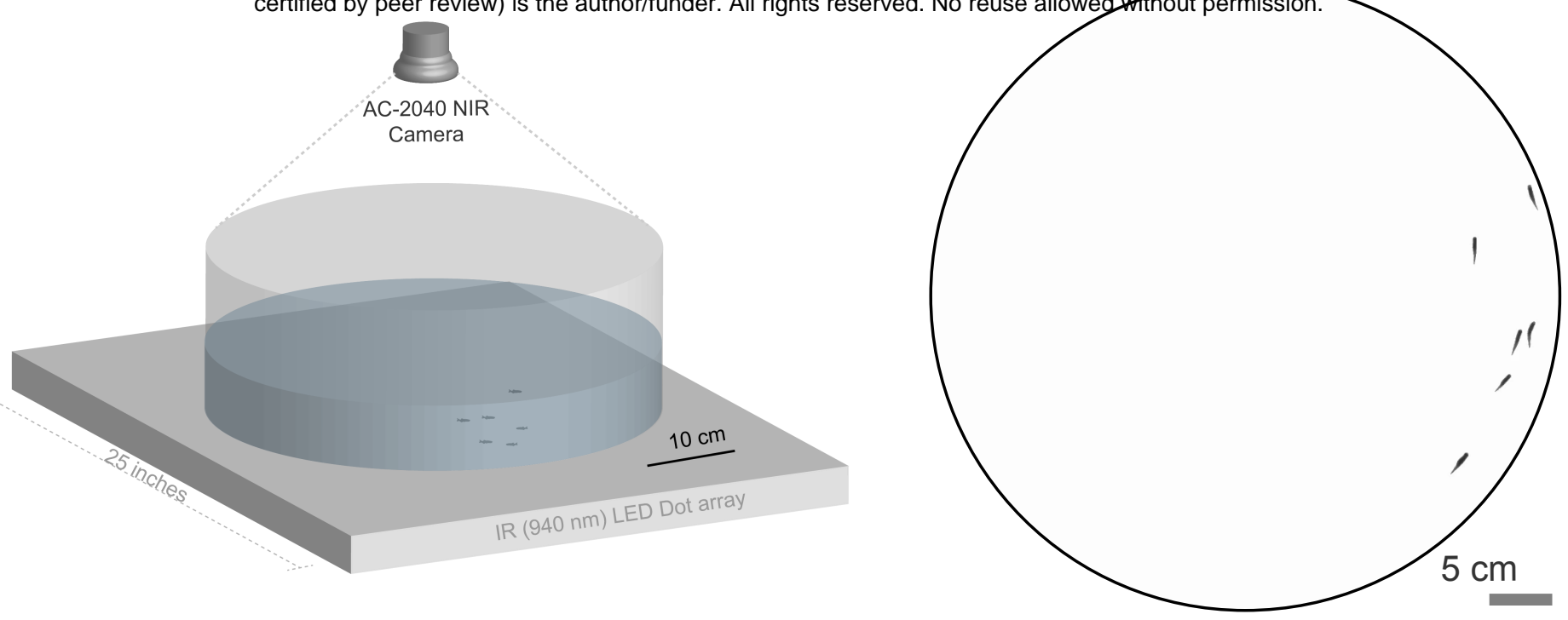

b

d
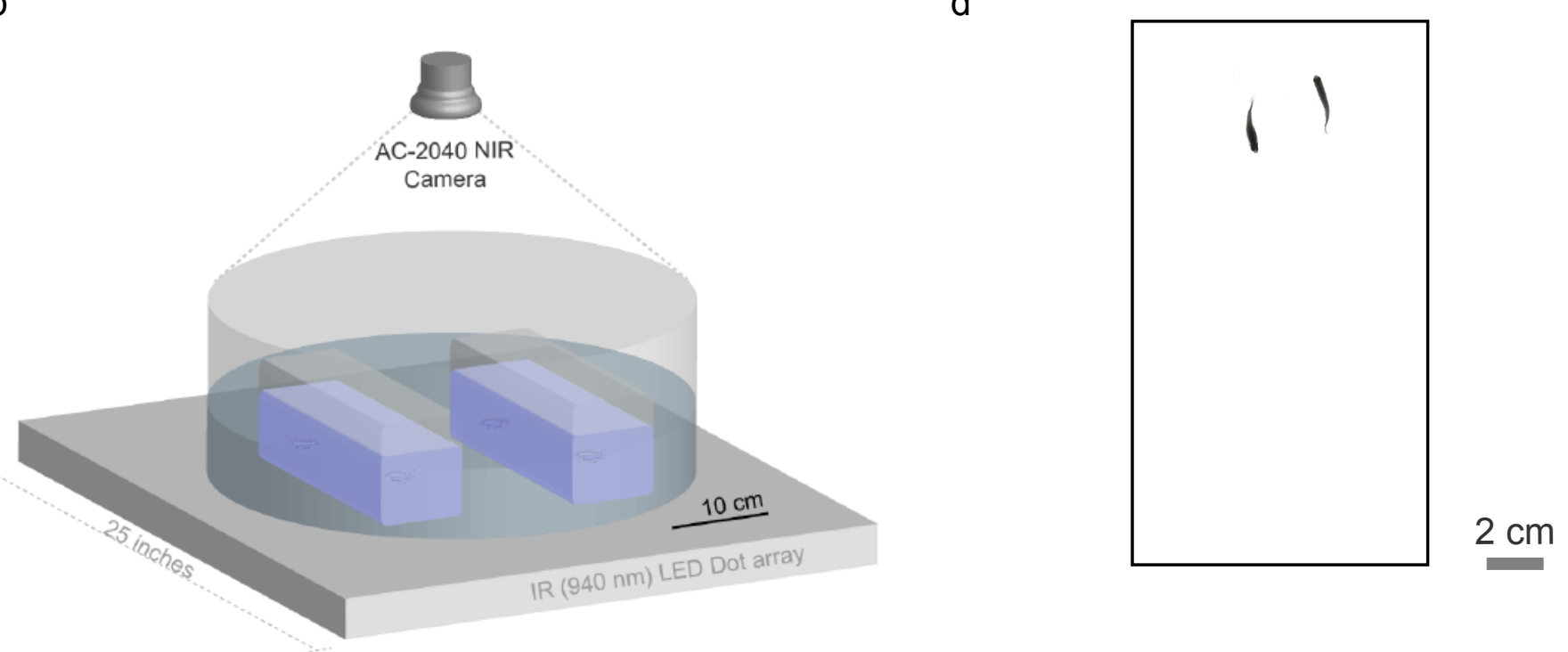

Supplemental Figure 9 Behavioral assay setup (a) Collective behavior assays and (b) courtship behavior assays were recorded from Top View, with bottom IR (940 nm) illumination.

Examples of background removed images are shown for (c) collective behavior assays and (d) courtship behavior assays. 


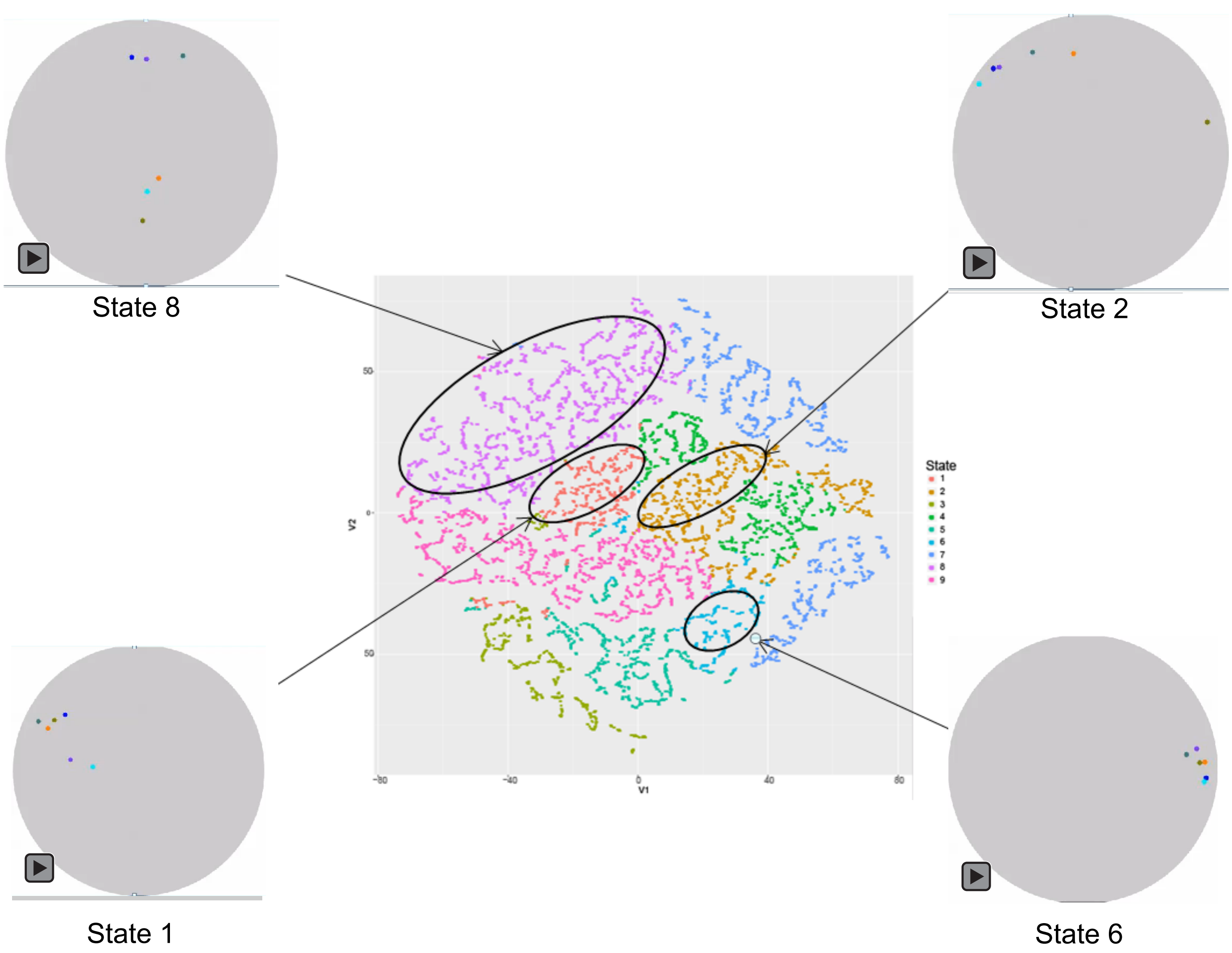

doi: hitps:///doi.org/10.1101/350314; this version posted June 19,2018. The copyright holder for this preprint (which was not
certified by peer review) is the authorf tunder. All rights reserved. No reuse allowed without permission.

t-SNE plot for unpolarized states

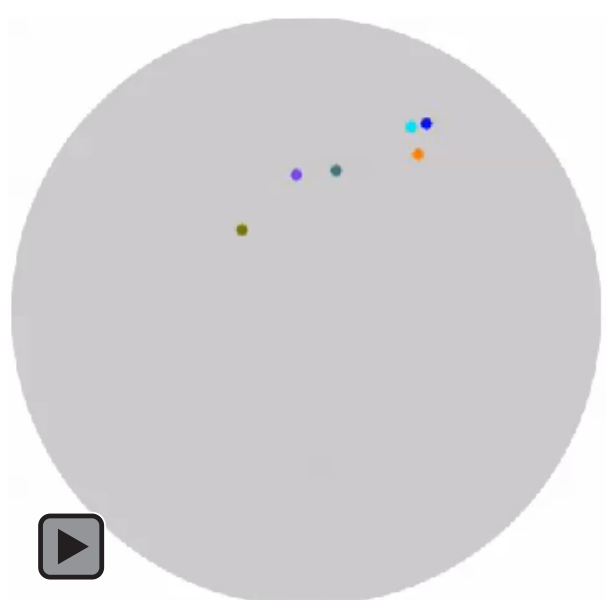

State 16

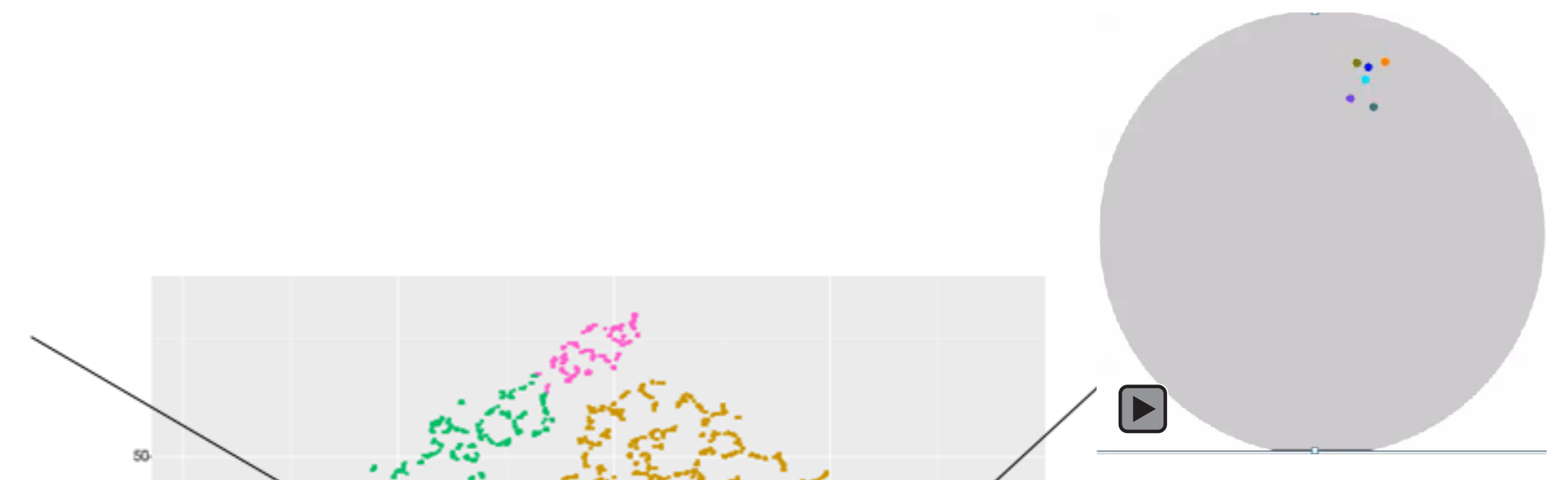

State 15

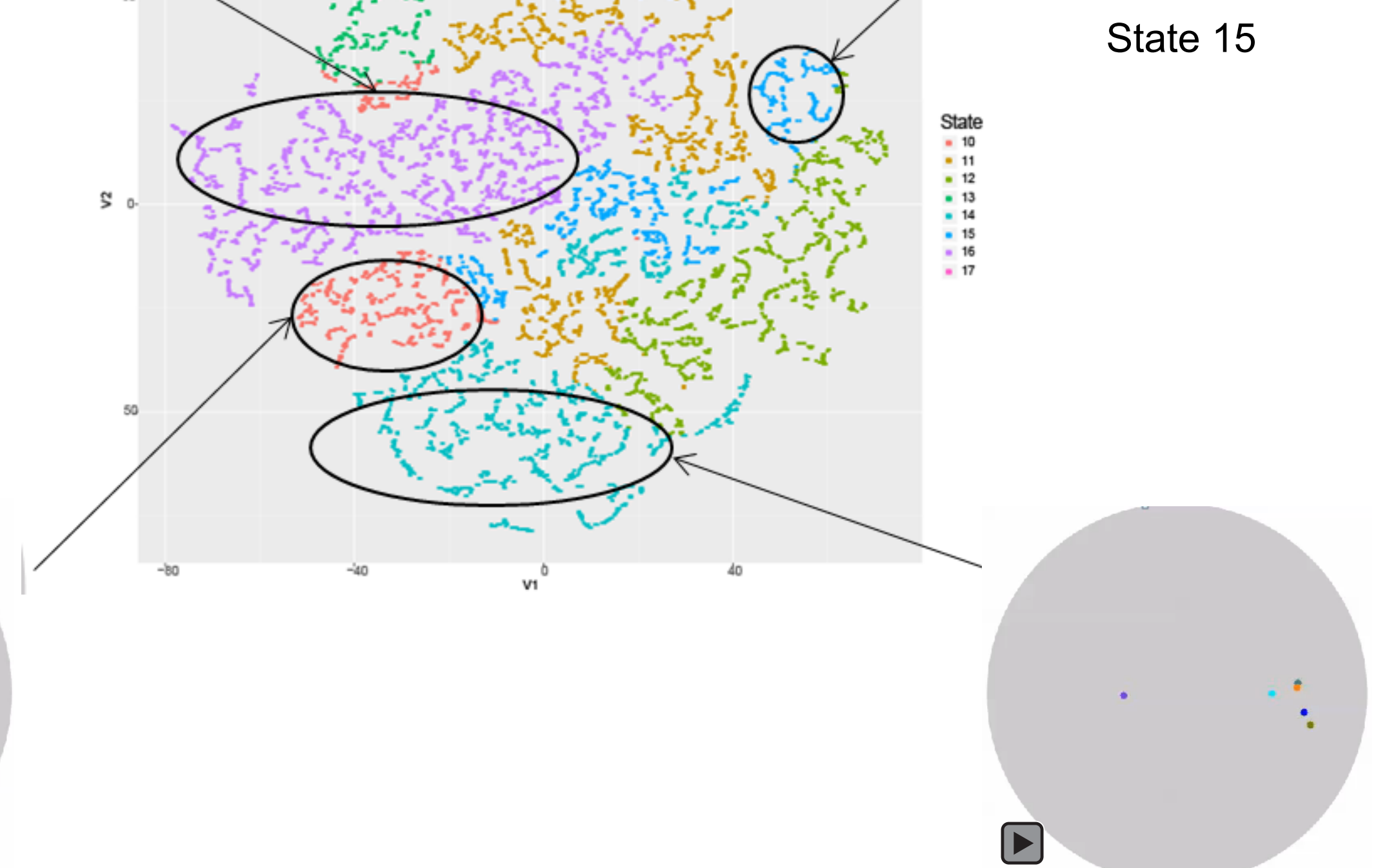

State 14 


\begin{tabular}{|c|c|c|}
\hline Zebrafish gene & Human gene & Description \\
\hline adralaa & ADRA1A & Adrenoceptor Alpha 1A \\
\hline adra1ab & ADRA1A & Adrenoceptor Alpha 1A \\
\hline chd8 & CHD8 & Chromodomain Helicase DNA Binding Protein 8 \\
\hline chrm4a & CHRM4 & Cholinergic Receptor Muscarinic 4 \\
\hline chrna2a & CHRNA2 & Cholinergic Receptor Nicotinic Alpha 2 Subunit \\
\hline $\operatorname{disc1}$ & DISC1 & Disrupted In Schizophrenia 1 \\
\hline dlg $4 a$ & DLG4 & Discs Large MAGUK Scaffold Protein 4 \\
\hline$d r d 1 b$ & DRD1 & Dopamine Receptor D1 \\
\hline$d r d 2 b$ & DRD2 & Dopamine Receptor D2 \\
\hline drd3 & DRD3 & Dopamine Receptor D3 \\
\hline drd4-rs & DRD4 & Dopamine Receptor D4 \\
\hline$d r d 4 a$ & DRD4 & Dopamine Receptor D4 \\
\hline esr2a & ESR2 & Estrogen Receptor 2 \\
\hline gnrhr4 & GPR150 & gonadotropin releasing hormone receptor 4 \\
\hline grm5a & GRM5 & Glutamate Metabotropic Receptor 5 \\
\hline homer1b & HOMER1 & Homer Scaffolding Protein 1 \\
\hline immp2l & IMMP2L & Inner Mitochondrial Membrane Peptidase Subunit 2 \\
\hline kctd13 & KCTD13 & Potassium Channel Tetramerization Domain Containing 1 \\
\hline Irrn3 & LRRN3 & Leucine Rich Repeat Neuronal 3 \\
\hline$n f k b 1$ & NFKB1 & Nuclear Factor Kappa B Subunit 1 \\
\hline oxt & OXT & Oxytocin/Neurophysin I Prepropeptide \\
\hline sapap2(dlgap2a) & $D L G A P 2$ & DLG Associated Protein 2 \\
\hline scnilab & SCN1A & Sodium Voltage-Gated Channel Alpha Subunit 1 \\
\hline shank3b & SHANK3 & SH3 And Multiple Ankyrin Repeat Domains 3 \\
\hline slc18a2 & SLC18A2 & Solute Carrier Family 18 Member A2, Vesicular Monoamir \\
\hline slc1a1 & SLC1A1 & Solute Carrier Family 1 Member 1, Sodium-Dependent GI \\
\hline slc22a15 & SLC22A15 & Solute Carrier Family 22 Member 15, Organic Cation Trar \\
\hline slc25a27 & SLC25A27 & Solute Carrier Family 25 Member 27, Mitochondrial Unco \\
\hline slc39a11 & SLC39A11 & Solute Carrier Family 39 Member 11, Metal Ion Transport \\
\hline slc6a3 & SLC6A3 & Solute Carrier Family 6 Member 3, Neurotransmitter Tran: \\
\hline s/c6a4a & SLC6A4 & Solute Carrier Family 6 Member 4, Neurotransmitter Tran: \\
\hline slc6a8 & SLC6A8 & Solute Carrier Family 6 Member 8 , Neurotransmitter Tran: \\
\hline srr & SRR & Serine Racemase \\
\hline tph2 & TPH2 & Tryptophan Hydroxylase 2 \\
\hline ube3a & UBEЗ $A$ & Ubiquitin Protein Ligase E3A \\
\hline
\end{tabular}




\begin{tabular}{ll}
\hline Associated psychiatric disease & Rererence PubMed ID \\
\hline autism, schizophrenia, ADHD & $19204725,22037178,19918262$ \\
\hline autism, schizophrenia, ADHD & $19204725,22037178,19918262$ \\
\hline autism & $22495309,22495311,24998929$ \\
autism, schizophrenia & 23490763,14606695 \\
\hline autism, epilepsy & 19204725 \\
autism, schizophrenia, asperger syndrome, psy 15386212,17579608 \\
\hline autism, schizophrenia, cerebral degeneration,,$~$ & 25549968 \\
autism, schizophrenia & 18205172 \\
\hline autism, schizophrenia & 22559203 \\
autism, schizophrenia & 25224105,19897343 \\
\hline autism, ADHD & 15892149 \\
autism, ADHD & 15892149 \\
\hline autism, asperger syndrome & 19598235 \\
& \\
\hline autism, fragile X syndrome, epilepsy & 22542183 \\
autism & 22558107 \\
autism and Tourette syndrome & 17043892 \\
autism, schizophrenia & 22596160 \\
autism & 20678249 \\
\hline schizophrenia & $27992301,27759212,22479419$ \\
\hline autism, asperger syndrome & 27242401 \\
\hline autism, schizophrenia & 27271353 \\
\hline autism, epilepsy & 12610651 \\
\hline autism & 26886798 \\
schizophrenia & 28094812 \\
\hline autism, schizophrenia, OCD & 19360657 \\
autism & 22843504 \\
\hline autism & 23116158 \\
autism & 22843504 \\
\hline autism, ADHD, epilepsy & 23979605 \\
\hline autism, schizophrenia & 23583772 \\
\hline autism, epilepsy, dystonia & 16601898 \\
\hline schizophrenia & 19483194 \\
autism, schizophrenia & 15768392 \\
\hline autism, epilepsy & 11543639 \\
\hline & \\
\hline
\end{tabular}



Supplemental Table 2

\begin{tabular}{ll}
\hline Zebrafish gene & guide RNA 1 \\
\hline adra1aa & GGTTCCTTCCAGCCGAATAGAGG \\
adra1ab & GCAGCACAACACGTCAAGAGCGG \\
chd8 & GGATGTTGATGAGGCAAATGG \\
chrm4a & GCCAACTTCAGCTGTGACAGTGG \\
chrna2a & GTTCCAGCAGGAGACTGGAGTGG \\
disc1 & GATTGTTTGTTTCAGTTCTGGG \\
dlg4a & GGATGTGATGCATGAGGATGCGG \\
drd1b & TGGTCAGAATGAGGAGTGAGAGG \\
drd2b & GTCATAATAAGTGTCATTGAGG \\
drd3 & GCTAAACATTGCCATAAGTGTGG \\
drd4-rs & GCCCAGTATAGACCCAACGGGG \\
drd4a & GTCGCTGGTCCACGTGTTTGGG \\
esr2a & GGAAGTGGACTCAGGCCGTGTGG \\
gnrhr4 & TGGCAGCCCACAACACGCCGAGG \\
grm5a & CCATCAGCCGCCAGCAGACAAGG \\
homer1b & AGAAGCAGCCAGAATAGCCAAGG \\
immp2l & AGATCATCAAACGGGTCATCGGG \\
kctd13 & GGGTGGTGTTGGACCGATGCGGG \\
lrrn3 & GGAAAGTCGGGGTTGTTGTGG \\
nfkb1 & GGGGCTTCAGGTTCCGCTATGGG \\
oxt & GGAAAGGCCTGCGGTTATGAGGG \\
sapap2(dlgap2a) & GGAGACTCTCGCTCAGACAGCGG \\
scn1lab & GGTTAATCTCATCCTGGCTGTGG \\
shank3b & AGGAGCCCCGGGCCCAGATGG \\
slc18a2 & GGACGACGAGGCTGCTCAGATGG \\
slc1a1 & AGATGCTGGGGAAGAAAGAGGG \\
slc22a15 & GTCTGGAATGATTTAGAGGAGG \\
slc25a27 & TGGTTTTGGAAGATCCAGGGGG \\
slc39a11 & GCCTGGAAACATGCTGTCTGTGG \\
slc6a3 & GGAGTACTAATTGAGGCCATCGG \\
slc6a4a & GATGATGAATCAAGAGTACGGGG \\
slc6a8 & CGCCCTGAATATGGTCCTGGTGG \\
srr & TGTCGTACCCTATCCAGATGTGG \\
tph2 & GGTGAGAGATATCCAGCCACAGG \\
ube3a & GGACCTGCCACCCACAAGGGAGG \\
\hline & \\
\hline
\end{tabular}




\begin{tabular}{|c|c|}
\hline guide RNA 2 & guide RNA 3 \\
\hline AGAGAATCACCACTAGAGGGAGG & GTATTGTCGTGTGTATGTTGTGG \\
\hline GATCCACAGAGATTACGCAGAGG & GCAAAGGAAGGGTATTGCAGCGG \\
\hline TGGCTCATGTGCAGCGGTTGAGG & CGAGAGCCCGTACAAGACGGTGG \\
\hline GGATGGAGAGGAGATGGATGTGG & AGACAAATGCAGAGAGGTTGCGG \\
\hline TGTCСТCСТCATCATGCAAATGG & TGGCGAGAAATGGCTGACAGAGG \\
\hline GATGCCATGGAAAGCCGCCACGG & GACGCACAGATTCAAGATGGAGG \\
\hline GGCAATGTGTTGGTGTGTATGG & GACAATGAGGTAATTAGTGGTGG \\
\hline TGAGCATTCATTCACCCCTGGGG & GACCACCACCAACTACCTGGTGG \\
\hline AGACCCAACGGCGGCCCACGAGG & AGCGTTCGGTCAGCACACTGAGG \\
\hline GGCTAAGGCCAGGATCCACGTGG & TGTTGATGCCGAACATCACGGGG \\
\hline AGAGGATGAGTTGAAGATGGGGG & AGGGCGATGGGATGCAGATGGGG \\
\hline CGGCGCCACACTTGCGCTCGTGG & GCAGTATGGCATCCAGCGGGTGG \\
\hline GTCTCTAGAAAAGATGGAGCTGG & \\
\hline GGGTGTGAGGGCCCGTCTCACGG & GGACTTGCGGTTCTTCTCGCTGG \\
\hline GGTGCTCGATGGAGAAAGAGGGG & GCTGAAGCCTGTCACACGTGTGG \\
\hline GCGGAGAAGCAGAGATGTGAAGG & AGGGTTTGCTGAAGAGGAACTGG \\
\hline GAGGAGGCTTTCCAGGTGGTTGG & TGCATGTATCATTAGTTACCTGG \\
\hline GGCAGATCTGGAAAGAATGGTGG & GCCCTTGAAACTGTGGCAAGGGG \\
\hline GGGCCTGAACCAGCGGACTGAGG & GGAGAAGATGAAGACCAGTGCGG \\
\hline AGAGAACGGCAGGCTGGTTGTGG & TGGAACATTTAGGACTCTGGTGG \\
\hline GTCAACGGGACCCTCAACGGGGG & TGGGTTTTGCAGTGGGGCTGGGG \\
\hline CGTACCCTATCCAGATGTGGTGG & GATGTGGTGGTTGTTTGCTGTGG \\
\hline
\end{tabular}




\begin{tabular}{ll}
\hline guide RNA 4 & guide RNA 5 \\
\hline GAAACGCGAGGACTGATCAGCGG & CGCCAAGACTTTGGGTATCGTGG \\
GGTTCCCTCCATCCAATAGTGG & GTACTGTAGGGTGATGTAGTG \\
GCCCAGTGGTGTGTGACCTGTGG & GAAGGACAGGATCCAGGCAGAGG \\
\hline TGAGTCAGACAAATGCAGAGAGG & GCTCCAGCATTTGGGACATGTGG \\
GTTCTCCACAGCAGGCGACTTGG & GTCTGAGCCGATTAACAGCTGG \\
GCGAAAGATGACACCCAAGGTGG & \\
TGGCCACATTAGTTATGCCCTGG & \\
TGCCCAAGGCATGACCAGTGAGG & \\
GCCGCTTTATGTCTACTCTGAGG & \\
GGGCCTGAGGAACTGGGAAGAGG & \\
AGGGCAGAGTGCTGAAGTCGTGG & TGTGCTGTACCCCAGCAGAGCGG \\
\hline & \\
\hline
\end{tabular}

GGACAGTCGGACTGGTTTTGAGG 


\begin{tabular}{ll}
\hline PCR primer $F$ & PCR primer $R$ \\
\hline TATGAGCTTGTGCGTGATTTCT & TCCCAACATTGACAGGTATTGA \\
\hline ATCCTCCTTCCTGGTGTCATT & CCTTCGATCCGTGACTCATTAT \\
\hline TGTTCTACTGTACGTTGCCAGC & CAGGCCGAGCTGATGGAAGAGC \\
\hline GGTTGGAAAGGACTGGATGTAAC & TCATAATGAATGTCATGCTGTTTCC \\
AAAGTCTTCATCCTCAGCCCT & TCCATTGTCATTACCGTTTTG \\
\hline TGCATTTCCTCATTTGCAAGTTGG & GCTTGATTTTGATTGGCTTACC \\
CTATGACGATTGTTTCAGCCCCAA & TGCAAGATCCAGCAGTTCTAGAAA \\
AGGAGGAGATGGCGTATGTC & CAGCATAACTACGGACAAACCA \\
TATTTGCATACTCACAGGCTGGG & AATGACAACTCAAGATATGATGG \\
CTCAAGTCACCAAGCAAGCA & TTGCAACATAGGTCAAATGGTC \\
TGAGGGCGCTTGTTCATATT & GCTGCCATGAATCCATCTTT \\
AAGGGGCAAACTGAAGGACT & CCAACATGGACACAGGATCA \\
AAGGCTTGTGAAGGGTGAGTAAAC & GAAGTTCACCATGACACACATCAAC \\
CCAGAGGGTTGAGAATGGCCGAC & CCTCACTGTACAGCAGGGTGTCG \\
GGTTTTCATTTGCTGGTGTTT & GGATGGCCACAGAGCTGGAT \\
CACAAAAAGAGAGTCAGAGGCAAATAC & ATTTGCAAAGGTAATCAGGGTGTT \\
CCTCCAATAGATAAGACCCATCTGG & TTTCAAGCTTTATTTTTATGGTGA \\
TGTAAATCCACCAATCATGCTTCTT & AAACATAGTAAGTCTAAGGGTACACGCC \\
TCAAGCTGCTCTCAGACAAGTGC & CCATTTCTCGTGAATGCACCTGC \\
CCGAGTTCAGAATACACGTTCAC & CCATATCAGTGCATCCCTAGCAT \\
AGGAACGGTCATAATAGGTTTCCC & TATCTAGCGCCTTTACAAAGTTGG \\
ATGCACTACAGTTCACATCAGGAC & AGTGAGTGATATGATCTCCGTGGT \\
AAACTCTTCCTCTTTCTGTTGAGC & TAGGCAAGTCTTCATCACTGACTC \\
TTTGTCTTCAGAGAGCACAGACTC & TATGGAAAGGCAGTTTAACCAGAT \\
TTTTCAGCACGGTGGTTATG & TTAAACTAGCGGCTGTACCTG \\
CCTAAAGATGGGCTATGTCTGAA & GGGATTTTACAGTGTCATACTGG \\
CAGTCTCATCCAAACAACTTCG & AAGACAACGTTTGACCCTCAAT \\
CTGGCCATGACTCACCATTAT & AAGTTCACTTTATCAGCCTGTGC \\
TGACACCAAATACTCCTCAAAA & CTGTGGTGTTTCATTGTCCTGT \\
CACTGTAATGATGGAGACGAGTGG & ACTTGGGGAAATGTCATCGTAGG \\
ACTCTGTTCCACCCCTTAACC & ATTTGACCTTACCCCCTCCAT \\
GTTCTCCACCGTGTCTGTGAC & AGATCCCTCGCACCTGAAGA \\
TACCTCCTTCTGGTTCAACTCC & TTACTCTGACTGTGCTGGTGCT \\
CAGAAGTGGTGAAGGACAATGACA & ACAGGTGTAAAGCCAAGTCAAACT \\
ATGTCTGATACAGGAAGCGAGCG & CTGCTGGCCTCACTAACACCACT \\
\hline &
\end{tabular}


Reference sequence

TATGAGCTTGTGCGTGATTTCTGTGGACCGATATATCGGCGTTAGCTATCCTCTACAr ATCCTCCTTCCTGGTGTCATTCTGGGCTTTTCGACAGTGGATACGTAGAGTCATTCCI TGTTCTACTGTACGTTGCCAGCAAAAGTGTGTGTGGATGTTTGTTTTGTGCTATAACC GGTTGGAAAGGAACTGGATGTAACATTCCCCAGGTGCTACGGTTCGGGCTCCGACG AAAGTCTTCATCCTCAGCCCTCAGATGATCAGCGATGTACCGCACCCCTTCCAGAGC TGCATTTCCTCATTTGCAAGTTGGTTTCATTTCTCAACAGCTGTATTGCACGGATCAC CTATGACGATTGTTTCAGCCCCAACTTCAGACACGCTTTCCGTCAAGCATTGACGCTI AGGAGGAGATGGCGTATGTCCGATTAAGGCTGGAGTCGCAGTTATCTGGGGGAAGT TATTTGCATACTCACAGGCTGGGGTTGGGGTGTTGCTCAATGCTGTGTTCTTTTCTT1 CTCAAGTCACCAAGCAAGCAGCATGTCATCTTGAGCAGTGAATTGTTTACCGAGAAT TGAGGGCGCTTGTTCATATTTATGGCTTCATCTTGTCATAATTCCTCTTCTTGTAACA ${ }^{-}$ AAGGGGCAAACTGAAGGACTACCATAAAATATGGGTACAAACAGTTTAAATTCATGT 1 AAGGCTTGTGAAGGGTGAGTAAACAGTGGCAGAAATTAAATTATGTGAACTATTCCT' CCAGAGGGTTGAGAATGGCCGACTGTCTGTCTAGGCTTATGACCACGGTCACAAATC GGTTTTTCATTTGCTGGTGTTTGTGCTGATTGGGAACAGATTGGGTCCTACCCGGAT CACAAAAAGAGAGTCAGAGGCAAATACTTTTTCACAACAATGCATCATTTGTCAGCTC CCTCCAATAGATAAGACCCATCTGGTCTCTGAAAACTGTCCCTAATGCCCCATAATCC TGTAAATCCACCAATCATGCTTCTTAATTTGATGTAGCGGTTAGCTAACTGAACTAAC. TCAAGCTGCTCTCAGACAAGTGCGGAACCTCAAATTTCTGGACCTTAACAAGAATCC, CCGAGTTCAGAATACACGTTCACACTAGCTAAACGTACCATACTATGACGTCAAACG, AGGAACGGTCATAATAGGTTTCCCTTACCATCTGCAGTGTATGCCGTGTGGCCCCGC ATGCACTACAGTTCACATCAGGACAGCTTGCGGGACGACGGCTCCACTTCTCATGG AAACTCTTCCTCTTTCTGTTGAGCCTCCTCTATAGTGGCCTGGTTCTGCTCATCATAC TTTGTCTTCAGAGAGCACAGACTCCGCTCATGCAAGCAAACCACGTTGTGGGGTGG( TTTTCAGCACGGTGGTTATGTTGTTTCCTCTATTTCATCTCTCTAGTGCCTATTATCCC CCTAAAGATGGGCTATGTCTGAATTCAAACACACTAAAATGTGTTGTATTAACCCAAC CAGTCTCATCCAAACAACTTCGTCCTTTGTATCACGTGGTTTCATTCCACGTCTGCGF CTGGCCATGACTCACCATTATAACAGTATTCATACTGAATAATTATTACTAATTTAGCT TGACACCAAATACTCCTCAAAATATCTTCTTAATTCCTCTAAAGAAACGAGCAGATTTC CACTGTAATGATGGAGACGAGTGGCTGGTCACACTGTCTGAAAACACATCAGTCTCT ACTCTGTTCCACCCCTTAACCCCCTTCTCCAGGCTCTCTATCCCCTCATGGGCACGA AGATCCCTCGCACCTGAAGAGGAACAAAAAGGGACATCTTGGTGTGAAAAAAAACTC TACCTCCTTCTGGTTCAACTCCATAGATCTTTGTGTCTTCACAACCAGACAGTTTGAT CAGAAGTGGTGAAGGACAATGACAGGATTGTGTGTGTGCTAGTACTCACGGCTCTG। ATGTCTGATACAGGAAGCGAGCGGCTGCAAAGCATTTAATTGAGCGCTACTACCACC 
ЭTACCCATCTATTGTGACAGAGCGACGTGCATTGCTTGCGCTCATAGCTTTATGGG: こTGCTCACTTAACCCATTCGTCTCCCTGCTCTTACTCATTGCACGAGTCTTGTGTCG ¿TTTTACATCACATTTTATTTCAACTGCACACAAGCAACAAAAGCACAGATTCTGAAT ¡ATAAACTGCCAGAACAGGATAGCGGGAGCCCACAGGATGAAGGACAGGATCCAGC :TCGCAGGACACACGGTGAAAACAGCAGACCAGGCTCTGACACCAGGTTACCAAGC ACTAATTTTATTGCTCAGTCACATGAATGCAGCTGTCATGGGTCATTATTTGTTTAC, GAAGCCCCGTGTGAATGGGGCGTTAGACCAAAAAGCGTGGAGATGAATGTGTGCA TCGCCATAGGTACCGTTAAGCTCTGTATAACTAGTCGTCTGGGCTTTGTGCCAATT ГTGTTTGCATGTTTTTGTAAGCTTTTGTGCATATCAGAATATTTATTCTGGTATTTGG( TTTGCAGAATATAAAATTCAGAGATGTCAGTAGACAAACACAAGGATTCAAACAGAT ГATGGGCAAGAACACAAACACGTATTAGCATATATTACACACACAAAGAGCCATCCA ГAAATTTCAGTCTTTGTTACAGTAAAATATTAATAATAATAAATCACCTCATCTTTTTG ITAAGAACATTCCATATGGAGTATTAAGCTTAAACCTACCTGTTTTCCTCTTCAACAT ICACAGGAGTACATAGCCACGAGCTTCAGAAACATTAACAATCTGCAAGCCAGATC' TTTAGCCGAGGCCCAGGCTAGTGAGCGACGAGTTTTGGCCCACATTCCTGGAGAC ;CAAAAATTACAATGGACGCGAATCACATTTAATCTTTTCCTCTTCTCAGTTCGCAGA ¿ACTGAGCTCAGCAGCATCCTCAAATAAACCGCTAGCAGGTCTGTCATCACTATGC/ AGATGCAGAAACTAGCTCAGAGATGCACATTTAAACATGTGTCCATTTGTATTGAAC CATCGAGAGGATCCAAAGGGGTGACTTTGTCGACATGATCCATTTGAAAGAACTTG AACCCGGGTGCGGACCAAAAGTGCTAGTATGAAAGCACCCTAAATGAATGAACAAA ¡GGACCGCGGACGCTGTTTCGGCCCCAGTATCTGCTGTGGTGAAGGCATCGGCTG ГGGAGGAGGTGGCGGAGGAGGGGTTAGCATTGTTAGCGGGGGTGGCGGATCCGG :GCCATGGCCACCACAGCCAGGATGAGATTAACCAGATAGAAGGAGCCCAGGAAG/ उGCGTGGTCGCCCCGCCCATCTCAGGGACCCACTGCTTAAACAGTCATCAGACAG ¿AAGTTACCTGTACACGGTGGACGACGAGGCTGCTCAGATGGTTAAGAATCACTCC :AATGAGTCAATAATGGGCAATAATTACAATTCTTTTCACCCAACTGTTGGGTTTATC \AGGGAAGCCAGATTTACAGCGGAGCATCTGAAACCTCGACTGCTATCAAACCCCA TTTTTTTGCACATGCAGCTAAAGATTAAGTTAATTTAGATTTTGGTTTGTGCATTGA7 כAGTGAACATTCTCATTTCTCAGAGTCTCTGCATGATATTAATGTGCATCAGCACAG AACGCAGCATCAGCTTGCTGGAGAAAATGCTGCAGCTCACCGTAAAACCAGGCGA TATCAGTCAGTGCAAGAGGACCACCATCTCAACCCTAACAGCAGTCCTCACCATGG ¿ACTTAATCCAAAAACAATAAGAGATCAAATATTTATTTTTGACTGCTGATTGAAGAAI GGCAGCGGCCACACCAGAAAGTAACCCTCCACCACCACAGCAAACAACCACCACA ЭTGTATAGAGAGGGTCTGTGCTGTGACGTATATACTGAGTGCAATTAAACACTCGA* :AGTTAACAGAGGGCTGTGGAAATGAGGCCTGCACGAATGAGGCTTGTGGCTCTTC 
TACTGTCCATCACTATTTCCATCGGACCTCTATTCGGCTGGAAGGAACCGGCACCG ¿GGCCACTACATACACCCTACAGTACATTGCCAGAATGACTGCCAACGGAACATAG/ TCTATTTTTCCTTTTTACAGCCAGTTGTGTACTACCTGGTGAAGTGGTGCTCTCTAC ¡CAGAGGCAATCATGAGGCCGGCCATCTTGGTGGTTCTGCGTGTGGGGTAGCTCA

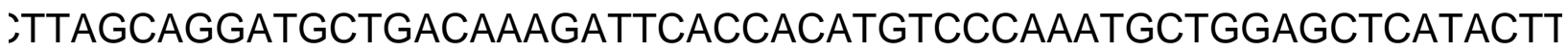
AAAAATGCCATTGTGAAAGTTTGCATGTCGGATATCCAGCTATAAATTACTTCTCAC, CATGTTGTACTCACTGCTGGTGAGGTCTGGTGGGTTGTAGTTGTTGGTAAGGTACA TAGCTGCACAGGGATGAAGGAGATAAGGATGGACAGCGTCCATGCCACGCTTATC. כATTATTTTAAGTCTGCTTGATGACCAAATAAAACTTATTTGACAAAAAAGTAACATA GTACAAAGATGTTACACTGCATGTTGTCAAGCATTTATTTTGCAAAAGTCAAAATCT ITCTCCATAAACAGCCCCCTCTGTGTCAATCTTTCCATCTCGCAGATGTTGAATCCG৷ TCTCCAGGTTCATCGCCGTCTCCATCCCTCTAAATTACAACCGCAAACACGTGGAC GATCATGTGGTGTATGTTCTGTCCAAGTCCCACTAGTAGCATGGTTCTGTTGAAGTI TCCCGCCAGCCACTGAACTGTGATGTTCCAAGCTGCATCCACTGGCATCACGATGF ATCATTATCGGGGCACTTTTCTCCGTCCACCATCAGCCGCCAGCAGACAAGGTCCF ITAAATTTGCAGAGTACAAAGAAGCAGCCAGAATAGCCAAGGAGAAGTCTCTAGAA^ AAATGTACAATCTCATATCTCTTCACAGGAGCCCAAAGAACCCTCAGCAGAAGATCA ;GCTGGGTGGTGTTGGACCGATGCGGGAGACACTTTTCCCTGGTGTTGAACTTCCT GTATTAACAGCATGCCAGAGTTAGTTTCAATCGACAGCTTCGCCTTGCATAACCTAC ITGAATGAATGAATGTGTGATTTACTGAATGAATGAGTCATTAAACGAGTAAATGAG 1 CTTGGTCGGCTCTCCAGAAACCCTGCGCTGCCTGGAGGAGGATTTTCTCCCTTCTC ;GAAGATGAACCGTATTCCTGCGAACCTCCTTGACCAGTTCGAGAAGCAGCTACCG, ITCACAAGAACAAAGAAGATCATATAGGGCTTTCCAGCTGCACGCAGAGTCTGTAAC TGAGCTGCTTCCACACCCACCCAGCACAGGCCCCAGCCGTCCTCGCTACCTATTCC :ATGACCCCTCTTTCTCCATCGAGCACCTTTCAGAGCATTGTGTCTTTGTATGACAAC CACATCTGACCCAACGTTTTGGTTAAAACAACTCATTTTTTGTCATGTGAGGAGGCC ATTTAAAGATGAAAATATCCAGTCGGTGTCATGCAGTTGGATGAATTGAGTCTGGA^ ГGTAGTTGTGAATAAAGTTCATAACACATTACCTATATGTCTGTAGATAGCTGGTGTC CTGTGGAGATCAGGATCTCCTCACCTGTCTGCTGGAGAAGATGAAGACCAGTGCG। TGCCGATGGCCTCAATTAGTACTCCAAAGAGAATTGACGTCCCCGCAGCGAAGTG( ;ATATGAAAGAGTCGATGATGATGAATCAAGAGTACGGGGGAGAGCAGCAGAAAGT GTCCTTCCTTCTCGAGTCTCGTGAGGAGGTTCGTATTTGCTTTGTTTACACGAGAG/ TCTGGATAGGGTACGACATCCAGGATCTCAAAACCTAAGCTACAATTGGATTAAAT\& TAAGCCAGTCCAGCCAGGAAGTCTCGTGGTGAGAGATATCCAGCCACAGGCCTTAC :TCCGGGTTTCCAGCGCATGGATAACAATGCAGCAGCCATCAAGGCCCTGGAGTTA 
GATGATGAATCCATATGCAAGATAACTGAGGAACCCGGTTATGCCATTTTCTCAGC' \AGGAGCATGCAGCAGAGAAGATGGCGTATCCTGGATCTTCGTTAACTCTGCACAC CCTATGAAGATGCTACTTGGGAGCTGAAGGAGGATGTTGATGAAGGCAAAGTGGA AGGGTTTGGTCACACAGAAGTATCGGTCGAAGCTGATGATTAGCAGGTTCATGACT -CTGGAGTAAAGGCTCTGAGTCAGACAAATGCAGAGAGGTTGCGGATGGAGAGGAC AGTTGGTTATTGAGACTAAGAAGTTTAATCTGTAATCTGTGCTCAACAGGTGGTGTG IGGTTATTTGGCTTGGCCACTCTGAGGTAGACCACCTCTGCTGTGTTTTTCAGCGCT ATGATGAACGCCACCTTGGGTGTCATCTTTCGCTCATAGCGAAACGGGCTTGAAAT CTATAAACAGGCATATTGGGATGCAGCAACAGTCTGTGGTTACTTCTAGCAACCCC GTCCACCCTCTCCAGATCTTCCACACTTATGGCAATGTTTAGCAGTGGTGAATGGC' GGTTGGCAGCTGATCTTTGGGGCATCATGGTCAATGTGACGCCCAGTATAGACCC/ CAGCGACAGATTGTCCTGCTCTCCGCCACGTGGATCCTGGCCTTAGCCGTGGCCT CGACTCTGTTGTTGCAATGTGAGTGAGGAAACATGGCTGTGAGGTGGCCAAAATA^ $\checkmark A A G T C A C C A G C A G G T C G G C A A C G G T G A G G T T C A T G A T T A A A A T T C G T A C A T G G G A \prime$ \CGAGCGCAAGTGTGGCGCCGTCCGAGAGCAGTATGGCATCCAGCGGGTGGAGG( $\downarrow$ AGATGGAGCTGGCAAGTTCGCCCTCTCAGGTAAGTCGTGAAATGTCCTGCTACTT। ITCAAACGGGTCATCGGGATCGAAGGGGACTTCATTAAGTAAGTTTGTTGAACAATT GCGGGACGGGACAGTTCCTTTGCCAGATAGCACGAGGGAGCTGGAGGAGGTGTT ¿CAGAGCTTACCAAAATCGAAGCCACAAACAACCCCCGACTTTCCTATATTCACCCA TTGATGAATGAGTGAATGATTAAATGAATGAGTCAATCAATAACAAACTAAATAAATC ¿CGTGTGAGATGTCTGGAAAGGCCTGCGGTTATGAGGGACGCTGCGCTGCTCCTG ATTCATAGGGATGGTTTCCACACGCTGCAGTACCAGCGCACGTCTGCCACCACCAC ¡AGACACAACGCACATACATAAACAAACCAAATTAAAACAGTTTGTATCGTGCTTTAC ¿AAAGACGTTCCAAACTGTGGGGGGAGGAGCCCCGGGCCCAGATGGGGTCATCAC. :ACCACACGTGTGACAGGCTTCAGCCCGCAGATGAGCACAGCTGGTCCAATGAGC( ¿AAATGAAGAAAAAGAAATGAATATAAACGACCGAGCAAAGGTTATCAAGAAAAGAG łTGGATTTAGAGGAGGCTTTCCAGGTGGTTGGAGAGTTCGGAAGTCACCAGAAGCC ¿ACCCCTTGCCACAGTTTCAAGGGCCCCTCTTCTCGCACTATACCTGCAGCTGTGC' GCTCCAGCTGCAGTCAGCGCCCAGGTGAACAGAGTCCCCAGCAGGGCCTGAACC/ ITCCAACAGCGTAAACACATAGATTCCACCCTACAAAGAGAGCAGCAGAGTAAACA GCCGGAGTCTCAAGAGAACGGCAGGCTGGTTGTGGATAGCGTTCCGGAGAAGGA* ATGGAGAAATCCTCTCTGGACGCAGACTGCTGCGCCCTGAATATGGTCCTGGTGG\& †CATTACATTATTTGTGTTCAATATATTGCAAAAAATGGATCAAAAGCTGTTTCAAAA ${ }^{-}$ ¿GGTAAACCCTGACCTCTCTGTAAAGAGTCACAACCCAGACATCCATATTGAAGAG.TATAAGAATAATGCCAAACTGTGTGATCCTCATCCCTCTAAGAAAGGAGCCAGTTC, 
TTTGGGATCTTTTTACCTCCCTCTAGTGGTGATTCTCTCAATGTATTGTCGTGTGTA* CGACTCGTCTTCTGGCATGGGTTCCCTCCATCCAAATAGTGGCCCGACGGAGATAC AGAATTCAGGAAGATTGAAAGCCGCCAACCACGACTCAAGAGAACTGTGAGATACT -GAAGCGTTGCTGACCACGTAATCCAGCGCGAGCCACAGGTCACACACCACTGGGC ¡ATGGATGTGGATAAACCAATCCCAAACTCCATATCCTGACAAACCAGACCATCCAC ;AATCCTTCGGGAAACCACCGCAGGAGGAGCTTCAGACGGCCCGGATACATGCGG -CCCACCGCATCCTCATGCATCACATCCTCCAAACACACATTATTCACCTGCAGGTC GGCCCAGTAGCGGTCCACACTGATGACGCACAGATTCAAGATGGAGGCAGTGGA\& GAAATCTGGCTCCGTGTGACACAATTAATATTTCTTACCTCAAGATACACCACCCAG TCTGGAATGACTCTGAGCATTCATTCACCCCTGGGGGAAACTACAGCCCAGCGTCF $\dashv$ ACGGCGGCCCACGAGGGGTACAACTACCTGGCTTTGATATGTGGCGTGCCTCTC» CCCCCGTGATGTTCGGCATCAACAACGTCCCCAACCGGGACCACAGCGAATGCAA AAGTCGGGCTTAGCGACTGGCGCACAGACGAGCAGTCCGATAAAGGCGATGTGCTI CTTGCGCTTGTTGTTGGTGCTGGCAGCCCACAACACGCCGAGGTTGCACACAGCTI בCATGATGCACACCCTGGACCGCATCAACGCAGACCCGCTCATCCTTCCCAACATC CAGTGTAATGTATGTGCATGAAATGATGAAAATCTGCTCAGATTATTTTAGCAACAC. GTTAAAAATAAACATACAGTTTAAATCTGATGAGGCCAAAAGGGTTTTAATGGCAG/ GAAGGAGGCGCAATACTACAGACTTCAGGGTCTTGTGCAGCACTGCCTCTCCACA AATGCTTTTTCTCAACTCCCAAGACTGGAGTCCTTGATGCTGAATAGCAATGCCCT :AGTAGATCAATAAGTAAATGAATCAGCTCCATAACATGAGAAGAATAAAATAAGTAA GAGTCTGCTGCGACTCGGGTTAGTTTCAGCTCAAATTCAATTGACTCCAGAACAAA' ¿CAACAGCGAGCAGCGCAACGAGAGTCCCGGGCGCATTCGCCACCTGGTGCACTC ¿GCAATATAGATTGTTCCAAGGCAGCTTCCCAGTAACAGGAAGAATCACAGAGTCAr ¿ATGAAAGTCGGCCTGCTGCTATGGGGGCAGAGTTGCTTAGCAAAGACACTCATTC ¿TGGCTCCTACTTTTGTGAGCCCTCAAAACCAGTCCGACTGTCCCAAAGCAGATGA ;ACGGAGAAAATAGAAACAGCAGCAGCAGATCATCATCACTTCACСCСTCССTCTCT ¿AATGATCACAGTGCTGGTGTTCCTCCAGGTAACTAATGATACATGCAAATGCATAA TCAACATGCCCCTGTATTTCTGAGTCTGTACGCTTCCACCATTCTTTCCAGATCTGC łGCGGACTGAGGCCTGGAAACATGCTGTCTGTGGAGAAAATCAGGACACACTGCA1 CGTAGATACCACCCTACAGAGAGAGCAACAGAGAGATGAAGACACAGTGATTAACC TCAGAAATCTGGCTCTGGGCCTGGGCAAGTCTCCAATGGTTATCGCAGTACATCTC †GGAGAAGAAGGGCCATCTCATTCCCAATGGAAACGCGCACCCAAGCGTCAACGG( TGTACCGTTATGTACATTTTAGAAAAAAGCTTTAGGACTACAGCACCAGCACAGTCA ГGCTTATGTTTTTGTTAAAGAATAAATATATAGTTAATCAATCATTAGCAGAAAATAG( AGCCTTCCCGGAGAACAGTGCCAAAGGAGCTCACAACTTCTCTGCTTGCAGCAACC. 
TGTTGTGGCGAGGAGGGAAACGCGAGGACTGATCAGCGGACAGAAAACTGAGAAC 3CAGCTGAAAGGGCCCAGAGAGCTGCCACTGCAGTCATTGCCCTTCGACCCGTAG -GTTTTGTCTGGCTCTTTCTCTCTTCTCACTGGATCATTTTTATCCTGCCTGTATTTTT ¿CAAGCGGCCAGTAACCCTTAATGATGTAGACGGTGTAAAGGTTCATGGAGAACAC

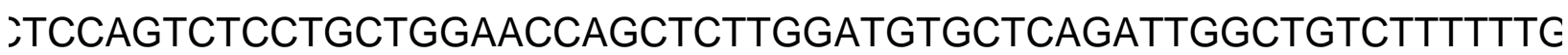
TCTGAGCCGATTAACCAGCTGGACGTTGCAGAAACATCATGTGATTCTGAACATCAr ACAGCACACAACACAAAGAATGACCTTACTGTGTGCTTTTTGAAAAACAAGATTTAT †CACATGATGTCAAAGGCCACCCAAACATCGCAGAAGGCGCCGAACGGCCAAAAAC ;GGCATAACTAATGTGGCCACGAGAAGATCTGCCACCGCCAAGCTGACAATGAGGT łGGCGTTGAAGAAGCGAAGAGGAATTATTATGCCATGCTCTATTCССTGCTCATCСТ ATCCTCATTATCATTTTGGGGAATGTCCTGGTATGCCTCAGTGTGCTGACCGAACGC GCTGGAGGACAACAACTACGTTATCTACTCCTCCGTCTGTTCGTTTTTCGTGCCGT، GTACCCCAGCAGAGCGGGACTGTAAAAGGGCAGAGTGCTGAAGTCGTGGCCAAGC GACACTGCGCAAAGAGTGAAGGTAATTATCACCCTCACTTTTGCTGCCATGGAAAA* :ACCCTAGGCTGTGAAATCCGAGACTCCTGCTGGCATTCTGCCGTGGCTCTGGAGC ACTAGCAACTGTTAAGTAAACACCCTGATTACCTTTGCAAAT

łGCGTGTTTTCTTGCCACTAAAATGTTGCCTGAACAGTGTTTACACAGACTAACAGG ГTACAGGTCAGGCGTGTACCCTTAGACTTACTATGTTT

TAGGGCCCTTCATCACATAACAGTGGAATCCTTGCCTAACCTTCAAGAGGTGAGCA' AGAGCAGCAAAGTAAAAACACACAGTGGTTAAACATGTCTAGCTGTGTAACATATC। CCACTGTTATACAATGACTTGCCTAATTACCCTAACTTTACCCTAATTACCCTAGTG/ :TGTCCAGAAGCTCTTCACCAAATCGCACTCTCTTGAAGGCTCTTCCAAGATGAACG GTGATGAAGACTTGCCTA

ACTGGGTGAGGAGCCACCAATGGGAGCACCACTTGACCCTGGCAGGAGATCGCC` CCAGCTTCTGAACGAGAATGTGAAAGTGGGTCTGTTGTTTGCCTCTAAAGCAACAG -ACCGACAGCAGCTCCACCGTAAACCGCCTCGGTTTTTTATTATTCTCCGCCGGTCC. ACAGAGGACTGACATGTGTTTATGTAGGTAAATGTATTTGCATTTCCGATAGCACG, :CTTCACCCTGGATCTGGAGTCTGGTTTTGGTAAGATCCAGGGGGAATGTGACTAA^ ГTCAATACACTCACAGAGAGAGACGTGTCTATATCTTTGTGTGTGTGTGTGTGTGTG ¿CTACGATGAACATTTCCCCAAGT

:TCAAAGCCCCAAAGAAGGTGCGGGCACTGGGACGGACAGGGTCAATACCCCTG ЭACCCTCAACGGGGGGCCCATGTCAGCGGCTACCGGAGCCATCTCGGCGGTGGA। GAGTAA

ЭATGAAACTCTACTTTTGTAAGTGTCTTCACTGGTGAGCGTAAAAAAAAAATGGACA ;GAAAGATGAACCATAAGGACCTGCCACCCACAAGGGAGGACTTTAGAGGTGAGTA 
iTCGGATCACGCTGAGACCGTGACCTTGCGAATACACCGCGGAAACATGACGGTG1 CAAAGGAAGGGTATTGCAGCGGGTAACTGACGGCCATGCAGCGATCCACAGAGAT TGGTTTTCATTAAGAAACAGACGAAACCGTATGAATTATTAACAAGTCTTGCTCTTC ACCGATGATCAGATCTGCACAGGCCAGGCTGAAAAGGAAGTAGTTGTTGACCGTC' ;GTTTCTTTTCAGGCTCTGGACGTCTCATGAAGAGCCAGCGGGGAATGTGGTCCAG IAGAAGTCCTATTTCCAAGTCGCCTGCTGTGGAGAACACTCAAAAGAGTGCTTCAG. TCGGTATAATAACCACACACTATAAATCATTTATTAAACATGAGCAAATAGTGAATTC ¿CTACAATCTCCGTGGCGGCTTTCCATGGCATCACCAAAATGGCCACAAGCAAGTC, AATTAGTGGTGGTCTGCAGCGCTTTCTCTCGGGAGACTGCCATACACACCAACAC/ -GGCTATCGTGTTTGGGAATGTTCTGGTTTGCATTGCTGTGCTTCGAGAGAGAGCTC ¿TCACTCAAAACAGCAACCAACTACTTCATTGTCAGTCTGGCCGTGGCTGATCTCCT उCCCCATCATGCTGCTCCTGTACTGCGGAATGTTCCGGGGCCTGAGGAACTGGGA ¡TCTGTGTAGGGCGATGGGATGCAGATGGGGTGATTCTCCACTGGCAGAGATGGA' TGTGGGCAGCTGCAATGCCGCTTCACCTGTGTTATTGTTACACGTGGGCAAATCAC :AGAGCATTGAGTTCATCCGGGACACTCTGGTCTCAGCCGAGGAGGAGGAAGGCAT

CCTTGATATCACCATAAAAATAAAGCTTGAAA

TCCACTCCAACCCCATTTACTGTGACTGTGTCATTCGCTGGATCAATATGAACAATA CTGCTTTCTGGAACCCTCTGATGTGTTAATCTGAACTCACTATATCAGCTCAACAGT AAGCCTTTACATGTCACTTTAAGCTGAACACTAGTGTCTTGAAGAATATCTAGTCTA\& GCACCAAGGGAGACTCTCGCTCAGACAGCGGACACCACCACCATCACCACCATCA

TGTGGGAGGTGCCAGGTAGGACAGAGCTGCTCAAAACTGTGCCAACTTTAGCAAG( TACAGCTAATCACTAACCCCTTCATAGGACCACTAACCAACAGGTACAGCCGCTAG ¿ACCGTCAGTCTCAAAGCTCCGAGCCTCGCCATATTCTCTCGCCTGCTCACAGATT/ AGCCGGCAATCTATGCAAAATGGATATATTGAGGGTCAAACGTTGTCTT ICAAAAACATTATTAATAAAACAGAAAGTAGACAAAAAAAATATTCAAATACATTAGG iTGTGTGTGTGTGTGTGTGTGTGTGTGTGTGTGTGTGTGTGTGTGTGTGTGTGTGTc

כAACATTTAGGACTCTGGTGGTCCAACAGACGAGCCTTGATCCACCTAGGGAGACC GAAGAAGAGGGAGGGCTACCCCGAGCGAGAGACGTGGACCAGACAGATGGACTT(

AGCTCAAAATTTTTTAGTTTGACTTGGCTTTACACCTGT ACGGTGTTATGTTTGTAGACATTTTATCAGAACTAGTTTGATTAAAATTCCTTAAAG ${ }^{-}$ 
ГCCGAGGACGAGGCGTTACGCAACCGGACACACTTCGCACTGCGTCTGCTCAAGT TACGCAGAGGCTGAGGATGGATGCCGTGCAGCACAACACGTCAAGAGCGGTCCAr :CATCAGCCTCGGCCTG

TGCAGGTGTCGATTAACCTTGATTGAGAGCATGACGAGGATGTTGCCCACGACGG GAACACCGAATGAACCCAACATGGCATTGAATGCGTGCTAGGTGAACGATGGTGC, AATTACTTGGCGAGAAATGGCTGACAGAGGGTTTCGAGAGAGACAACTCATCAAAG ¿ATTATTTATTAAGCATTAACTCTACATTAGTAGGTGTTAGTAAGCAGTTTATAACTA AGAAATAGCCAGTGATATAACAAAGAAGTTGGTGACTTTAGAGCGCAGATGGCGAA ITTGCCAAAGACAATGACAAAGATGAGGAGAGTCAGGAGCATGGCATAGTAGTTAT. ¿TTCAGACCACCACCAACTACCTGGTGGTCAGTCTGGCAGTGGCTGATCTGTTGGT -GCTGGCCATTCTCGTTCTGCCGCTTTATGTCTACTCTGAGGTGAGGACCAGAGAT1 AGAGGCTCGGAAAGCTAAATTAAGGAGCAACATGGAGGCCTGCCGAAAGCTTCAG GAGGATGAGTTGAAGATGGGGGAGAGGATGTGACCTCCCACACGGCCTGAGTCC/ :AACTGCCATTCAGAGTATCTGCAGTCAGCTGGTGAAACATGATGTTCTCAGATGTTI ГGGCTAAATGTTCCACCGAAGGCGGAGGGACTCCCATGAAAGGGAAGAAGCCTATๆ

،CAGGGTCCGCTTCATGGAACTGGATGCCCTTTTATGCGCAGGGCCTTCAGAGTT AAACCTGAGTTCTCTTCTCTGCAGAGGGGCTTCAGGTTCCGCTATGGGTGTGAGG \TATTATGTGCTGTCATCATGGAGAAGAGGAAACACATCAGCTATTAGAGATGAGTT TCACGGCCATGACCATGGGAGCCACAAACACAGCAAACGAAGTAAGAGCAAGGAA

¿ACACGCACACCTGCATGAAACACTGCACAAATTTCAATATCATTTAGCAATACACC TTTAA

\AACTCCATCATGGAGATGCTGGGGAAGAAAGAGCGGAGAAGCAGAGATGTGAAG।

CAGATATCAATGAATACAAATGTATAGATATTATTTGCTTAAAGATACATTTATTTAA כAGAGAGCAGATCCTGTTTATCCAGGGTGTCTGCAAATACAGGACAATGAAACACC.

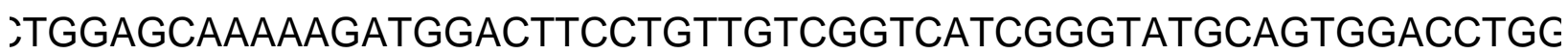
¿ATCATGTCCTGCGTGGGTTTTGCAGTGGGGCTGGGGAACGTCTGGCGCTTCCCG 
ГCTCAAGGGAAAAGAAGGCCGCCAAGACTTTGGGTATCGTGGTGGGATGCTTCGT/ GGCGTTACATAAATGACGACCGAACACCCAGCGACCCAAGGCTTCAGATACAGCAC

IGACGAAACTGAGAGATCCAGTGACCAGTGCGATGAAGACCATCTCCACCGTCTTG ACGTTGAGCACAAAAACGGTAATGACAATGGA ;TCTTCAAATAAACACCATTTGCATGATGAGGAGGACAATTTACCAGTGCAATCACG' AATCTACAAATGCTGTATTCTTGACTTATAAGCTCATGTGGTTTTATACTTTGTTAAT( ACTTTGTGACTGCAGCACAGACCAACGTGTTTCCCAGTAAGGTGGTCAGAATGAG( ACTGATGCTTGGCCTCACAGTCTGTGCAGTTGAGAGCCCCGGTCCCATTGTCATA^ CGCCTCACTGGTCATGCCTTGGGCAGTTTATTTGGAAGTAAGTACACTAGGAACTA ГGATGATGGAAGTAGGTGGTTTACAATCTCTGTTTGACTCTAAGCATGTGATGGTAT GAGGCCGCAGCGTCGCTCCAGCCGCTGTCTGGCCTGCCGCCGCCGCTCCCGCCT \CTTCCTGCAGCTGAAGAAGAGGACTGTCTCCTTCGGGATACTCGGACATGGTGAA GGAGAGCTGTCATTCATCACTGAAGAGCGAAAGCGTGTGTTTGCTGCATTTGCTCG ГGTGGGTCTGATCGGGCCTGGATCCAGCTCTGTGGCCATCC

TGAAGGTCGTCTTGTCAAGCAGGTGCATTCACGAGAAATGG उCCCGTCTCACGGAGGGCTTCCAGGGGCCTCCAGCGAGAAGAACCGCAAGTCCTf ATTAAAATTAAAACAGAAATTGGGGAAAAACATAAAAGAATTAAGATTTAATTACTG/ .CGCAAGTCAGACTCCAAGCAACGCTCAGGGATAGCAGGCTGGTGGAGCTCTGACC

CAATTGACTTTTGACACCCTTAGCCACACATCTGGTTAAACTGCCTTTCCATA

GGTTTGCTGAAGAGGAACTGGATTCTCATTGCAACCATCATTGCGGTCATTCTGGG

ГTATACATATACATATTTTTTAACTACAAAGGTTAAGTAGCTATATGTTTCTGTTAAAT ACAG

;GCAATGTGTGGCGCTTTCCCTACATCTGCTACCAAAATGGAGGGGGTAAGGTCAA TACCTGTGCTACAAAAACGGTGGAGGTAAGAACTGCCGGTGTCTCTCTGCAGCGC 
łCTCTGTTGGCTTCCGTTTTTCCTGGTTCTTCCGATCGGTGAGTTGTTAGAACTTTT ¡AGAATGGAAGAACCACAGAGCTCAGAAGCAGGTCTGCGATTGCCAGATTTCCAAT

iTACGGGCTCTCGCCGTCGCCCTCAACCGCTGCACATGAGCCATTGGCGCTGCCAC

TGATGTTTTCAATTCCAGTTTCAGTTTCATTCAACAGTCGCTAGACACTAGCGATTT/ ЭATTCATTTTTCATTACTCAATTAAGTGTTAACGATTCATGACCAACTAAACTTCTCT( כAGTGAGAGGAAACAACCAGTCAAAACTCGCTTTGATGAATCGCGCTGCGACACAC ITAAGTGTCATTGTAGGGATACTCCGTGAGGAAATCCATGAGCTGTGGCCAGGGAA ¿CTGTAGTTTACCATGTTTAAGGAAAAAAACAATAAATTTTCCCATTCGAAAATCTGA` ACTTTTTCCAATTGCTGAATCTTATTTGATGATATAGTCACTGCACTTTTATAAATTA' GTTATAGAGAAAGACATTACATTGGAGGAGCTGGACCAAGATCACTATCCCGATCC .GGCGGATGAGTTCAGAGCTGTCGCATCAGTAACGGGGGACTGTTGATGTGTGTCA ;ACACCCTGCTGTACAGTGAGG

\CCCACAAGTGCAGGTAAAGTTTTTAATTATATATTTGTATTTAACATTTATAAGATTT ҰAATAACATGTAATATTCATGTTTAGGTATTGTCTCTGCAATAGTTTCCAGTGGAGCT ¿ACAACCTGGACAGTGACAGCACCTACCGGACTCCCAGCATCATGAGTCGGCACC/

TAAGAGACACCGTTCATTTATTGAGCATGTTTCTCAATGCATATTTGTTGCTATCAT1 GAAATGAATGAATGTAAATGCATCTACCCAATTCTGCCACAGCTGCAGCACAGGCT AT GTTCATGTTCAGATCTTTCGGATGACACGCCGTCTGTCACTCCCCСTCCСССTCTC। 
ГATATTTCATTATAGTCAATACCTGTCAATGTTGGGA

AAAATAATGAGTCACGGATCGAAGG

¿TGTCACAGCTGAAGTTGGCATCACTGGACACGTTGCTCAAAGAGCCTGAAACACA

ATTAGATGTAAACACATGCTATTCACCCAGAACTGAACACAAACAATCTGAATCAGC כACCACATTTCTAGAACTGCTGGATCTTGCA

:TGCTGTCCAGGACTGTGGAGTAATTCACATCCATGGCTTTTCACCGAGTCGAGTTA GAAGCCTGAAACTGAGAGACATAATTCACTGTCAGTCAATGCAACAAACAAAGGATI ГGCATGGAAATATAAAAATGACAAACAAGTTTATATTTGAATTTGATCTATGTAAAATI GAAAAAGAGGTTGAAAAGATGGATTCATGGCAGC :CGACAGTCCTGATCCTGTGTCCATGTTGG ITGGTGAACTTC

'GTCGACGTTTATGTTTTCACTATTTCTCTTCATTTTTTTTTGTTTTATTACAATTTTAT' -GATTGTGTGTGTGTGTGTGTGTGTGTCTCAGAGGGCTGCAGTGTGGACCAGTCGT tCGGAGATCATATCACTCACT

ГGTTGAGCTCTAATACGTCTCGTCGTTAATTATGTATCGTTTTATTATTGCTTCACTG` GATAAAGTGAACTT

GAGTGACGTCACAGACACGGTGGAGAAC 
AGCACACACCGTGATATAAATTACACAGCAGCAGATATGTCTGGATGCAGTCATTG, GTCAGGGCACCAGCTGAAGTCTAAAACTTCAAATTCAGGCTTTCTTAAACCTCCATC IGCAGTCACCCCATAATTCCAGACCAAACTAAGAGTGCTTACACATGAATCGGAGCF GAAGGAAGCAGTAACACGCCAAGGCAAAAAGGAACGATATTGATCAGAAGCTTCCC GTTATAAATGCAACATATTTCAAAATATGGCTAAATGTACGCTTAATTTAATTTCAGA'

TTAATATTTTTCACTAACATGTTAATTTGTGTGTATAATTATTGACCTGTGATCTTCA\& -GTGTGGACGGAGATGCTGACGCTGCAGCTGTCAATCAACCGGCCAACAGCCCAG/ 
AAAATAATCACAAGCTGATACGAATATAGCTGATACAAATATAGTGAGTGTATAGAA

¿TGATTTAATGAATCACTTGAGCCAATCAGAAACCAGCATTGTTCAAATGAACCAAT1

\ACTGGTTGATCTGGTTTGTCCGTAGTTATGCTG

¿CATCATATCTTGAGTTGTCATT

CCATTTGACCTATGTTGCAA

†GCTGCTGACTAATCTAATGTACATGCTAGGGATGCACTGATATGG

ATCTGCTGCTGAAGCTCCTGCACCTGTCAAGCCACGCACACCCCTCTAGAATCCAC 
GGAAACAGCATGACATTCATTATGA

ГAGAAACCAGAACTGTCCCGGTAAGCCAATCAAAATCAAGC

CAATGAAACGCCAGAGCTGTGACATCATCTCAGGAGGAGCCGCCCACCAACCACA

¿GTGTATGTGATGAATGAAGGCATTGATCTGTATGTGTGTGTGTCCGTCTGTATATG 
bioRxiv preprint doi: https://doi.org/10.1101/350314; this version posted June 19, 2018. The copyright holder for this preprint (which was not certified by peer review) is the author/funder. All rights reserved. No reuse allowed without permission.

CGACTCGCCACCCACTTTTGTAAACCAACTTTGTAAAGGCGCTAGATA

;TGTGTGTGTGTCCAGATATTTTACTACTGCCACAGAGAAACGTGACCAGACTCCCA 
bioRxiv preprint doi: https://doi.org/10.1101/350314; this version posted June 19, 2018. The copyright holder for this preprint (which was not certified by peer review) is the author/funder. All rights reserved. No reuse allowed without permission.

IGTATGACACTGTAAAATCCC 
Supplemental File 1

\section{adra1aa}

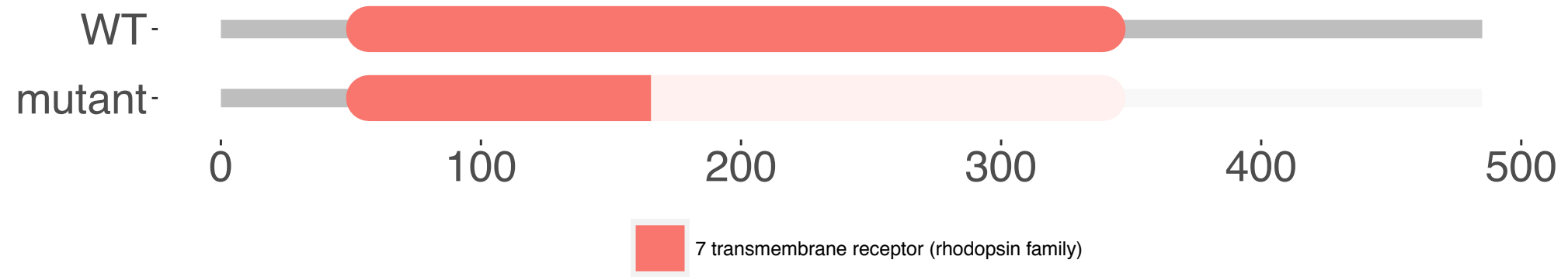

NM_001324454.1

NV1180

NM_001324454.1

NV1180

NM_001324454.1

NV1180

NM_001324454.1

NV11 180

NM_001324454.1 NV1180

NM_001324454.1 NV1180 gagcgacgtgcattgcttgcgctcatagctttatgggtactgtccatcactatttccatc gagcgacgtgcattgcttgcgctcatagctttatgggtactgtccatcactatttccatc

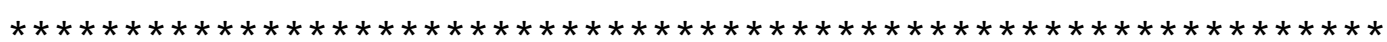
ggacctctattcggctggaaggaaccggcaccggatgatgaatccatatgcaagataact

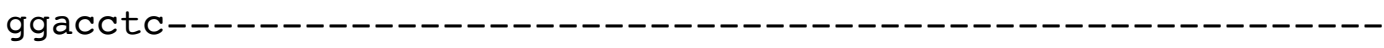
$* * * * * * *$

gaggaacccggttatgccattttctcagctttgggatctttttacctccctctagtggtg

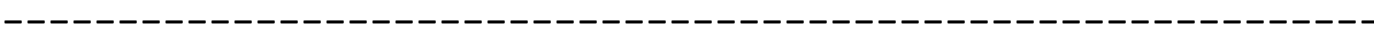

atcctctcaatgtattgtcgtgtgtatgttgtggcgaggagggaaacgcgaggactgatc

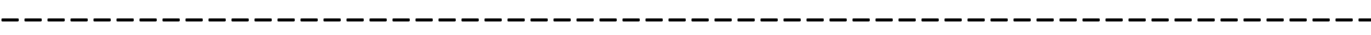

agcggacagaagactgagaagtcggatcacgctgagaccgtgaccttgcgaatacaccgc agcggacagaagactgagaagtcggatcacgctgagaccgtgaccttgcgaatacaccgc

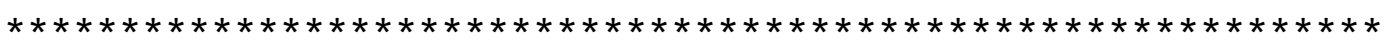

VLCCTASIMSLCVISVDRYIGVSYPLQYPSIVTERRALLALIALWVLSITISIGPLFGWK VLCCTASIMSLCVISVDRYIGVSYPLQYPSIVTERRALLALIALWVLSITISIGPQRTED

$\star * * * * * * * * * * * * * * * * * * * * * * * * * * * * * * * * * * * * * * * * * * * * * * * * * * * * * *$
1560

1560

1620

1567

1680

1567

1740

1567

1800

1627

180

180 


\section{adra1ab}

\begin{tabular}{|c|c|c|}
\hline 0 & 300 & 400 \\
\hline \multicolumn{3}{|c|}{7 transmembrane receptor (rhodopsin family) } \\
\hline XM_680297.7 & ctgctgtatctgaagccttgggtcgctgggtgttcggtcgtcatttatgtaacgcctgga & 600 \\
\hline NV1175 & 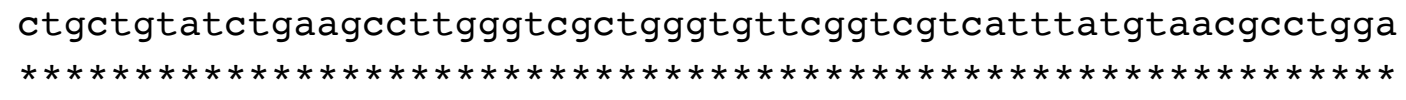 & 600 \\
\hline XM_680297.7 & ccgctcttgacgtgttgtgctgcacggcgtccatcctcagcctctgtgtgatctctgtgg & 660 \\
\hline NV1175 & $\begin{array}{r}\text { ATTTATGTAACcCatcctca- } \\
* * * * * \quad * \quad * * * * * * * * * * * *\end{array}$ & 638 \\
\hline XM_680297.7 & atcgctgcatggccgtcagttacccgctccaatacccttcctttgctacgggtcgaaggg & 720 \\
\hline NV1175 & $\begin{array}{l}\text { atcgctgcatggccg- } \\
\star * \star * \star * \star * \star * * * * * *\end{array}$ & 653 \\
\hline XM_680297.7 & caatgactgcggtggcagctctctgggccctttcagctgctatctctgtcgggccactat & 780 \\
\hline NV1175 & 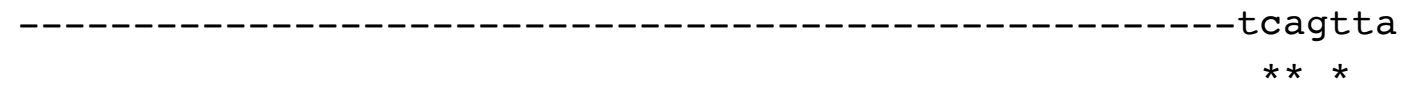 & 660 \\
\hline XM_680297.7 & ttggatggagggaacccatgccagaagacgagtcggtgtgcagagttaacgaagatccag & 840 \\
\hline NV1175 & 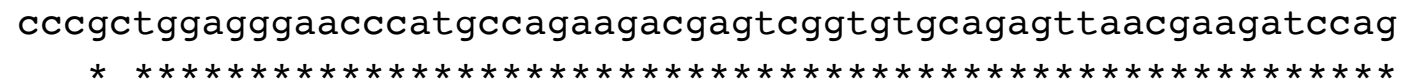 & 720 \\
\hline
\end{tabular}




\section{chd8}

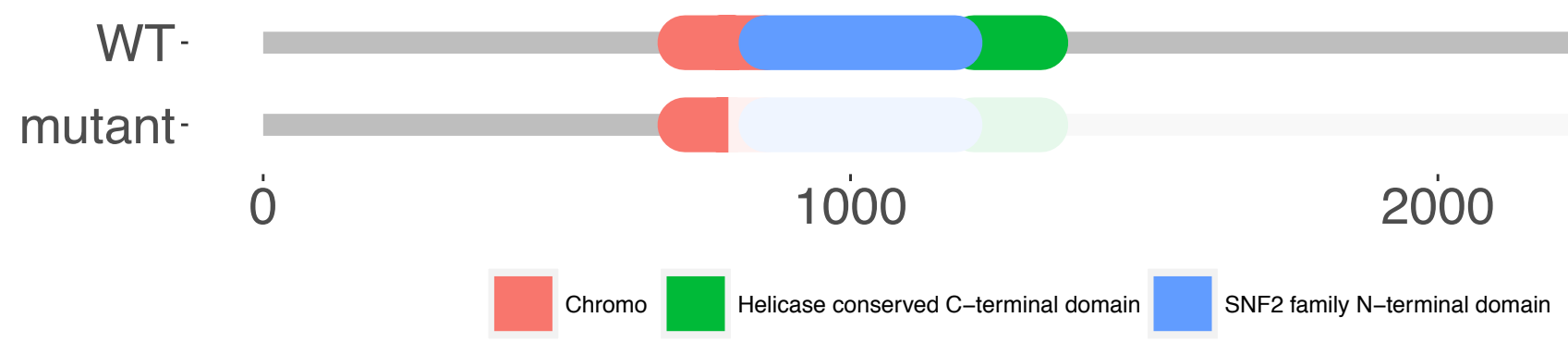

XM_005171312.3

tgttgatgaaggcaaagtggaagaattcaggaagattgaaagccgccaaccacgactcaa 


\section{chrm4a}

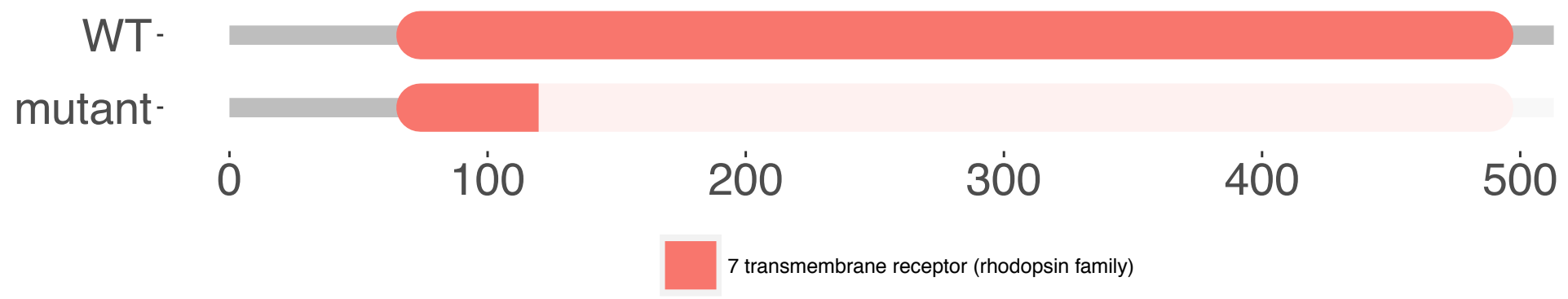

XM_001922407.4

nv1152

XM_001922407.4

nv1152 accgtctacatcattaagggttactggccgcttggcccagtggtgtgtgacctgtggctc accgtctacatcattaagggttactggccgcttggcccagtggtgtg--------tggctc

960

953

GYWPLGPVVCDL-WLALDYVVSNASVMNLLI ISFDRYFCVTKPLSYPTRRTTKMAGLMIA

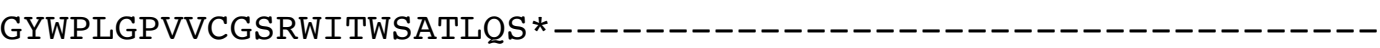

$* * * * * * * * * . \quad *:: . .:$. 


\section{chrna2a}

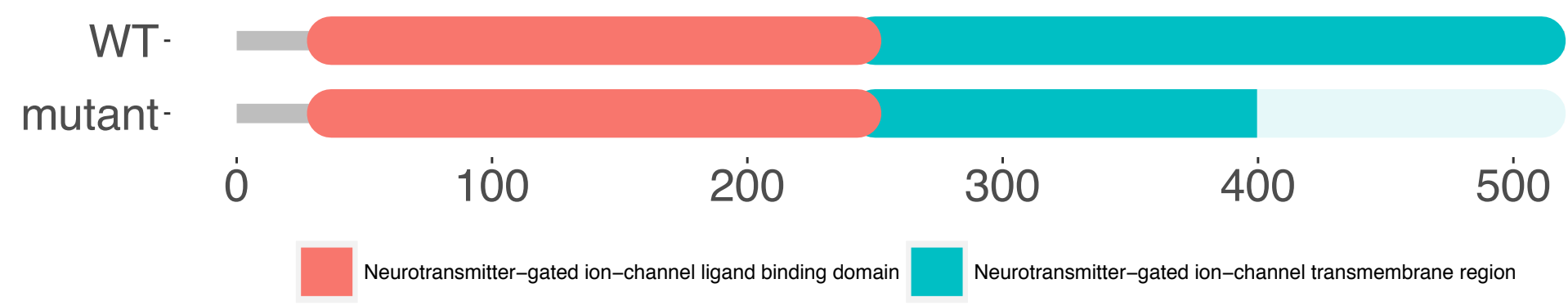

NM_001040327.1 NV1187

NM_001040327.1 NV1187 gggattggtttatccacatccatctcctctccatccgcaacctctctgcatttgtctgac gggattggtttatccacatccatctcctctccatccgcaacc---------tttgtctgac $\star * * * * * * * * * * * * * * * * * * * * * * * * * * * * * * * * * * * * * * * * *$ $\star \star * * * * * * * *$

PEPEKKPKKTANLSTSKSWFQQETGVDGLVCQDMEFGIGLSTSISSPSATSLHLSDSEPL PEPEKKPKKTANLSTSKSWFQQETGVDGLVCQDMEFGIGLSTSISSPSATFV*-_-_-_-

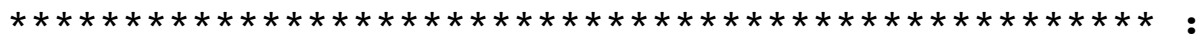




\section{disc1}

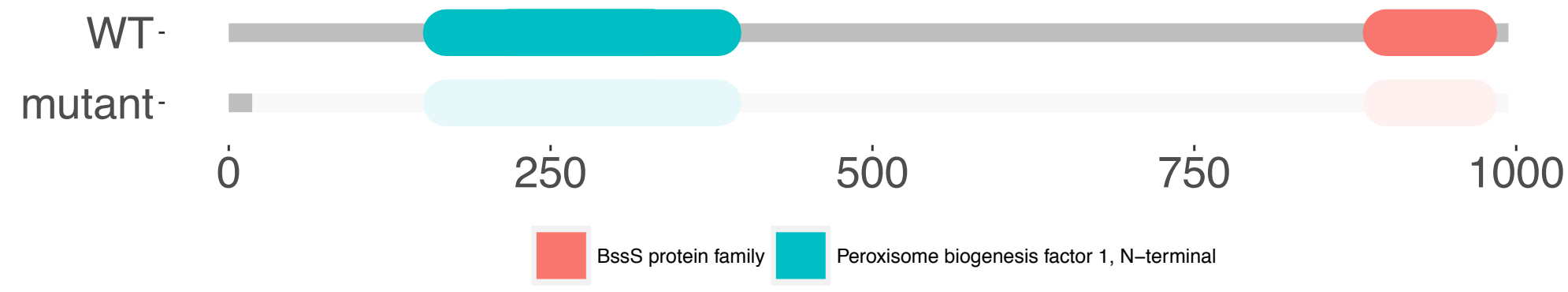

NM_001142263.1

tgtgaatccttcgggaaaccaccgcaggaggagcttcagacggcccggatacatgcggtc 


\section{dlg4a}

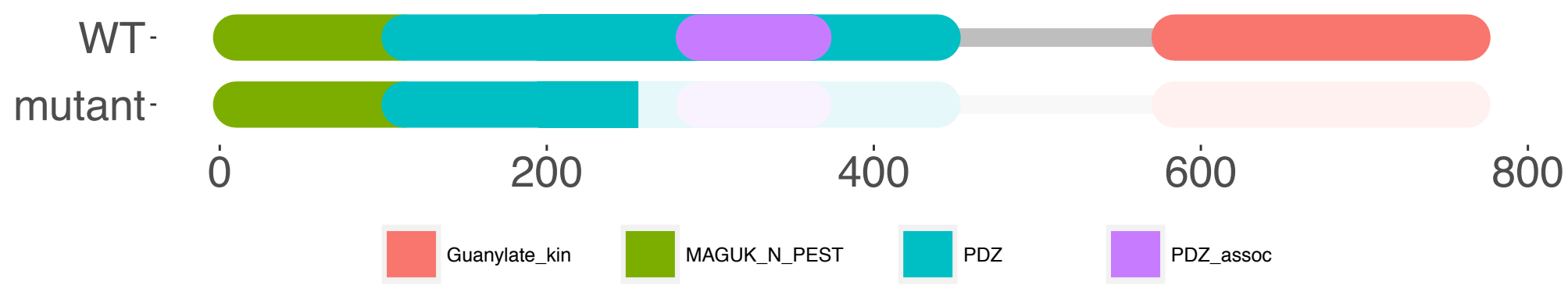

XM_009302021.2

NV1149

XM_009302021.2

NV1149 cggtgaataatgtgtgtttggaggatgtgatgcatgaggatgcggtgggagcgctgaaaa

cggtgaataatgtgtgtttggaggatgtgatg-----------cggtgggagcgctgaaaa

KIIEGGAAHKDGRLQIGDKILAVNNVCLEDVMHEDAVGALKNTAEVVYLRVAKPNNLYLT KIIEGGAAHKDGRLQIGDKILAVNNVCLEDVMRWER*_-_-_-_-_-_-_-_-_-_-_-_-_-

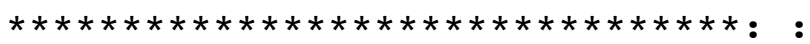

1620

1610

300

276 


\section{drd1b}

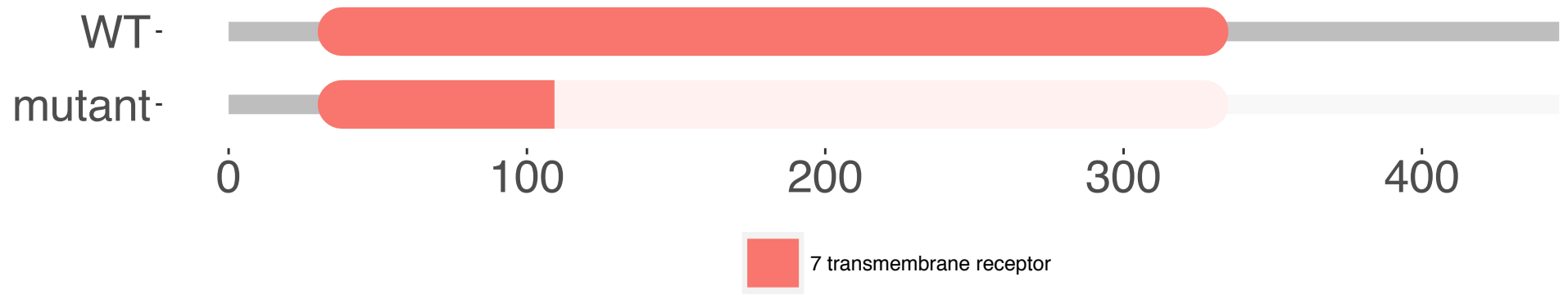

NM_001135976.2

NV1145

NM_001135976.2

NV1145

NM_001135976.2

NV1145 tcagtgtggaccgctactgggccatttcaagcccgtttcgctatgagcgaaagatgacac tcagtgtggaccgc----gggccatttcaagcccgtttcgctatgagcgaaagatgacac

VISLAISDLLVAILVMPWKAATEIVGFWPFGAFCDVWVAFDIMCSTASILNLCVISVDRY VISLAISDLLVAILVMPWKAATEIVGFWPFGAFCDVWVAFDIMCSTASILNLCVISVDRG $* * * * * * * * * * * * * * * * * * * * * * * * * * * * * * * * * * * * * * * * * * * * * * * * * * * * * * * * * * *$ WAISSPFRYERKMTPKVAF IMISVAWTLSILISF IPVQLNWHKAQTTSYTELNGTYGELP -----PFQARFAMSER * 


\section{$\mathrm{drd} 2 \mathrm{~b}$}

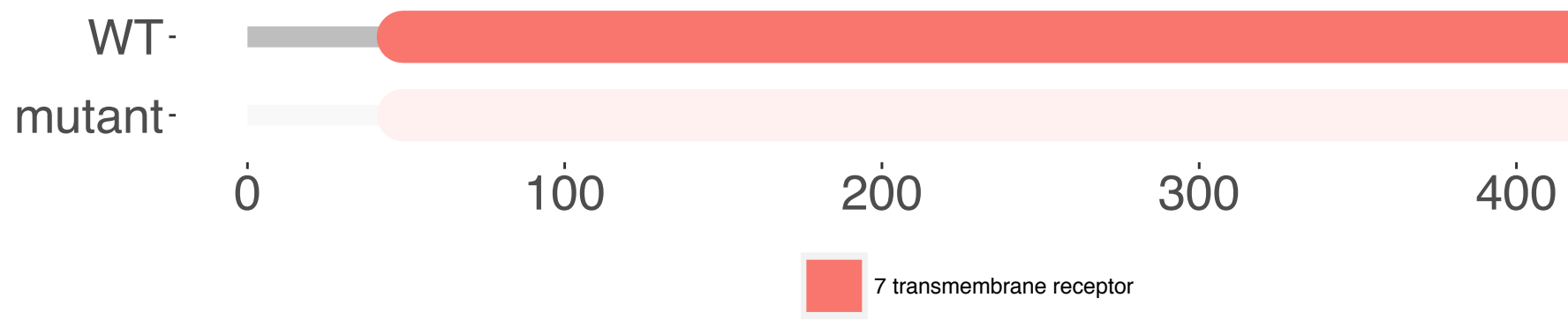

XM_009301838.2

NV1147

XM_009301838.2

NV1147

XM_009301838.2

NV1147 tgacaatgggaccggggctctcaactgcacagactgtgaggccaagcat----------tgacaatgggaccggggctctcaactgcacagactgtgaggccaagcaTAGTTAGCAAGt cagtataactactatgccatgctcctgactctcctcatctttgtcattgtctttggcaat cagtataactactatgccatgctcctgactctcctcatctttgtcattgtctttggcaat

MDFLTEYPYNDTYYDNGTGALNCTDCEAKHQYNYYAMLLTLLIFVIVFGNVLVCMAVSRE MDSFTLLEEKMLSIKKLWI *

** 


\section{drd3}

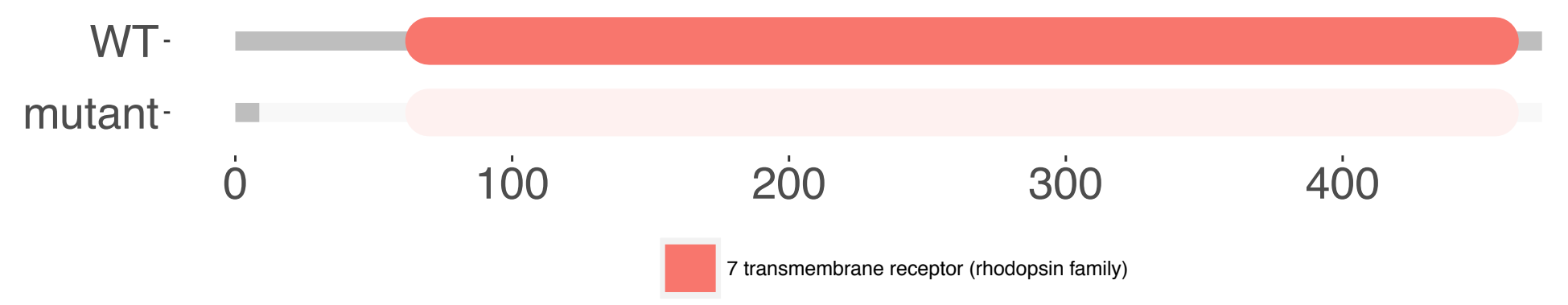

drd3-001 AAGTGCGTTCAGATGGGGTTCTGTTTCGGTTCTTCGCTGGAGAAGCTTAACAGATCTTCC 300 NV1227 AAGTGCGTTCAGATGGGGTTCTGTTTCGGTTCTTCGCTGGAGAAGCTTAACAGATCTTCC 300 $* * * * * * * * * * * * * * * * * * * * * * * * * * * * * * * * * * * * * * * * * * * * * * * * * * * * * * * * * * * *$

drd3-001 ACACTTATGGCAATGTTTAGCAGTGGTGAATGGCTCTGGAATGACTCTGAGCATTCATTC 360 NV1227 ACAC------------------------------------------------- 304 $* \star * *$

drd3-001 ACCCCTGGGGGAAACTACAGCCCAGCGTCAGGCGTTGAAGAAGCGAAGAGGAATTATTAT 420 NV1227 ----TGGGGGAAACTACAGCCCAGCGTCAGGCGTTGAAGAAGCGAAGAGGAATTATTAT 359

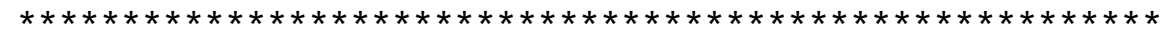

drd3-001 GCCATGCTCTATTCCCTGCTCATCCTGGCTATCGTGTTTGGGAATGTTCTGGTTTGCATT 480 NV1227 GCCATGCTCTATTCCCTGCTCATCCTGGCTATCGTGTTTGGGAATGTTCTGGTTTGCATT 419

drd3-001 GCTGTGCTTCGAGAGAGAGCTCTTCAGACCACCACCAACTACCTGGTGGTCAGTCTGGCA 540 $\begin{array}{rlr}\text { NV1227 GCTGTGCTTCGAGAGAGAGCTCTTCAGACCACCACCAACTCAG--0--0-TCTGGCA } & 469 \\ * * * * * * * * * * * * * * * * * * * * * * * * * * * * * * * * * * * * & \end{array}$

drd3-001 GTGGCTGATCTGTTGGTCGCCTCACTGGTCATGCCTTGGGCAGTTTATTTGGAAGTGGTC 600 NV1227 GTGGCTGATCTGTTGGTCGCC-----TCACATGCCTTGGGCAGTTTATTTGGAAGTGGTC 524 $* * * * * * * * * * * * * * * * * * * * * \quad * * * * * * * * * * * * * * * * * * * * * * * * * * * * * * *$

ENSDART00000130568 NV1227
MGFCFGSSLEKLNRSSTLMAMFSSGEWLWNDSEHSFTPGGNYSPASGVEEAKRNYYAMLY MGFCFGSSLEKLNRS STLGETTAQRQALKKRR-------GI IMPCS I PCSSWLSCLGMF- 


\section{drd4-rs}

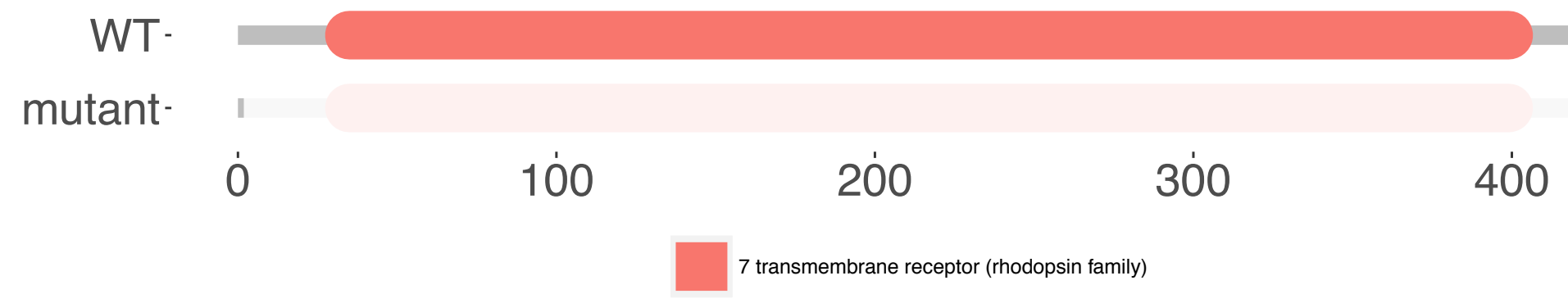

NM_001012620.2

NV1217

NM_001012620.2

NV1217

NM_001012620.2

NV1217

NM_001012620.2

NV1217 agtatagacccaacggcggcccacgaggggtacaactacctggctttgatatgtggcgtg

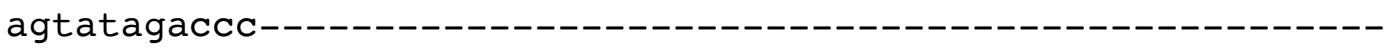

$\star * * * * * * * * * *$

cctctcatcctcattatcattttggggaatgtcctggtatgcctcagtgtgctgaccgaa

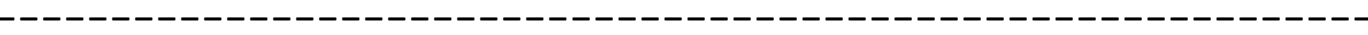

cgctcactcaaaacagcaaccaactacttcattgtcagtctggccgtggctgatctcctg

----------aaaacagcaaccaactacttcattgtcagtctggccgtggctgatctcctg

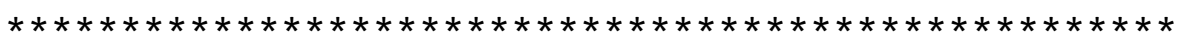

MVNVTPSIDPTAAHEGYNYLALICGVPLILIIILGNVLVCLSVLTERSLKTATNYFIVSL

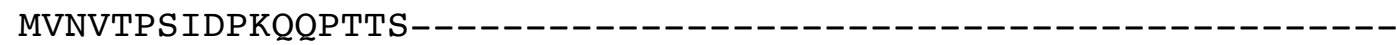

$\star * * * * * * * * *$. 


\section{drd4a}

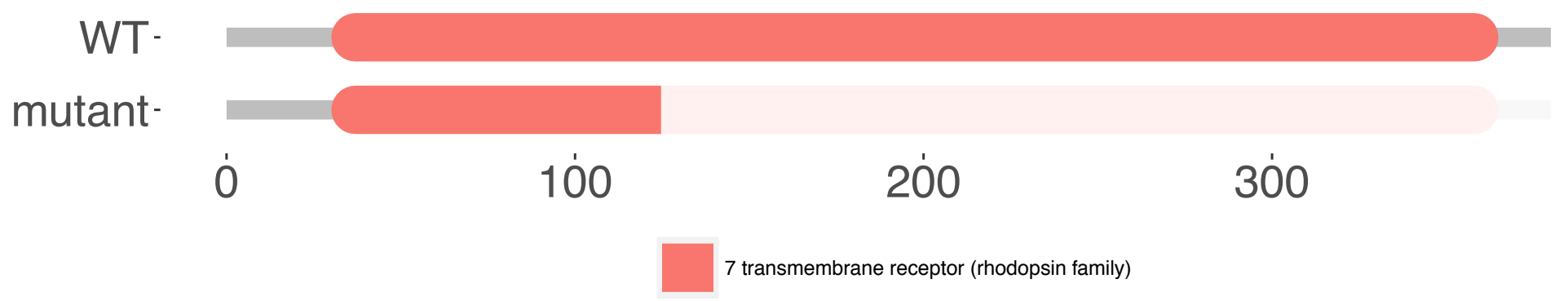

NM_001012616.3

NV1218

NM_001012616.3

NV1218

NM_001012616.3

NV1218

NM_001012616.3

NV1218

NM_001012616.3

NV1218

NM_001012616.3

NV1218 acaaccgcaaacacgtggaccagcgacagattgtcctgctctccgccacgtggatcctgg

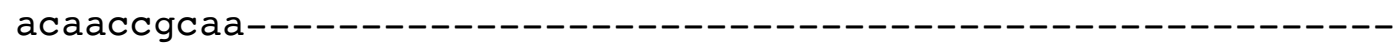

$* * * * * * * * * *$

ccttagccgtggcctcccccgtgatgttcggcatcaacaacgtccccaaccgggaccaca

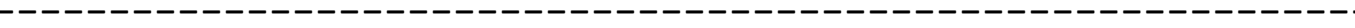

gcgaatgcaagctggaggacaacaactacgttatctactcctccgtctgttcgtttttcg

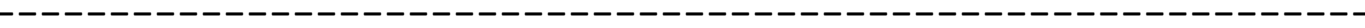

tgccgtgccccatcatgctgctcctgtactgcggaatgttccggggcctgaggaactggg

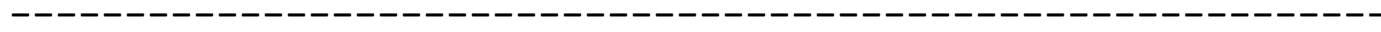

aagaggctcggaaagctaaattaaggagcaacatggaggcctgccgaaagcttcaggagg

--gaggctcggaaagctaaattaaggagcaacatggaggcctgccgaaagcttcaggagg

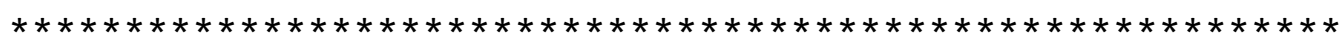

IAVSIPLNYNRKHVDQRQIVLLSATWILALAVASPVMFGINNVPNRDHSECKLEDNNYVI

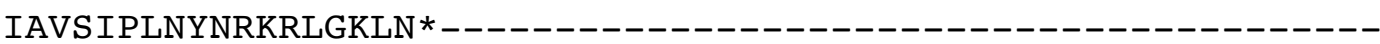

$* * * * * * * * * * * *::$ : : :
660

610

720

610

780

610

840

610

900

668

180

138 


\section{esr2a}

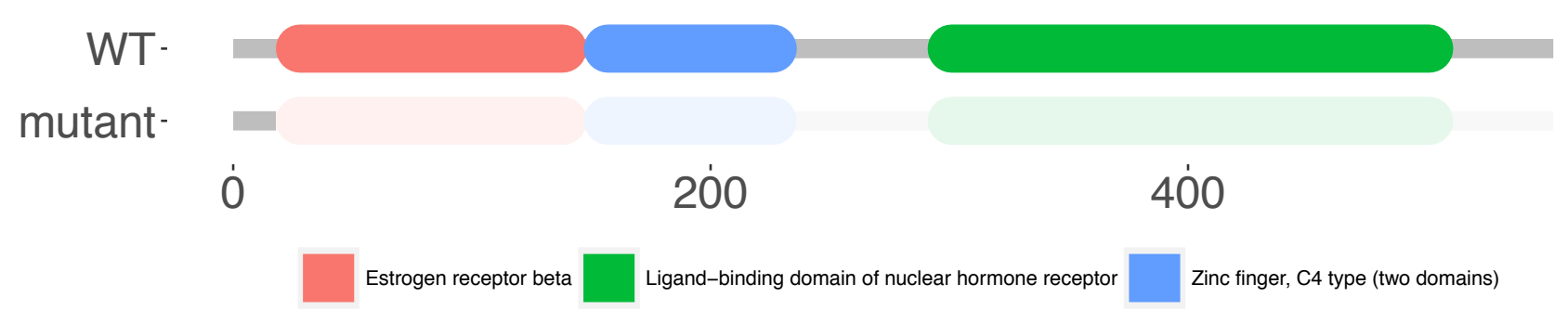

NM_180966.2

NV1182

caggaagtggactcaggccgtgtgggaggtcacatcctctccccc-atcttcaactcatc caggaagtggactcaggccgtgtgggaggtcacatcctctcccccaTtcttcaactcatc

NM_180966.2

NV1182
MSEYPEGDSPLLQLQEVDSGRVGGH ILSPIFNSSSP----SLPVENHPICIPSPYTDLGH MSEYPEGDSPLLQLQEVDSGRVGGH ILSPILQLILSISASGESPHLHPIALHRPWPRLQH $\star * * * * * * * * * * * * * * * * * * * * * * * * * * * * *::$ 


\section{gnrhr4}

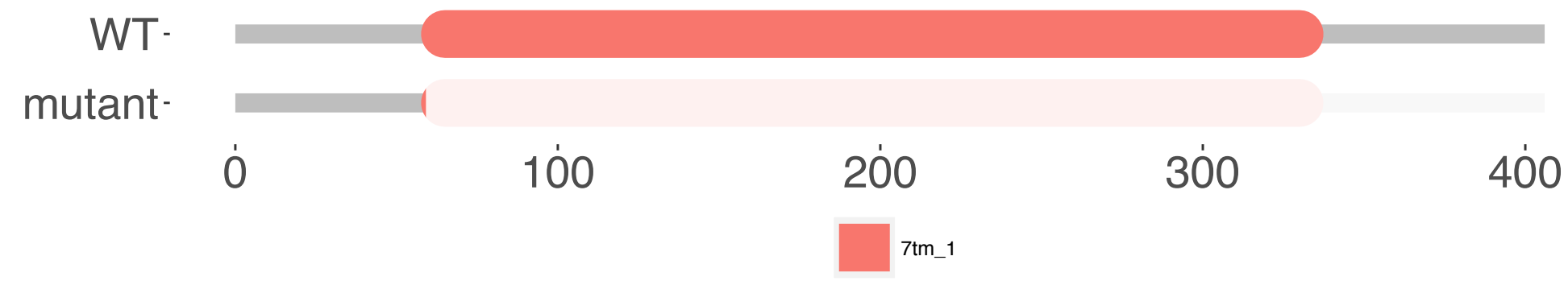

NM_001098193.1 NV 1280

NM 001098193.1 $\mathrm{NV} \overline{1} 280$

NM_001098193.1 NV 1280 agcaaaagtgagggtgataattaccttcactctttgcgcagtgtcagctgtgtgcaacct agcaaaagtgagggtgataattaccttcactctttgcgcagtgtcagctgtgtgcaacct $* * * * * * * * * * * * * * * * * * * * * * * * * * * * * * * * * * * * * * * * * * * * * * * * * * * * * * * * * * * *$ cggcg--tgttgtgggctgccagcaccaacaacaagcgcaagtcccatgtacgaatttta cggcAACGgttgtgggctgccagcaccaacaacaagcgcaagtcccatgtacgaatttta

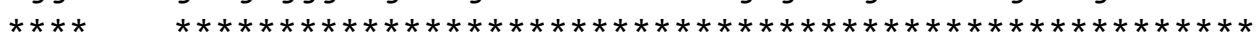

VSAVCNLGVLWAASTNNKRKSHVRILIMNLTVADLLVTF IVMPVDAAWNITVQWLAGDLA VSAVCNLGNGCGLPAPTTSASPMYEF *

$* * * * * * * *$ 


\section{grm5a}

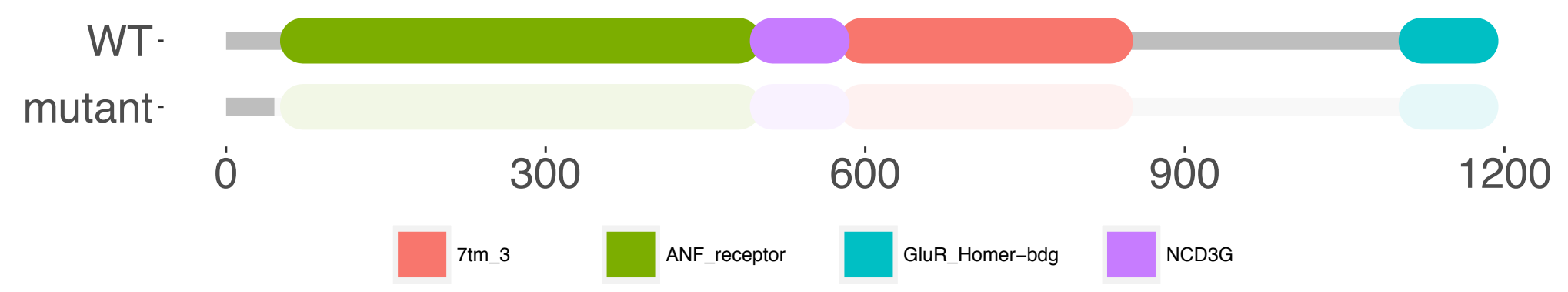

XM_009305305.2

caaggtccacgagcgcaagtgtggcgccgtccgagagcagtatggcatccagcgggtgga

360

NV1162 


\section{homer1b}

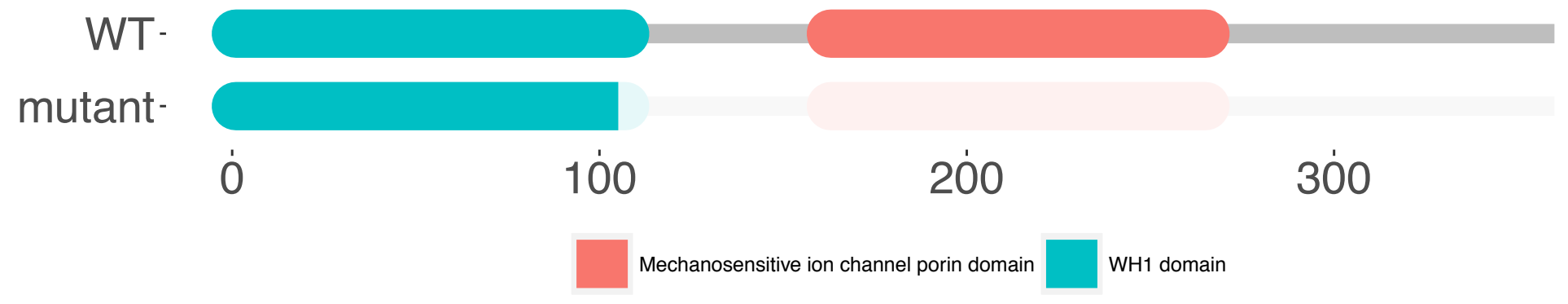

NM_001326290.1

NV1165

NM_001326290.1

NV1165 tttgcagagtacaaagaagcagccagaatagccaaggagaagtctctagaaaagatggag tttgcagagtacaaagaagcagccagaatac-caaggagaagtctctagaaaagatggag

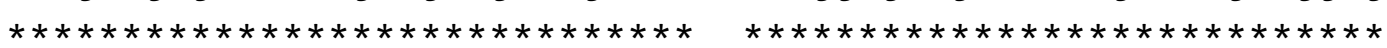

ITPNMSFTKTSQKFGQWADSRANTVYGLGF SSEHHLAKFADKFAEYKEAARIAKEKSLEK ITPNMSFTKTSQKFGQWADSRANTVYGLGFSSEHHLAKFADKFAEYKEAARIPRRSL *--

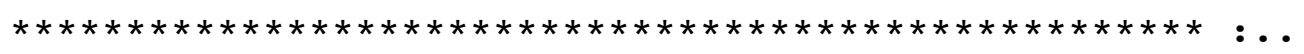




\section{immp2l}

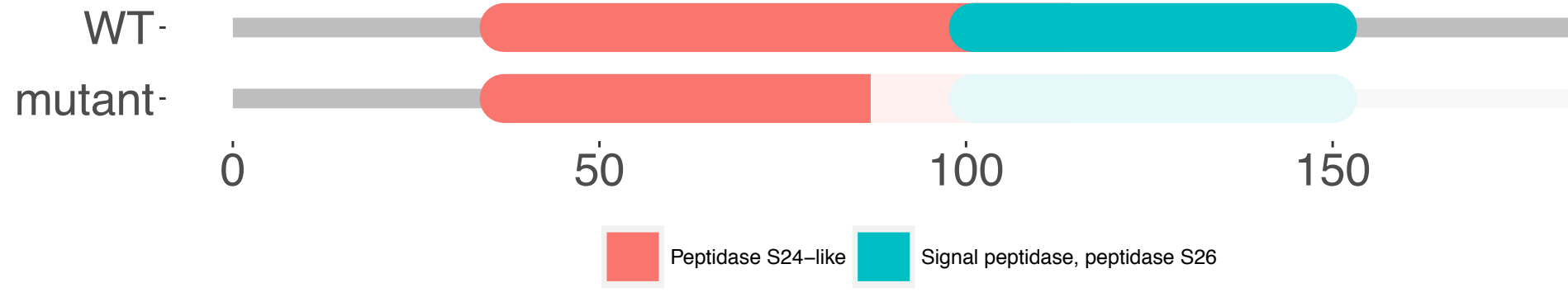

NM_001003755.2

NV1092

NM_001003755.2

NV1092 gatcatcaaacgggtcatcgggatcgaaggggacttcattaaaacgctgggatataaaaa gatcatcaaa-------tcgggatcgaaggggacttcattaaacgctgggatataaaa

NRWSVRNYHVQRGDIVSVLSPKNPQQKI IKRVIGIEGDF IKTLGYKNRYVRVPDGHLWIE NRWSVRNYHVQRGDIVSVLSPKNPQQKIIKSG--SKGTSLKRWDIKTVM*

120 


\section{kctd13}

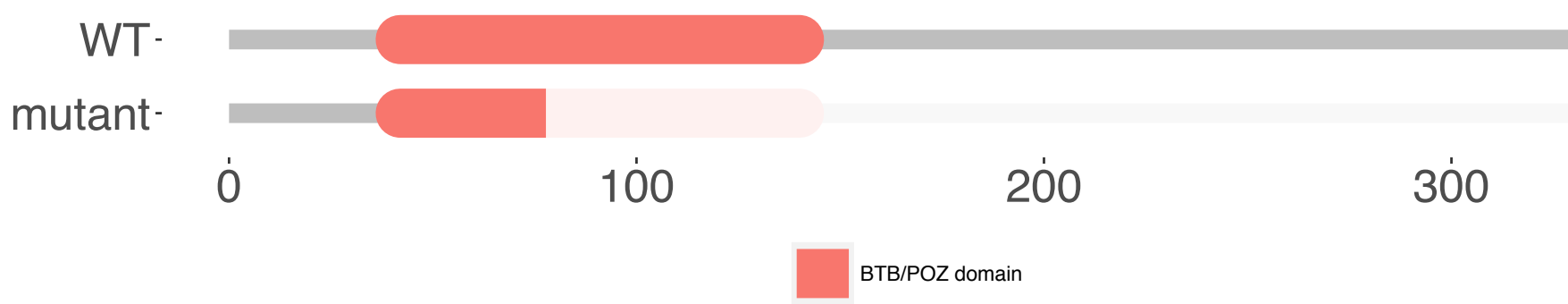

NM_001077151.1 NV966

NM_001077151.1 NV966

NM_001077151.1 NV966

NM 001077151.1 NV966 agcatcgactctgaaggctgggtggtgttggaccgatgcgggagacacttttccctggtg agcatcgactctgaaggctgggtggtgtt--------gcgggagacacttttccctggtg $* * * * * * * * * * * * * * * * * * * * * * * * * * * * *$ $* * * * * * * * * * * * * * * * * * * * * * *$

MSAEASGSSGGHAVTVSGSSPSSSSHVGDEKPGRSLVSSKYVKLNVGGTLHYTTVQTLSK MSAEASGSSGGHAVTVSGSSPSSSSHVGDEKPGRSLVSSKYVKLNVGGTLHYTTVOTLSK

$\star * * * * * * * * * * * * * * * * * * * * * * * * * * * * * * * * * * * * * * * * * * * * * * * * * * * * * * * * * * *$

EDSLLRSICDGSTEVSIDSEGWVVLDRCGRHFSLVLNFLRDGTVPLPDSTRELEEVLKEA EDSLLRSICDGSTEVSIDSEGWVVLRETLFPG----------VELPAGRD--------S

QYYRLQGLVQHCLSTLQKRRDVCRGCHIPMITSAKEEQRMIATCRKPVVKLQNNRGNNKY

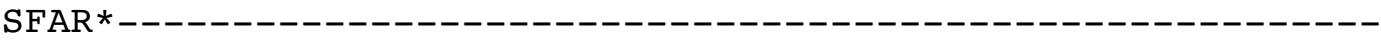
.: * 


\section{Irrn3}

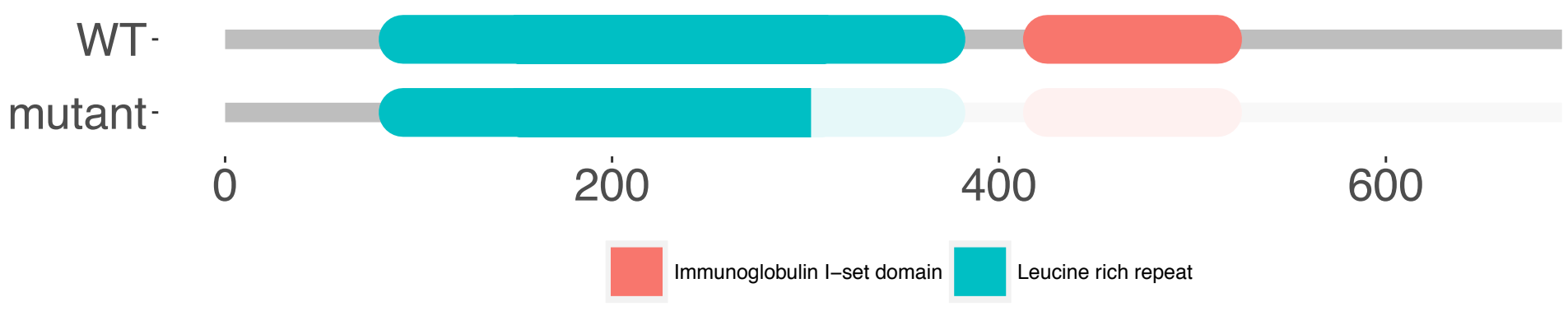

XM_005163071.3 NV971

XM_005163071.3 NV971 aagccacaaacaacccccgactttcctatattcacccaaatgctttttctcaactcccaa aagccacaa-----ccccgactttcctatattcacccaaatgctttttctcaactcccaa
DSFALHNLPELTKIEATNNPRLSYIHPNAFSQLPRLESLMLNSNALRALHHITVESLPNL DSFALHNLPELTKIEATTPTF-LY-SPKCFFSTPKTGVLDAE*--------------$* * * * * * * * * * * * * * * * *$. 


\section{nfkb1}

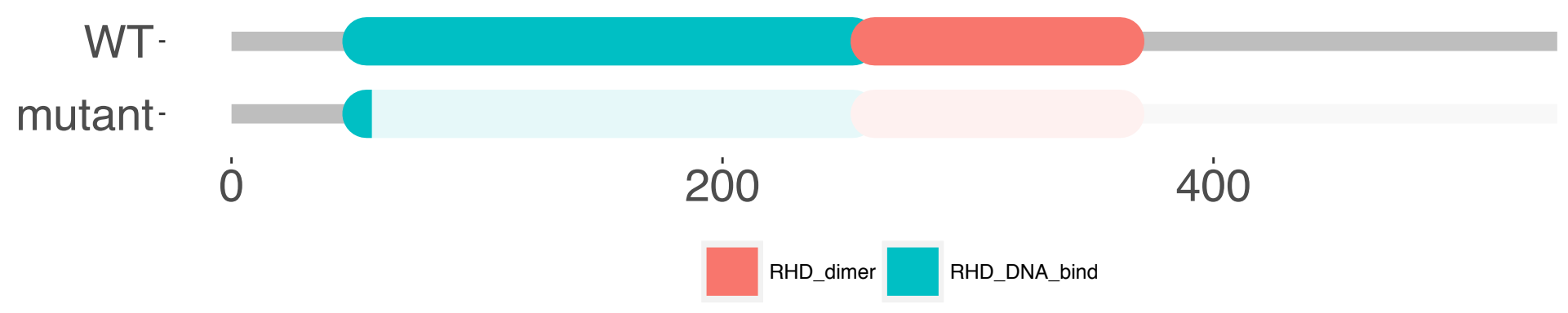

NM_001353873.1

NV1244

NM_001353873.1

NV1244

NM_001353873.1

NV1244

NM 001353873.1 NV1244
GCTTACAAATCACAGAACAACCAAAACAGAGGGGCTTCAGGTTCCGCTATGGGTGTGAGG GCTTACAAATCACAGAACAACCAAAACAGAGGGGCTTCAGGTTCCGTGTGAGGGCACGGA $* * * * * * * * * * * * * * * * * * * * * * * * * * * * * * * * * * * * * * * * * * * * * * * * *$ * GCCCGT-------CTCACGGAGGGCTTCCAGGGGCCTCCAGCGAGAAGAACCGCAAGT GGTGTGAGGTGTGAGGGCACGGAGGGCTTCCAGGGGCCTCC--_---_----AGCGAG *

$* * * * * * * * * * * * * * * * * * * * * * * *$

CCTACCCACAAGTGCAGATCTGTAATTATCAGGGCCCTGCTCGTGTGGTGGTCCAGCTGG AAGAACCACAAGTGCAGATCTGTAATTATCAGGGCCCTGCTCGTGTGGTGGTCCAGCTGG

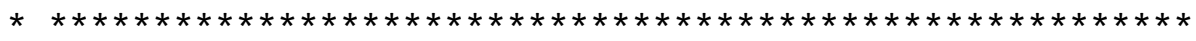

PKQRGFRFRYGCEGPSHGGLPGASSEKNRKSYPQVQICNYQGPARVVVQLVTNSQHPHLH 


\section{oxt}

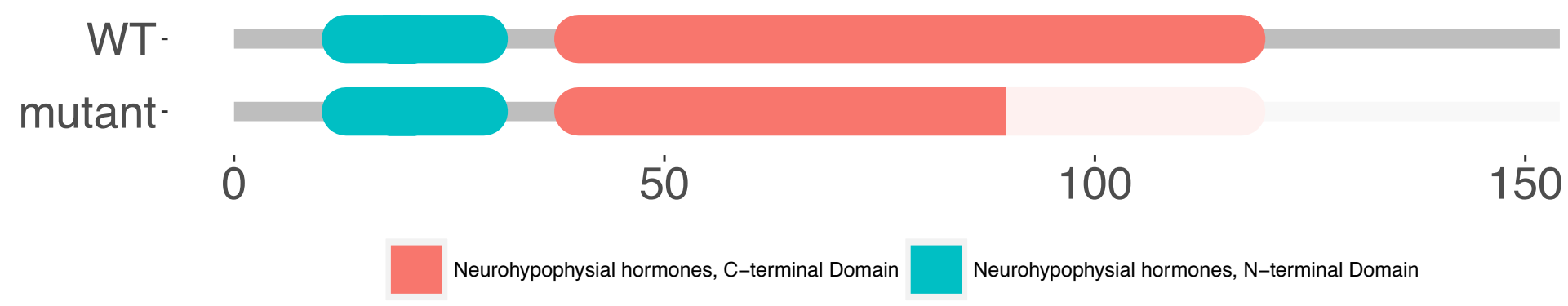

NM_178291.2 ccttctccgtgtgagatgtctggaaaggcetgcggttatgagggacgctgcgctgctcct

NM_178291.2

nv1081

GEGIGCLVGSPETLRCLEEDFLPSPCEMSGKACGYEGRCAAPGVCCDSEGCSVDQSCVDG 


\section{sapap2}

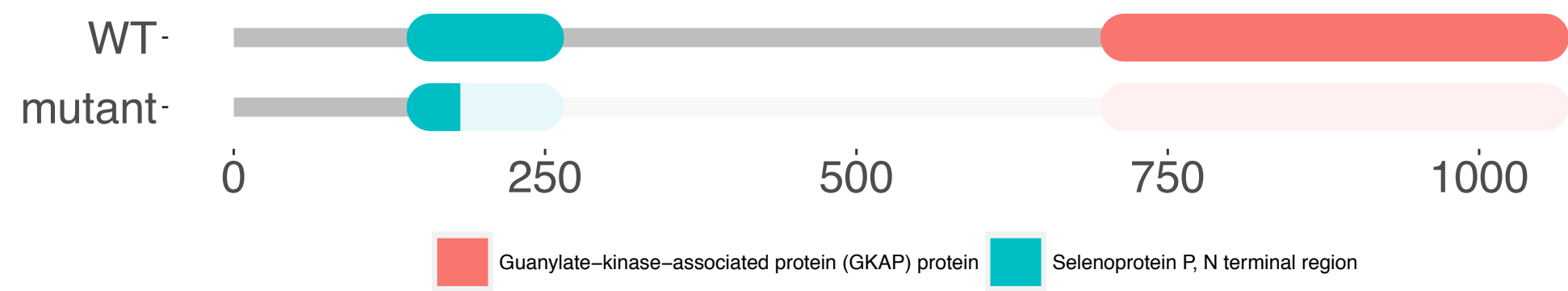

XM_680713.7

cttccaagatgaacggcaccaagggagactctcgctcagacagcggacaccaccaccatc 


\section{scn1lab}

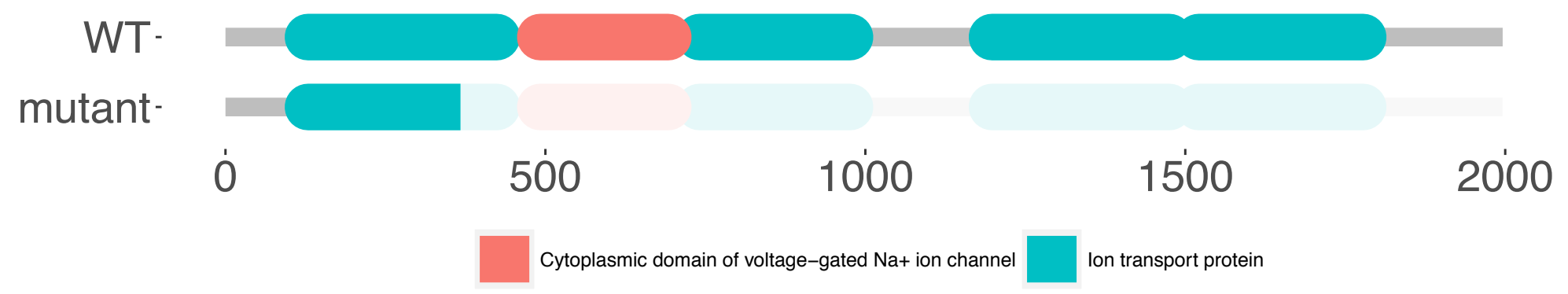

XM_005165770.3 NV978

tctatctggttaatctcatcctggctgtggtggccatggcgtatgatgagcagaaccagg 


\section{shank3b}

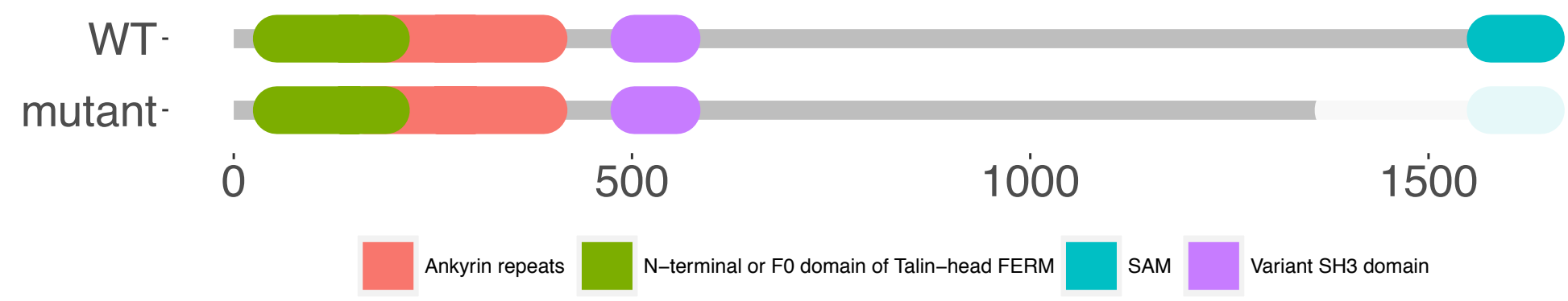

XM_009300184.2

NV1061

XM_009300184.2

NV1061 ttccaaactgtggggggaggagccccgggcccagatggggtcatcagatgaaagtcggcc ttccaaactgtgggg----------------------ggtcatcagatgaaagtcggcc

PRYLFQRRSKLWGEEPRAQMGSSDESRPAAMGAELLSKDTHSLGEEPPMGAPLDPGRRSP PRYLFQRRSKLWGVIR*

$\star * \star * * * * * * * * * *$
4440

4417 


\section{slc18a2}

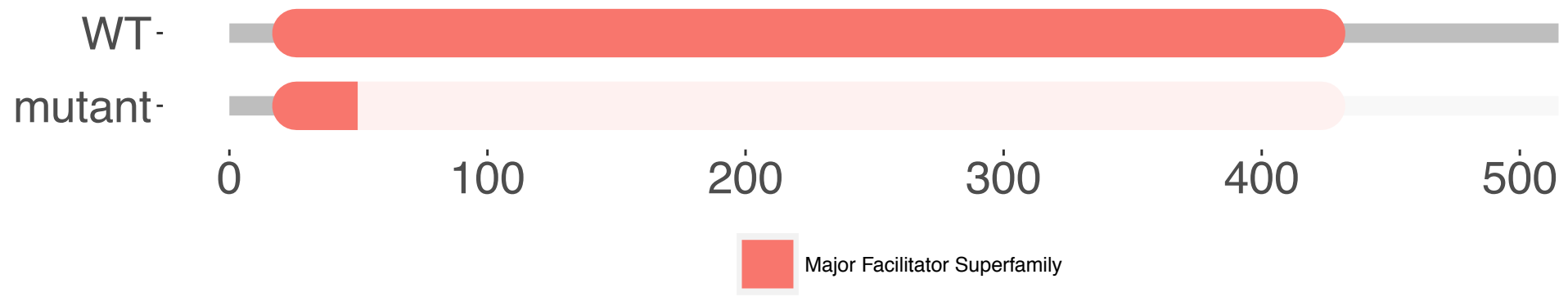

NM_001256225.2

NV1206

NM_001256225.2

NV1206

NM_001256225.2

NV1206

NM_001256225.2

NV1206

NM_001256225.2

NV1206

NM_001256225.2

NV1206 ggtggacgacgaggctgctcagatggttaagaatcactccatgacccctctttctccatc

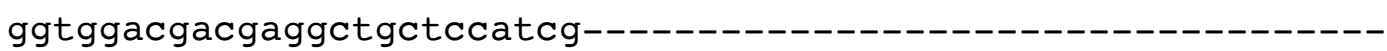
gagcacctttcagagcattgtgtctttgtatgacaacaccacacgtgtgacaggcttcag -agcacctttcagagcattgtgtctttgtatgacaacacc-------------------cccgcagatgagcacagctggtccaatgagcctggctcctacttttgtgagccctcaaa

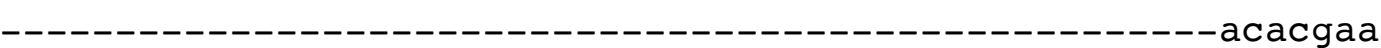
ccagtccgactgtcccaaagcagatgaccagcttctgaacgagaatgtgaaagtgggtct ccagtccgactgtcccaaagcagatgaccagcttctgaacgagaatgtgaaagtgggtct

MGLFDALRDFSLLTWLREERQSRRLILLIVF IALLLDNMLLTVVVPI IPSYLYTVDDEAA MGLFDALRDFSLLTWLREERQSRRLILLIVF IALLLDNMLLTVVVPIIPSYLYTVDDEAA $* * * * * * * * * * * * * * * * * * * * * * * * * * * * * * * * * * * * * * * * * * * * * * * * * * * * * * * * * * * *$ QMVKNHSMTPLSPSSTFQSIVSLYDNTTRVTGFSPQMSTAGPMSLAPTFVSPQNQSDCPK ----------PSSTFQS IVSLYDNTTRTSPTVPKQMTSF * 


\section{slc1a1}

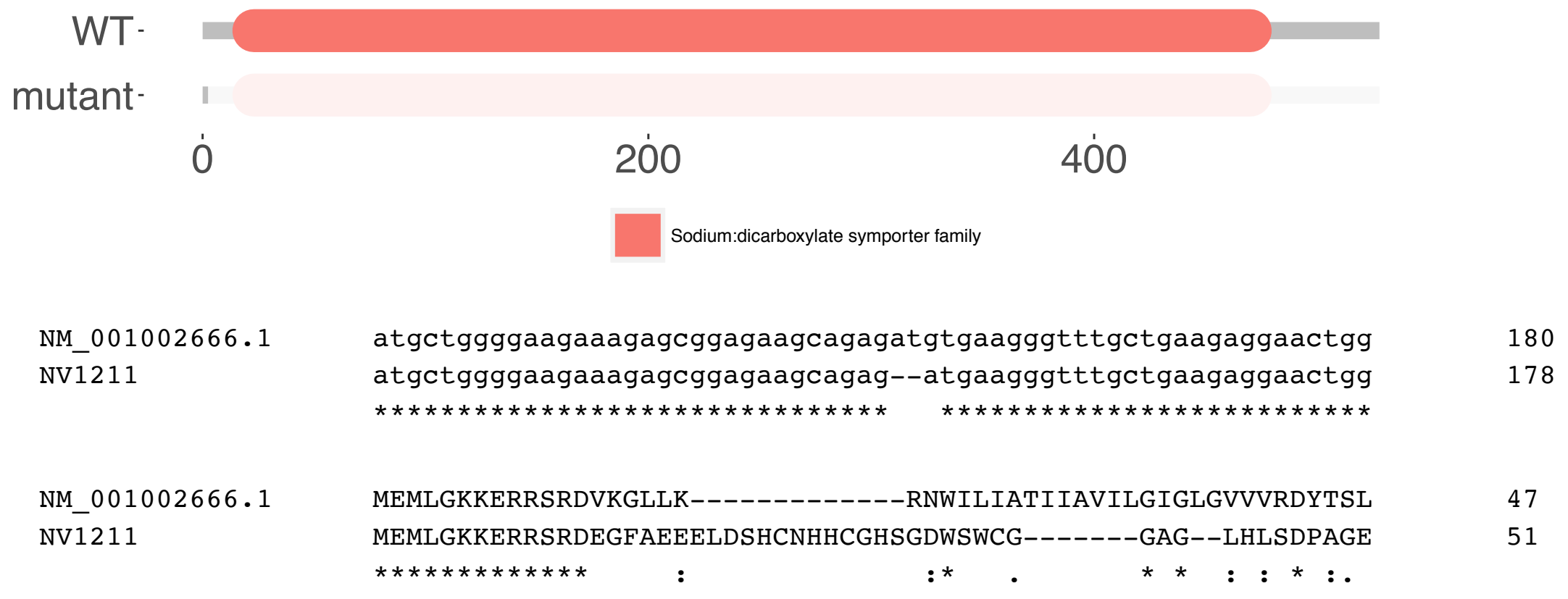




\section{slc22a15}

WT-

mutant-

ó

100

200

300

400

500

Major Facilitator Superfamily

NM_001109699.1

tctggaatggatttagaggaggctttccaggtTGGAGGATGTTCCAGGATGTTCTCTCTA

152

NV1209

NM_001109699.1

---ggttggagagttcggaagtcaccagaagcgaatgatcacagtgctggtgttcctcca

180

TCAggttggagagttcggaagtcaccagaagcgaatgatcacagtgctggtgttcctcca

209

NV1209

240

NM_001109699.1

MDLEEAFQVVGEFGSHQKRMITVLVFLQIYMACQSMLI ILVGAVPEYHIEPVTEGVDGDV

NV1209 


\section{slc25a27}

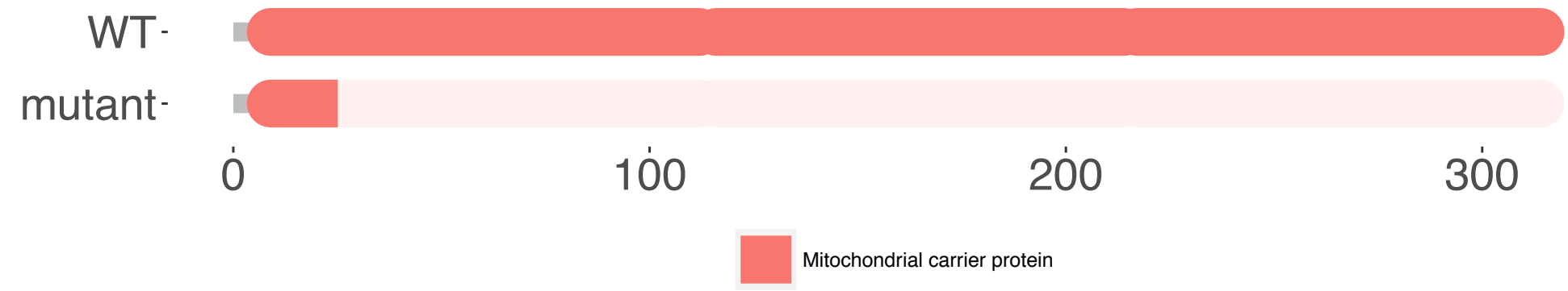

NM_200341.1

cagaattggtcacattccccctggatcttaccaaaaccagactccagatccagggtgaag

NM_200341.1 $* * * * * * * * * * * * * * * *$

gcagatctggaaagaatggtggaagcgtacagactcagaaatacaggggcatgttgagca 


\section{slc39a11}

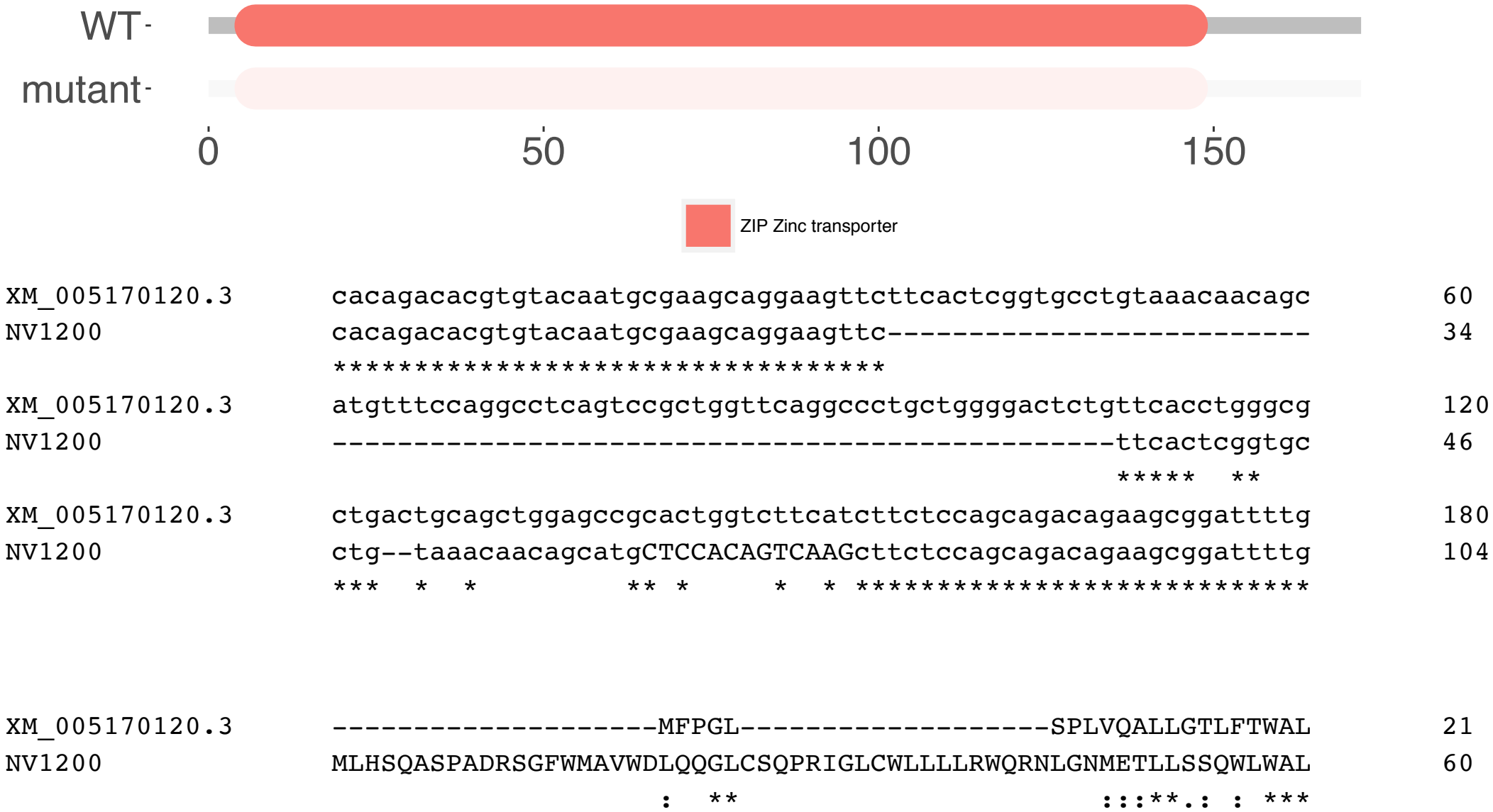




\section{slc6a3}

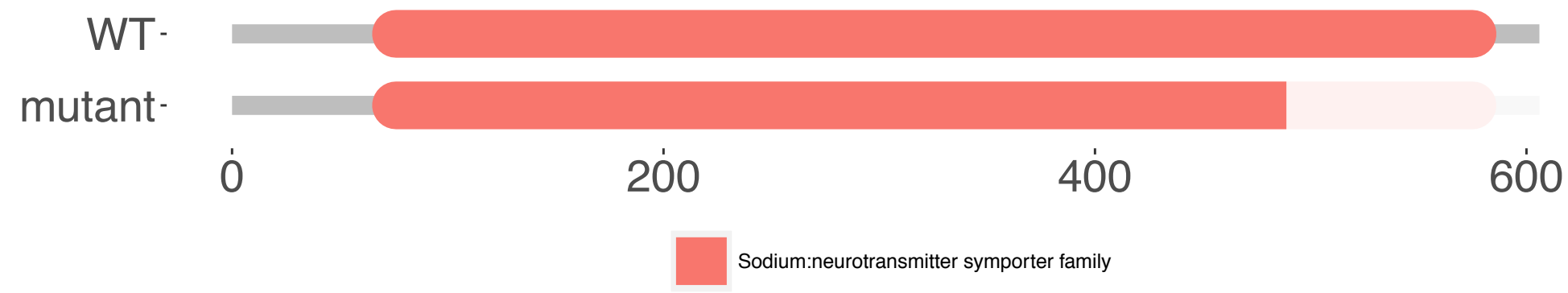

NM_131755.1

NV1087

NM_131755.1

NV1087

NM 131755.1

NV1087

tttacgctgttggaccacttcgctgcggggacgtcaattctctttggagtactaattgag tttacgctgttggaccacttcgctgcggggacgtcaattctctttggagtactaattgag $* * * * * * * * * * * * * * * * * * * * * * * * * * * * * * * * * * * * * * * * * * * * * * * * * * * * * * * * * * * *$ gccatcggcatcgcctggttttacggagtggatcgcttcagtgatgatatcgaggagatg g-------catcgcctggttttacggagtggatcgcttcagtgatgatatcgaggagatg

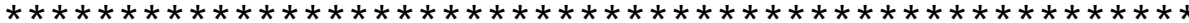

FTLLDHFAAGTSILFGVLIEAIGI--AWFYGVDRFSDDIEEMIGQRPGLYWRLCWKFVSP FTLLDHFAAGTSILFGVLIEASPGFTEWIASV MISRR* 538

1500 


\section{slc6a4a}

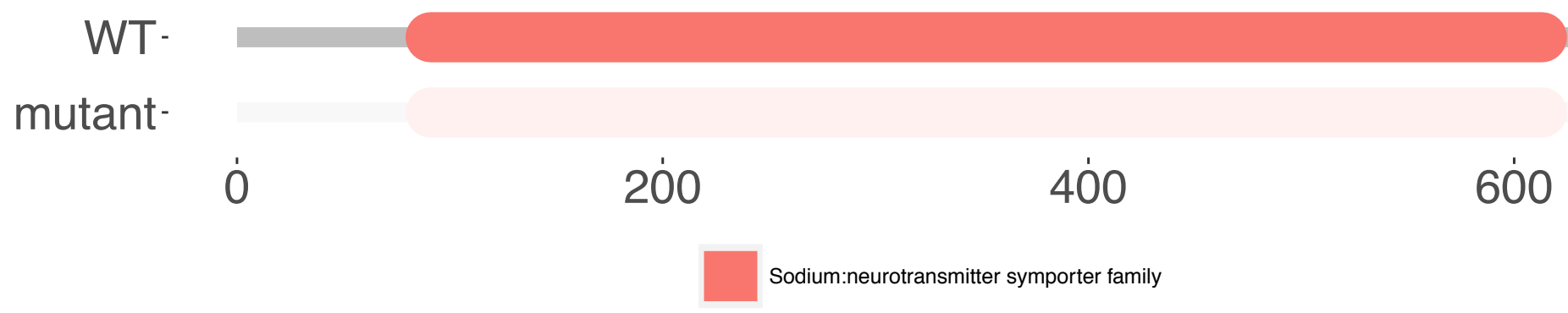

XM_009291669.2

aagagtacgggggagagcagcagaaagtgccggagtctcaagagaacggcaggctggttg

NV1208

aagag----ggggagagcagcagaaagtgccggagtctcaagagaacggcaggctggttg

836

XM_009291669.2

MDMKESMMMNQEYGGEQQKVPESQENGRLVVDSVPEKDQKSGSGPGQVSNGYRSTSPQSP 


\section{slc6a8}

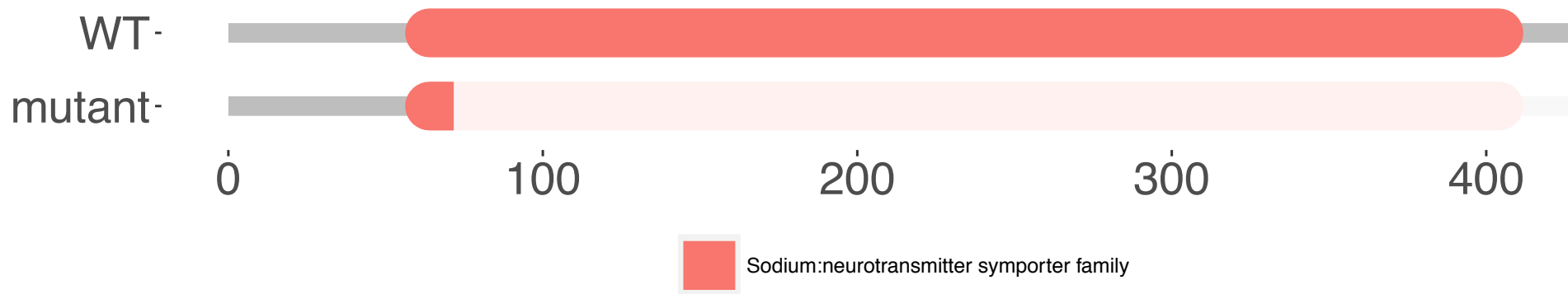

XM_005166721.2

NV1197

XM_005166721.2

NV1197

XM_005166721.2

NV1197

XM_005166721.2

NV1197

XM_005166721.2

NV1197

XM_005166721.2

NV1197 aatggaaacgcgcacccaagcgtcaacgggaccctcaacggggggcccatgtcagcggct aatggaaacgcgcacccaagcgtcaacgggaccctcaTGTCggggcccatgtcagcggct

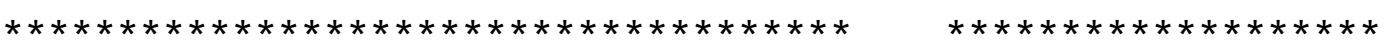
accggagccatctcggcggtggagaagaagagggagggctaccccgagcgagagacgtgg accggagccatctcggcggtggagaagaagagggagggctaccccgagcgagagacgtgg

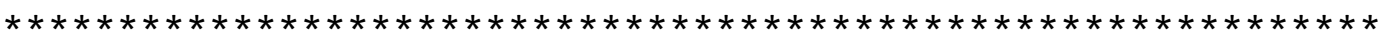
accagacagatggacttcatcatgtcctgcgtgggttttgcagtggggctggggaacgtc accagacagatggacttcatcatgtcctgcgtgggttttt-----------ggggaacgtc

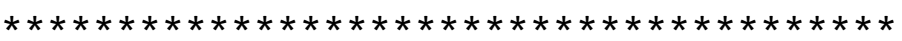
$\star * * * * * * * * *$

MEKSSLDADCCALNMVLVEEKKGHLIPNGNAHPSVNGTLNGGPMSAATGAISAVEKKREG MEKSSLDADCCALNMVLVEEKKGHLIPNGNAHPSVNGTLMSGPMSAATGAISAVEKKREG

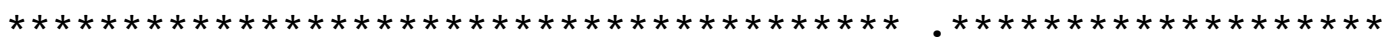
YPERETWTRQMDF IMSCVGFAVGLGNVWRFPYLCYKNGGGVFLIPYVLF IFLGGIPIFFL YPERETWTROMDF IMSCVGFWGTSGASRTCATKTVEE-SSSFPMCYSYFW----------

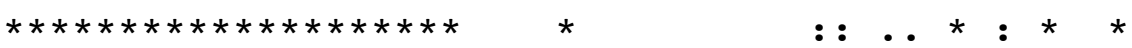
EIALGQFMKAGS INVWNIAPLFKGLGYASMVIVFFCNTYY IMVLAWGFYYFIKSFNATLP

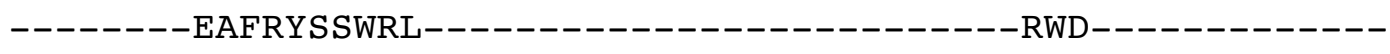
:* .*. : *. 
srr

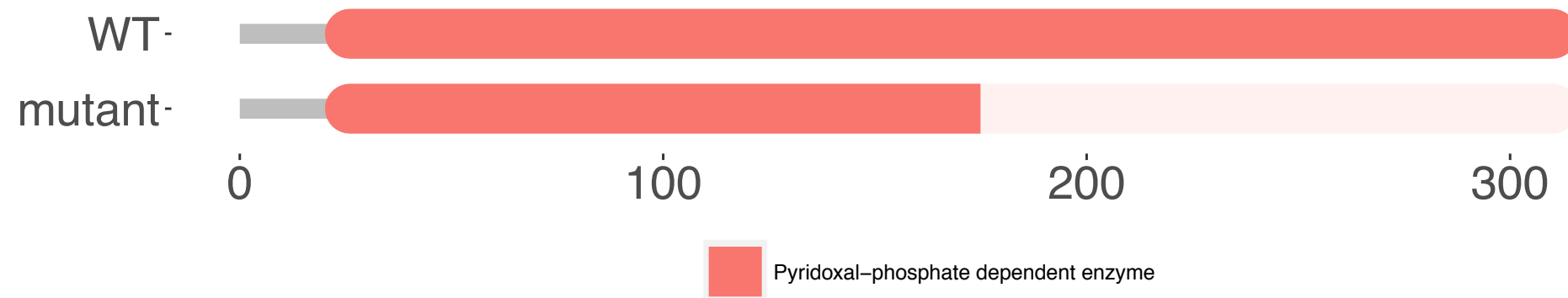

XM_002661465.4 NV1157

XM_002661465.4 NV1157

XM_002661465.4 NV1157

XM_002661465.4 NV1157 gacattccagcttaggttttgagatcctggatgtcgtaccctatccagatgtggtggttg gacattccagcttaggtttgagatcctggatgtcgtaccctatccagatgtggtggttg $\star * * * * * * * * * * * * * * * * * * * * * * * * * * * * * * * * * * * * * * * * * * * * * * * * * * * * * * * * * *$ tttgctgtggtggtggagggttactttctggtgtggccgctgccatcaaactgtctggtt t-------------------tactttctggtgtggccgctgccatcaaactgtctggtt *

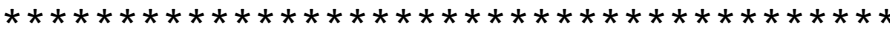

NLGVEVERVPTTOLMGVVNRCVQEDGMTFLHS IDDPDLIAGHSSLGFEILDVVPYPDVVV NLGVEVERVPTTQLMGVVNRCVQEDGMTFLHS IDDPDLIAGHSSLGFE ILDVVPYPDVVV

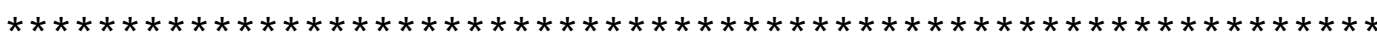
VCCGGGGLLSGVAAAIKLSGCEDTKIYGVEPEGACTMYKSF IEKRPVGMDAKS IASGLAP VTFWCGRCHQTVWL *-_-_-_-_-_-_-_-_-_-_-_-_-_-_-_-_-_-_-_-_-_-_-_-_
660

660

720

700

180

180

240

194 


\section{tph2}

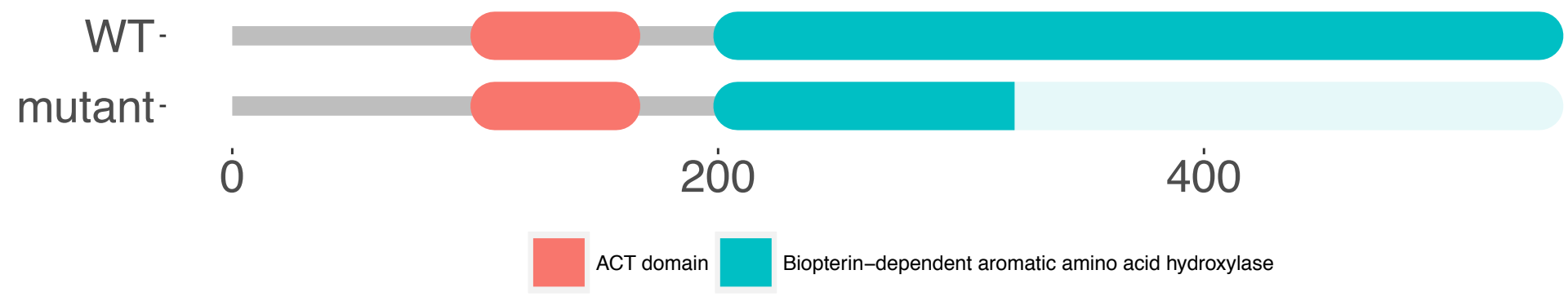

NM_001310068.1 NV1089

NM_001310068.1 NV1089 aaggcctgtggctggatatctctcaccacgagacttcctggctggactggcttatcgagt aaggcctgt-gctggatatctctcaccacgagacttcctggctggactggcttatcgagt

LLTKHCGYREDNIPQLEDVSLFLRERSGFTVRPVAGYLSPRDFLAG-LAYRVFNCTQYIR LLTKHCGYREDNIPQLEDVSLFLRERSGFTVRPVLDISHHETSWLDWLIECLIALSIYVT $* * * * * * * * * * * * * * * * * * * * * * * * * * * * * * * * * *$
1140

1139

359

360 


\section{ube3a}

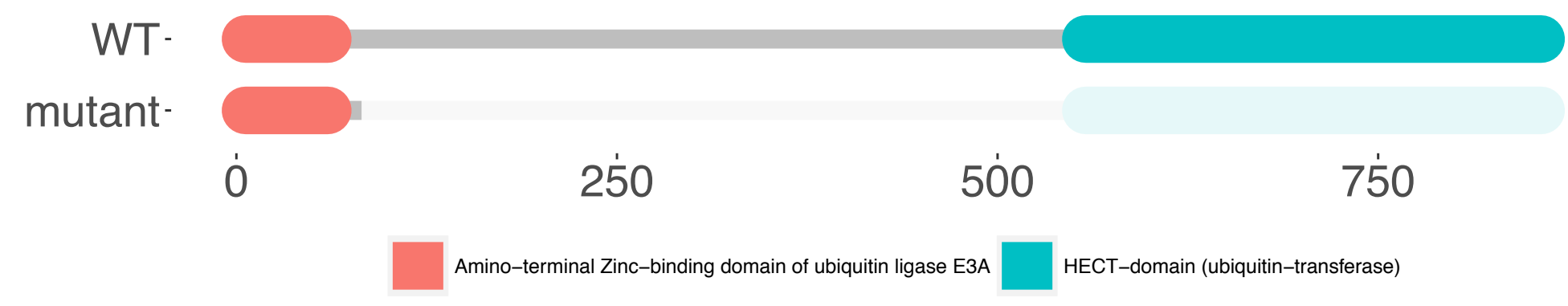

NM_001007318.1

ggacctgccacccacaagggaggactttagagatctgaatttcttgactgaggataaggt 
Suppigemen Supplemental Fertiffed by peer review) is the author/funder. All rights reserved. No reuse allowed without permission.

\begin{tabular}{|c|c|c|c|}
\hline Unpolarized & Kullback-Leibler divergence & Polarized & Kullback-Leibler divergence \\
\hline 'immp2IHO' & Inf & 'immp2IHO' & Inf \\
\hline slc18a2HT' & 0.503872588 & 'adra1aaHO' & 0.408321982 \\
\hline 'scn1abHT' & 0.475243162 & 'slc25a27HO' & 0.401431298 \\
\hline slc6a4aHO' & 0.450082277 & 'drd4-rsHO' & 0.380778006 \\
\hline 'drd4-rsHO' & 0.372634411 & 'chrna2aHO' & 0.352634968 \\
\hline 'disc1KO' & 0.361275622 & 'slc18a2HT' & 0.335771101 \\
\hline 'drd4aHO' & 0.360377693 & 'disc1KO' & 0.317098761 \\
\hline 'slc1a1HO' & 0.344640154 & 'homer1bHO' & 0.307501307 \\
\hline 'slc6a8HO' & 0.332613849 & 'slc6a8HO' & 0.242433203 \\
\hline 'homer1bHO' & 0.328809872 & 'drd4aHO' & 0.232985499 \\
\hline 'chrna2aHO' & 0.256689064 & 'scn1abHT' & 0.229660386 \\
\hline 'chrm4aHO' & 0.254995398 & 'gnrhr4HO' & 0.229206427 \\
\hline 'gnrhr4HO' & 0.249192827 & 'oxtKO' & 0.170716837 \\
\hline 'adra1aaHO' & 0.245908343 & 'drd2bKO' & 0.165339327 \\
\hline 'slc6a3KO' & 0.239132287 & 'slc6a4aHO' & 0.135011208 \\
\hline 'adra1abHO' & 0.22832634 & 'slc1a1HO' & 0.124852827 \\
\hline 'drd2bKO' & 0.223290031 & 'esr2aHO' & 0.115764118 \\
\hline 'sapap2KO' & 0.215181308 & 'chrm4aHO' & 0.115151629 \\
\hline 'esr2aHO' & 0.2130425 & 'srrHO' & 0.099703579 \\
\hline 'dlg4aHO' & 0.210317791 & 'dlg4aHO' & 0.097755979 \\
\hline 'chd8KO_WT' & 0.189007333 & 'drd3HO' & 0.088199216 \\
\hline 'tph2HO' & 0.188094395 & 'slc22a15HO' & 0.079772629 \\
\hline 'srrHO' & 0.186947659 & 'slc39a11HO' & 0.070391332 \\
\hline 'slc25a27HO' & 0.185835058 & 'KCTD13KO' & 0.065474473 \\
\hline 'nfkb1HO' & 0.180801278 & 'sapap2KO' & 0.064423091 \\
\hline 'oxtKO' & 0.177612315 & 'drd1bHO' & 0.053110873 \\
\hline 'shank3bKO' & 0.16671958 & 'Irrn3KO' & 0.052257913 \\
\hline 'drd3HO' & 0.161913606 & 'adra1abHO' & 0.045964875 \\
\hline 'drd1bHO' & 0.099974442 & 'shank3bKO' & 0.045234588 \\
\hline 'slc22a15HO' & 0.049265198 & 'nfkb1HO' & 0.035939197 \\
\hline 'grm5aHO' & 0.035304671 & 'slc6a3KO' & 0.031863257 \\
\hline 'slc39a11HO' & 0.030706812 & 'tph2HO' & 0.030858559 \\
\hline 'KCTD13KO' & 0.021098744 & 'grm5aHO' & 0.018200187 \\
\hline 'ube3aKO' & 0.017664977 & 'ube3aKO' & 0.006102855 \\
\hline 'Irrn3KO' & 0.013683683 & 'chd8KO_WT' & 0.00384635 \\
\hline 'WT' & 0 & 'WT' & 0 \\
\hline
\end{tabular}

